\title{
1 Vertical Deformation and Residual Altimeter Systematic Errors \\ around Continental Australia Inferred from a Kalman-Based Approach
}

\author{
Mohammad-Hadi Rezvani ${ }^{1}$, Christopher S. Watson ${ }^{1}$, Matt A. King ${ }^{1}$ \\ ORCID IDs: 0000-0001-9754-2196, 0000-0002-7464-4592, 0000-0001-5611-9498 \\ ${ }^{1}$ School of Geography, Planning, and Spatial Sciences, University of Tasmania, Hobart, Tasmania, \\ Australia
}

Corresponding author: Mohammad-Hadi Rezvani (mohammadhadi.rezvani@utas.edu.au)

This is a non-peer reviewed preprint submitted to EarthArXiv. This preprint has also been submitted to the Journal of Geodesy for peer review.

Key Points:

- We developed a new method to estimate time-variability of vertical land motion and altimeter systematic errors, tested around Australia.

- Our approach confirms widespread subsidence across the Australian region, and this is not explained by glacial isostatic adjustment alone.

- We identify noise driven by likely residual oceanographic signals between coastal and offshore locations as a hard limit to the ability to resolve time-variable signals.

- Averaged rate of absolute sea-level rise at TGs is higher than previously published estimates suggesting an acceleration in sea-level. 


\section{Abstract}

We further developed a space-time Kalman approach to estimate time-variable signals in residual altimeter systematic errors and vertical land motion (VLM) around the Australian coast since the 1990s, through combining multi-mission absolute sea-level (ASL), relative sea-level (RSL) from tide gauges (TGs) and GPS heights records. Our results confirmed continent-wide subsidence and TG-specific VLMs yielding a $\sim 40 \%$ reduction in RMSE of geographical ASL variability, compared with rates determined using spatially interpolated GPS velocities that fail to capture localized trends by up to $\sim 1.5 \mathrm{~mm} / \mathrm{yr}$. Stacked time series of non-linear deformation at TGs and nearby GPS showed some correlation, suggesting the technique was partially successful in reflecting the surface loading. Site-by-site inspection revealed spurious non-linearity likely caused by residual oceanographic signals present between the TG and altimeter measurement locations. Our average mission-specific error estimates are small but significant, typically within $\sim \pm 0.5-1.0$ $\mathrm{mm} / \mathrm{yr}$, with negligible effect implied on the overall rate of ASL. Analysis of the time variability of altimeter errors confirmed stability for most missions except for Jason-2 with an anomaly reaching $\sim 2.8 \mathrm{~mm} / \mathrm{yr}$ in the first $\sim 3.5$ years of operation which is supported by analysis from the Bass Strait altimeter validation facility. Weak correlation with the dominant climate mode suggests potential deficiencies in the resolution of the time-variable gravity field used for orbit determination as a possible cause, yet other drivers cannot be discounted. Our approach advances the ability to estimate TG-specific VLMs and regional altimeter systematic errors, and highlights that residual oceanographic signals remain a fundamental limitation to such techniques.

Keywords: Vertical land motion; Altimeter systematic errors; Australian region; Sea-level rise

\section{Introduction}

Vertical land motion (VLM) of the Earth's surface is a key link between changes in absolute sea-level (ASL) derived in a geocentric frame from satellite altimeters (ALTs) and relative sealevel (RSL) from tide gauges (TGs) attached to the crust in a local reference frame (e.g., White et al., 2014). VLM is often assumed to be dominated by glacial isostatic adjustment (GIA, e.g., Peltier et al., 2018), yet tectonics (e.g., Bevis \& Brown, 2014) and climate-induced mass redistribution of 
the atmosphere, ocean, and continental waters (e.g., Santamaría-Gómez \& Mémin, 2015) play a noticeable role in driving VLM over various timescales. Anthropogenic effects also contribute at local scales (e.g., Dangendorf et al., 2015; Raucoules et al., 2013). Quantifying VLM and its possible variability in time is required to improve our understanding of regional patterns of sealevel rise at the coast, and thus better planning of adaption strategies.

The Global Positioning System (GPS) has emerged as a main tool used to quantify VLM at TG locations (e.g., Hamlington et al., 2016; King et al., 2012; Santamaría-Gómez et al., 2012; Wöppelmann et al., 2009). As most TGs are not yet equipped with co-located or nearby GPS sites, and ellipsoidal height series are often short in comparison with the altimeter records (Bouin \& Wöpplemann, 2010), approaches seeking to estimate VLM from the combination of ALT and TG measurements have been explored (e.g., Wöppelmann \& Marcos, 2016). These alternate studies have provided new insight into VLM along the coasts yet have mostly neglected to evaluate residual systematic errors or bias drifts in the altimeter-specific datasets (Rezvani et al., 2021). The regional or global expression of these small errors (each within mission specifications expressed as global metrics) accumulated from individual systematic error components present in orbits, ranges, and other environmental and geophysical corrections (e.g., Fu \& Haines, 2013), thus requires further investigation. Regional differences in the leading orbit products alone can typically reach $\sim 2-4 \mathrm{~mm} / \mathrm{yr}$ over a typical mission lifespan (e.g., Couhert et al., 2015; Fu \& Haines, 2013), highlighting a major challenge in the attempts to derive local ASL or VLM with sub-mm/yr accuracy using altimetry products. Previous studies have also highlighted that the difference in ocean processes acting between the offshore altimetry and coastal TG locations may cause a substantial error in resulting estimates of VLM (e.g., Nerem \& Mitchum, 2002; Watson et al., 2015). The extent to which residual oceanographic variability (between sample locations of TG and ALT records) becomes a hard or fixed limit to the utility of the technique remains a vexing issue yet to be fully explored.

GPS observations of VLM across Australia suggest the continent is subsiding overall (Hammond et al., 2021; King et al., 2012; Riddell et al., 2020). Recent work by Riddell et al. (2020) using GPS time series showed the widespread pattern of subsidence cannot be fully explained by GIA. Earlier work also suggested subtle subsidence from spatially interpolating linear GPS rates to many Australian TGs (e.g., Burgette et al., 2013; White et al., 2014). This linear-only 
assumption was further challenged by Watson et al. (2010) who investigated anomalous subsidence on the Australian plate margin in response to the $2004 \mathrm{Mw} 8.1$ earthquake north of Macquarie Island. Riddell et al. (2021) subsequently pointed out that some regions of continental Australia are subject to small post-seismic relaxation which may express in the vertical component. Other known drivers of non-linearity include hydrological loads across the continent (e.g., Han, 2017), and more localized anthropogenic effects, such as across the Perth basin (e.g., Featherstone et al., 2015).

Estimation of VLM using ALT minus TG records around Australia has received some attention. Wöppelmann and Marcos (2016) used different gridded products (i.e., AVISO, CCI, CSIRO and GSFC) and pointed out that the ALT and TG combinations are likely to estimate reliable VLM in the Western Australia. Pfeffer et al. (2016) derived VLMs by differencing TG records and gridded SSALTO/DUACS ALT data and suggested a discrepancy with GIA predictions possibly due to changes in surface mass loading. More recently, Watson (2020) confirmed the general pattern of subtle subsidence, from differences taken between the SSALTO/DUACS grid points and TG observations. These approaches adopted gridded and not along-track products as well as a linear assumption for VLM and did not consider the residual mission-specific systematic errors over the region. Two key questions emerged when seeking to improve our understanding of VLM around the Australian coast using this technique. First, could the ALT-TG approaches be further developed to investigate potential time-variable signals in VLM and regional mission-specific systematic errors? Second, to what extent can the inclusion of multi-mission along-track altimeter data improve spatial sampling to mitigate the limitation driven by potential differential oceanographic signals between TG and ALT locations?

Here we address these questions by advancing the method set out by Rezvani et al. (2021) who applied an early version of the framework to the Baltic Sea region using altimetry records from reference-missions alone, along with a linear-only assumption for both the VLM and bias drift quantities. We further developed the space-time Kalman filtering and smoothing approach and applied it to examine the vertical stability of the Australian coast since the 1990s. We considered multi-mission datasets and simultaneously estimated the time-fixed and time-variable components of location-specific VLMs and residual altimeter-specific systematic errors in a regional context. 
We integrated measurements of ALT minus TG, ALT tandem/dual crossovers, and GPS bedrock heights, accounting for correlated noise and observational covariances across time and space.

To overcome the singularity of the underlying problem, we developed a refined multi-stage solution strategy to gradually estimate the highly correlated unknowns. We first improved the VLM trends at geodetic sites using linear estimates of altimeter bias drift computed from spatially interpolated GPS velocities. We subsequently explored our ability to simultaneously separate nonlinear evolution in mission-specific bias drifts from temporal variability in site-specific VLMs. We further investigated the agreement and spatial coherence of resultant ASL estimates at the TG and ALT measurement locations. Our multi-mission solution was also compared to the referencemission-only implementation to assess the benefit of the expanded constellation and improved spatial sampling over the continental shelf in this technique.

In the next section, we describe the main characteristics of our Kalman-based approach. We then present the key results from applying the method around the Australian continent. We refer the reader to supplemental information for a suite of sensitivity tests summarised in the subsequent section. We conclude with a discussion to highlight the strengths and inherent limitations of the approach.

\section{Datasets}

Our altimeter datasets include $1-\mathrm{Hz}$ along-track ASL from the reference missions (TOPEX, Jason-1, OSTM/Jason-2, and Jason-3 with temporal sampling every $\sim 9.9$ days) and to improve spatial sampling, the non-reference missions (ERS-2, Envisat, and SARAL/AltiKa with 35-day, and Sentinel-3A with 27-day, repeat sampling). All missions used orbit products computed relative to ITRF2008 (Altamimi et al., 2011). Altimeter datasets were retrieved from the Radar Altimeter Database System (RADS, Scharroo et al., 2013; data accessed March 31, 2020) and sampled at offshore Comparison Points (CPs) spaced by $20 \mathrm{~km}$, spanning from September 1992 to February 2020. The so-called cal-1 mode correction was not applied to the TOPEX data (Beckley et al., 2017). Following Watson et al. (2015), TOPEX-side A and -side B were treated as two different missions. We applied the solid-Earth tides and then removed the ocean tides and loadings using the FES2014 model (Lyard et al., 2021). We used the AVISO dynamic atmosphere corrections 
(DACs, https://www.aviso. altimetry.fr/) for ALT-TG, substituted with the MOG2D model for ALT crossover series. We applied the pole tides to the crossovers, but only the radial body pole tides to the ALT-TG combinations (e.g., Desai et al., 2015). Table S1 lists other geophysical and environmental corrections applied. We derived a priori estimates of ASL slope at the multi-mission CPs from the DTU15 mean sea surface as a function of the zonal-track distances (as per Rezvani et al., 2021).

We used hourly RSL series from a national network of 23 TGs accessible from the Australian Baseline Sea Level Monitoring Project (ABSLMP, and its Supplementary Stations) operated by the Australian Bureau of Meteorology (http://www.bom.gov.au/oceanography/projects/abslmp/ abslmp.shtml), with the timespan between January 1990 and February 2020. We limited the TG set to these gauges given they were sited in areas generally considered well connected to the open ocean thus minimising the potential for residual trend in the difference in ASL observed at the TG and suitably close yet offshore ALT measurement locations. We refer to Table S4 for the gauge specifications. We similarly applied the AVISO DACs to the RSL records to account for the impact of atmospheric pressures on the sea-level variability (White et al., 2014). We then estimated and removed the ocean tides from the RSL records using the UTide software (Codiga, 2011), considering nodal modulations and the same constituents as those used in the FES2014b model as used for the ASL time series.

Daily height series were used from 210 GPS sites provided by the Nevada Geodetic Laboratory (NGL, Blewitt et al., 2018; data accessed February 26, 2020). These data are relative to the ITRF2008 and have a maximum span of January 1994 to September 2019 (noting that from 1994 to 2009 the site distribution is sparse given network densification occurred over 2009 to 2014 ). The spans of individual GPS series are listed in Table S5.

For comparison purposes, we linearly interpolated GIA rates at TG and GPS locations using an available $0.2^{\circ} \times 0.2^{\circ}$ grid of the ICE6G_D model (Peltier et al., 2018, http://www.atmosp. physics.utoronto.ca/ peltier/data.php, last accessed November 18, 2018). Note the GIA predictions are referred to the centre of mass of the solid-Earth (CE) frame, but VLM trends from GPS and ALT-TG are derived with respect to the centre of mass of the entire Earth (CM) system (e.g., Blewitt, 2003). Thus, the GIA discrepancy with respect to our VLM estimates are partly associated with the drift of long-term average CM in the polar direction ( $\mathrm{Wu}$ et al., 2012). 


\section{Methodology}

We further advanced the Kalman-based approach developed by Rezvani et al. (2021) to simultaneously estimate long-term linear and short-term non-linear components of site-specific VLMs as well as geographically correlated altimeter errors through recursive forward and backward solutions. In the forward solution, we used observations up to the computational instant to filter a priori estimates of unknown states and covariances that were inferred from a dynamic model linked to the previous a posteriori estimates. In the backward solution, we smoothed the a posteriori estimates at each instant using all observations available throughout the study span. As the observational series were recorded at different times and locations, computational time steps were adopted from the Jason-series altimeter sampling repeat period ( $\sim 9.9$ days). We considered spatiotemporal correlations between each set of observational series, however correlations between the unknowns were not included. Owing to the close correlations between unknowns across space and time, we approached the ill-posed nature of the problem by estimating our parameters within a refined multi-stage solution as summarized in Figure 1.

\section{Solution 1}

Aim: Derive a priori estimates of linear altimeter systematic error

$>$ Use tight constraints on a priori estimates of linear VLM.

Iterate with updated initial states and uncertainties.

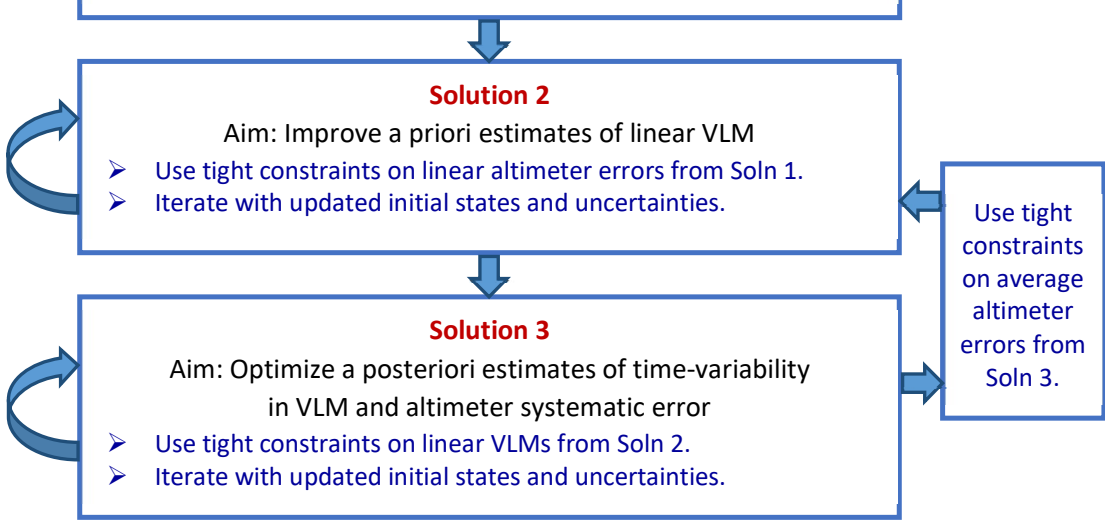

Figure 1. A flow illustration of the multi-stage approach to estimate unknowns within subsequent solutions. The strategy commenced with estimating a priori estimates of linear altimeter systematic error or bias drift from a priori knowledge about linear VLMs at geodetic sites, followed by improving a priori estimates of linear VLM, and concluding with simultaneous estimation of non-linear evolution in altimeter bias drift and VLM estimates in a unified reference frame. 


\subsection{Multiple observational series}

Our input observations included the ALT-TG combinations, ALT differences at tandem/dual crossovers, GPS bedrock heights, and state constraints across the region as described below. We refer to Rezvani et al. (2021) for the underlying observational models.

\subsubsection{ALT minus TG combinations}

We first extracted $1-\mathrm{Hz}$ along-track ASL data from $n_{\mathrm{ALT}}=(1,2, \ldots, l)$ missions at $n_{\mathrm{CP}}=(1,2, \ldots, j)$ ALT CPs, that were then combined with hourly RSL records from $n_{\mathrm{TG}}=(1,2, \ldots, i)$ TGs. We linearly interpolated the RSL in time to the nearest ASL within a distance threshold of $150 \mathrm{~km}$, to construct ALT and TG differences (designated as ATG hereon). We discarded potential outliers as two times the interquartile range (IQR) above or below the medians of ATG residuals (following a mission-specific linear fit). We removed residual annual and semi-annual terms using a harmonic analysis of all ATG observations relating to each CP across the altimeter era, while the mission-specific intercepts were estimated simultaneously. Within our CP selection procedure, we imposed a distance threshold of 20-120 km for the altimeter sampling locations off the coast, and then discarded outlying mission-specific trends that exceeded a threshold of two-times the IQR above or below the median trend from all candidate CPs for any given TG. For later analysis, candidate CPs were flagged as being 'on' or 'off' the continental shelf based on their relative location to the $200 \mathrm{~m}$ depth contour. For computational efficiency, we followed thresholds suggested by Rezvani et al. (2021) to limit our ATG combinations to 8 mission-specific series per TG to select 161, 161, 147, 144, 160, 160, 155, and 154 CPs pertaining to TOPEX-side A/B, Jason-1, Jason-2, Jason-3, ERS-2, Envisat, SARAL, and Sentinel-3A missions, respectively. Note the ATG series of the non-reference missions were incorporated at less frequent sampling periods, e.g., for Envisat, once in every 3rd computational epoch given different repeat orbit periods.

\subsubsection{ALT crossovers}

We constructed measurements of tandem/dual ALT crossover (designated as AXO), through differencing the ASL data of the two overflying missions at the respective CPs. We initially selected the crossover CPs that were located within $500 \mathrm{~km}$ off the coastal gauges. We removed 
outlying observations using two times the IQR above or below the CP-specific, missions-specific medians. For computational cost, we then reduced the numbers of tandem and dual AXO CPs using the same criteria proposed by Rezvani et al. (2021) as well as additional thresholds of RMSE and distance given in Table S2. These limited the number of CPs to 102, 102, 102 and 105 for tandem missions of "TOPEX-side B \& Jason-1", "Jason-1 \& Jason-2", “Jason-2 \& Jason-3” and "ERS-2 \& Envisat", respectively. The number of dual AXO CPs also reduced to 134, 134, 134, 134, 134, 134 and 121 between "ERS-2 \& TOPEX-side A", "ERS-2 \& TOPEX-side B", "ERS-2 \& Jason-1", "Envisat \& Jason-1", “Envisat \& Jason-2", “SARAL \& Jason-2” and "Sentinel-3A \& Jason-3" missions, respectively. We adopted the sampling times of AXO series to the averaged times of ASL measurements from each set of overflying missions. The dual crossover observations were less frequent than the computational intervals of our space-time approach, hence were ingested in a similar way as to the non-reference mission ALT data.

\subsubsection{GPS heights}

With the daily height series from $n_{\mathrm{GPS}}=(1,2, \ldots, k)$ GPS sites, we discarded outliers as three times the IQR above or below the location-specific medians of residuals following a linear fit. We removed instrumental offsets (where they existed) as well as annual and semi-annual signals, that were derived from the trend estimation process within the Hector software version 1.6 (Bos et al., 2013) with a "white plus power-law" noise model (Williams et al., 2004). We then linearly interpolated the resultant GPS series at the measurement times of the nearest ASL series to arrive at third type of input observations. Note the GPS series were effectively decimated (from daily to 2.9-day sampling) to conform with the computational intervals of our space-time approach, that were later used to provide a sanity check on our estimates of VLM trend at GPS locations as compared to those inferred from the Hector analysis using daily sampling.

\subsubsection{State constraints}

We further introduced a suite of pseudo-observation (PSO) constraints to take advantage of preliminary knowledge about the unknowns in different solutions of the multi-stage approach. We defined constraints on linear rates of VLM in the first solution to derive approximate estimates of linear bias drift, that were used in a later solution as time-fixed constraints to examine the a priori velocity field. We constrained the final adjustment to the a posteriori estimates of linear VLM rates 
from the latter solution, to investigate our ability to simultaneously resolve temporal evolution in both bias drift and VLM parameters.

\subsection{Kalman framework}

Our Kalman engine follows the method of Rezvani et al. (2021) except where noted below. The framework has the observation vector $\boldsymbol{z}_{\boldsymbol{q}}=\left[\boldsymbol{z}_{\boldsymbol{q}}^{\mathbf{A T G}} \boldsymbol{z}_{\boldsymbol{q}}^{\text {AXO }} \boldsymbol{z}_{\boldsymbol{q}}^{\text {GPS }} \boldsymbol{z}_{\boldsymbol{q}}^{\text {PSO }}\right]^{T}$ that includes $n_{\mathrm{ATG}}$ ASL minus RSL $\left(\mathbf{z}_{\boldsymbol{q}}^{\mathbf{A T G}}\right)$ series, $n_{\mathrm{AXO}}$ ASL differences at the tandem and dual crossovers $\left(\mathbf{z}_{\boldsymbol{q}}^{\mathrm{AXO}}\right), n_{\mathrm{GPS}}$

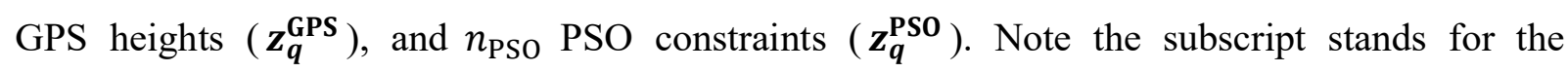
computational timestep $q$ within the Kalman framework, while the superscripts indicate the observation types. We hence allocated $\boldsymbol{x}_{\boldsymbol{q}}=\left[\begin{array}{lllll}\boldsymbol{r}_{\boldsymbol{q}} & \boldsymbol{v}_{\boldsymbol{q}} & \boldsymbol{\delta} \boldsymbol{v}_{\boldsymbol{q}} & \boldsymbol{s}_{\boldsymbol{q}} & \boldsymbol{a}_{\boldsymbol{q}} \\ \boldsymbol{\xi}_{\boldsymbol{q}}\end{array}\right]^{T}$ as the state vector, comprised of (i) "time-fixed" unknowns that include $\boldsymbol{v}_{\boldsymbol{q}}$ for linear VLMs at TG and GPS sites, and $\boldsymbol{s}_{\boldsymbol{q}}$ for across-track ASL slopes; as well as (ii) "time-variable" unknowns that include $\boldsymbol{\delta} \boldsymbol{v}_{\boldsymbol{q}}$ for nonlinear VLMs, and $\xi_{\boldsymbol{q}}$ for time-dependent noise of observations. The intercepts $\boldsymbol{a}_{\boldsymbol{q}}$, defined at initial instants of the observational series, dealt with as either "time-variable" or "time-fixed" parameters, depending on the steps in our multi-stage strategy. The altimeter-specific residual systematic errors or bias drifts $\boldsymbol{r}_{\boldsymbol{q}}$ were primarily assumed to be "time-fixed", however these were treated as "timevariable" quantities in the final optimization of the multi-stage solution approach. In an extension of the method of Rezvani et al. (2021), we differentiated the VLM into linear and non-linear quantities, and further investigated the potential for time-variability in altimeter systematic errors.

We derived filter estimates of the state unknowns $\boldsymbol{x}_{\boldsymbol{q}}$ and covariances $\sum_{\boldsymbol{q}}$ at all computational instants $t_{q}^{\text {Kal }}$ using the forward gains, proceeded with Rauch-Tung-Striebel smoothing to attain the optimum estimates using the backward gains (e.g., Grewal \& Andrews, 2008). We formulated the measurement equation at the timestep $q$ as:

$$
z_{q}=H_{q} x_{q}+e_{q}
$$

with the observational matrix $\boldsymbol{H}_{\boldsymbol{q}}$ constructed on an epoch-by-epoch basis and the Gaussian white noise $\boldsymbol{e}_{\boldsymbol{q}}=\left[\boldsymbol{e}_{\boldsymbol{q}}^{\mathrm{ATG}} \boldsymbol{e}_{\boldsymbol{q}}^{\mathrm{AXO}} \boldsymbol{e}_{\boldsymbol{q}}^{\mathbf{G P S}} \boldsymbol{e}_{\boldsymbol{q}}^{\mathbf{P S O}}\right]^{\boldsymbol{T}}$ accounted for ATG combinations ( $\boldsymbol{e}_{\boldsymbol{q}}^{\mathrm{ATG}}$ ), AXO differences $\left(\boldsymbol{e}_{\boldsymbol{q}}^{\text {AXO }}\right)$, GPS bedrock heights $\left(\boldsymbol{e}_{\boldsymbol{q}}^{\mathbf{G P S}}\right)$ and tight or loose constraints $\left(\boldsymbol{e}_{\boldsymbol{q}}^{\mathbf{P S O}}\right)$, that are each zero-mean and have the variance-covariance (VCV) matrices $\boldsymbol{R}\left(\boldsymbol{e}_{\boldsymbol{q}}^{\mathrm{ATG}}\right), \boldsymbol{R}\left(\boldsymbol{e}_{\boldsymbol{q}}^{\mathrm{AX0}}\right), \boldsymbol{R}\left(\boldsymbol{e}_{\boldsymbol{q}}^{\mathrm{GPS}}\right)$ and $\boldsymbol{R}\left(\boldsymbol{e}_{\boldsymbol{q}}^{\mathbf{P S O}}\right)$, 
respectively. We considered $\boldsymbol{R} \equiv \boldsymbol{R}_{\boldsymbol{q}}$ as the VCV matrix of the measurement noise $\boldsymbol{e}_{\boldsymbol{q}}$, that is notated as:

$$
\operatorname{diag}\left(\boldsymbol{R}_{q}\right)=\left[\boldsymbol{R}\left(\boldsymbol{e}_{q}^{\mathrm{ATG}}\right) \boldsymbol{R}\left(\boldsymbol{e}_{q}^{\mathrm{AXO}}\right) \boldsymbol{R}\left(\boldsymbol{e}_{q}^{\mathrm{GPS}}\right) \boldsymbol{R}\left(\boldsymbol{e}_{q}^{\mathrm{PSO}}\right)\right]^{T}
$$

To form the dynamic model, we linearly linked the state vectors $\boldsymbol{x}_{\boldsymbol{q}}$ and $\boldsymbol{x}_{\boldsymbol{q}-\mathbf{1}}$ at the consecutive instants $t_{q}^{\mathrm{Kal}}$ and $t_{q-1}^{\mathrm{Kal}}$ through:

$$
x_{q}=F x_{q-1}+\varepsilon_{q}
$$

characterized by the diagonal transition matrix $\boldsymbol{F}$, and the Gaussian state process noise $\varepsilon_{q}=\left[\begin{array}{llllll}\varepsilon_{q}^{r} & \varepsilon_{q}^{v} & \varepsilon_{q}^{\delta v} & \varepsilon_{q}^{s} & \varepsilon_{q}^{a} & \varepsilon_{q}^{\xi}\end{array}\right]^{T}$ following the VCV matrix $\boldsymbol{Q} \equiv \boldsymbol{Q}_{\boldsymbol{q}}$, such that:

$$
\operatorname{diag}\left(\boldsymbol{Q}_{q}\right)=\left[\begin{array}{llll}
\boldsymbol{Q}\left(\varepsilon_{q}^{r}\right) \boldsymbol{Q}\left(\varepsilon_{q}^{v}\right) & Q\left(\varepsilon_{q}^{\delta v}\right) \boldsymbol{Q}\left(\varepsilon_{q}^{s}\right) & Q\left(\varepsilon_{q}^{a}\right) & Q\left(\varepsilon_{q}^{\xi}\right)
\end{array}\right]^{T}
$$

Note the superscripts here refer to parameter types for mission-specific bias drifts $\varepsilon_{q}^{r} \sim Q\left(\varepsilon_{q}^{r}\right)$, linear VLMs $\varepsilon_{q}^{v} \sim \boldsymbol{Q}\left(\varepsilon_{q}^{v}\right)$, non-linear VLMs $\varepsilon_{q}^{\delta v} \sim \boldsymbol{Q}\left(\varepsilon_{q}^{\delta v}\right)$, across-track slopes $\varepsilon_{q}^{s} \sim \boldsymbol{Q}\left(\varepsilon_{q}^{s}\right)$, observational intercepts $\varepsilon_{\boldsymbol{q}}^{\boldsymbol{a}} \sim \boldsymbol{Q}\left(\varepsilon_{\boldsymbol{q}}^{\boldsymbol{a}}\right)$ and time-correlated noise $\boldsymbol{\varepsilon}_{\boldsymbol{q}}^{\xi} \sim \boldsymbol{Q}\left(\boldsymbol{\varepsilon}_{\boldsymbol{q}}^{\xi}\right)$.

\subsection{Multi-stage solution approach}

To cope with the high correlation between the weighted average of the VLM across the network and the average bias drifts across the altimeter constellation, we developed the refined multi-stage solution approach as shown in Figure 1 (see Figure S1 for the detailed flow). We first approximated linear estimates of altimeter bias drift, and then improved our GPS-inferred knowledge of linear VLM field across the region. We successively sought to resolve a simultaneous adjustment of nonlinear evolution in both VLM and bias drift unknowns. The linear VLM field at TGs were then updated once the most probable values have been updated from the time-variable bias drifts. The process noise used in our Kalman configuration across the Australian region are provided in the supporting information as referred to below. We return to the challenge of tuning our engine in the discussion. 


\subsubsection{Solution 1}

We commenced our multi-stage approach with "Solution 1" to derive a priori estimates of bias drift per mission using a relatively tight process noise, treating these as linear time-fixed variables. With lack of a priori knowledge, we assumed zero initial-value as the bias drift states for all missions with large uncertainty. We imposed tight constraints on linear VLM trends at GPS sites (from Hector trend analysis) and TG locations (from Kriging of GPS-inferred VLMs within $150 \mathrm{~km}$ of each TG following the procedure by Rezvani et al. (2021), hereon named GPS-Krig), and discarded non-linear VLM contributions. We treated intercepts of ATG and GPS series as time-variable quantities in the presence of possible discrepancy between the a priori and actual VLM trends. We iterated this solution to enhance the convergence and accuracy of the estimates, once the a priori estimates were updated based on the median estimates (for time-fixed variables) and the initial-epoch estimates (for the time-varying quantities). In the iteration, we tuned the process noise of time-variable intercepts in an adaptive fashion following the trends present in the ATG and GPS residuals (see a rule set given in Table S3).

\subsubsection{Solution 2}

We followed with "Solution 2" to improve the a priori estimates of linear VLMs at TG and GPS sites using a relatively loose process noise. We tightly constrained to the resultant median estimates of linear bias drifts from Solution 1, and non-linear VLM contributions were similarly discarded. We fixed the intercepts as time-fixed variables from this solution onward to ensure proper modelling of the temporal evolution in the site-specific VLM parameters. We updated the a priori estimates and repeated this solution to effectively capture long-term localized variability at geodetic sites, once the process noise of time-fixed VLMs were adopted from trends present in the ATG and GPS residuals (see Table S3).

\subsubsection{Solution 3}

In "Solution 3", we sought to explore the potential to simultaneously estimate temporal evolution in both site-specific VLMs and mission-specific bias drifts in a geocentric reference frame, with tight constraints on the median estimates of time-fixed VLM trends at all geodetic sites generated from Solution 2. As our linear VLM field has been updated in the former solution, we once again estimated bias drifts. We used relatively loose process noise, zero initial-value, and large uncertainties for the unknown states of bias drifts and non-linear VLMs. This solution was 
iterated once the process noise of non-linear VLM was tuned to suitably separate the short-term localized variability at geodetic sites from evolving bias drifts (Table S3).

The datum continuity of TG records is fundamentally required to provide reliable estimates of VLM and ASL trends (e.g., Wöppelmann et al., 2008). We detected outlying ATG observations contaminated with substantial RSL datum issues using the same approach proposed by Rezvani et al. (2021), which were excluded from the final solution (see Figure S4). We subsequently ran Solution 2 once more to update the VLM trends at TGs, relative to the weighted averages of evolving bias drift as the most probable estimates from Solution 3.

To define appropriate process noise for the time-variable evolution in VLM and bias drift in the iteration of Solution 3, we first tuned the filter based on trends in the height residuals to derive non-linear VLM at GPS sites (Table S3), such that our continent-wide (coastal) stacked time series closely follows an external stack from raw GPS heights that were detrended outside of our engine (Figure 4). We then selected a relatively loose process noise for bias drift parameters to appropriately capture any temporal evolution over the altimeter era, driven by either non-linearity in mission-specific errors or common-mode variability of TG VLMs, and short-term components of VLMs were constrained to be zero at all geodetic sites (see Figures S17 and S18 in the case of multi-mission and reference-mission solutions, respectively). We finally proceeded to tune the filter to yield comparable variability in non-linear VLM at TGs, such that the dispersion of the averaged stacked non-linear VLM from TGs closely matches that from our non-linear VLMs at near coastal GPS sites (Figure 4). We assumed GPS heights were indicative of surface loading deformation, hence we expected comparable variability at TG sites. We later return to discuss advantages and potential limitations of this scheme in extracting site-specific loading displacements in the discussion.

Note that a priori values of the across-track ASL slopes were derived from the DTU15 mean sea surface model, and these parameters were then tightly constrained to the medians of a posteriori estimates from the former solutions. We modelled the correlated noise with zero initial value and large uncertainty, and the respective process noise was adopted from power spectrum analysis (Section 3.4.1). The AXO intercepts, determined from the medians of the observational series, were similarly introduced as tight constraints to assist in retrieving linear altimeter errors in 
conjunction with the ATG observations in all solutions, except for the last optimization where these were updated given non-linear estimates of bias drift.

\subsection{Preliminary steps}

We undertook preliminary analyses to configure the noise content and covariances of observations within our Kalman framework. We considered some practical schemes to cope with implementation issues as well.

\subsubsection{Spectral noise analysis}

We used Lomb-Scargle power spectra of observational residuals (after removal of linear fit, Figure S2) to determine the noise content at measuring locations in terms of time-dependent and time-independent errors (e.g., Buttkus, 2000). We approximated time-correlated noise of observations to the first-order autoregressive (AR1) sequence and defined the "white plus AR1" stochastic model for the ATG, dual AXO and GPS, but a "white-only" model for the tandem AXO series. We assumed the residual oceanography between the TG and CP measurement locations as a stationary process over the satellite era, and the time-correlated noise were thus estimated by stacking ATG combinations from all missions overflying a specific CP.

Within this process, we specified variances of time-independent noise populated in the diagonal terms of the ATG $\boldsymbol{R}\left(\boldsymbol{e}^{\mathrm{ATG}}\right) \equiv \boldsymbol{R}\left(\boldsymbol{e}_{\boldsymbol{q}}^{\mathrm{ATG}}\right)$, AXO $\boldsymbol{R}\left(\boldsymbol{e}^{\mathrm{AXO}}\right) \equiv \boldsymbol{R}\left(\boldsymbol{e}_{\boldsymbol{q}}^{\mathrm{AX0}}\right)$ and GPS $\boldsymbol{R}\left(\boldsymbol{e}^{\mathrm{GPS}}\right) \equiv \boldsymbol{R}\left(\boldsymbol{e}_{\boldsymbol{q}}^{\mathrm{GPS}}\right)$ covariance matrices. We further determined magnitudes of time-dependent noise (populated in the diagonal elements of the $\boldsymbol{Q}\left(\boldsymbol{\varepsilon}^{\xi}\right) \equiv \boldsymbol{Q}\left(\boldsymbol{\varepsilon}_{\boldsymbol{q}}^{\xi}\right)$ VCV matrix), in conjunction with the AR1 correlation coefficients (populated in the relevant elements of the diagonal $\boldsymbol{F}$ transition matrix). The remaining elements in the transition matrix defined the temporal evolution of parameters through randomwalk processes as described in Figure S1.

\subsubsection{Semi-variogram analysis}

We used semi-variogram analysis to determine the similarity between adjacent samples in the random fields varying across time and space (e.g., Montero et al., 2015). We first computed empirical semi-variances of observational residuals of ASL, RSL and GPS height that were measured at paired locations. We subsequently extracted the spatiotemporal covariances from 
Gaussian negative-definite semi-variogram models fitted to the resultant semi-variances at temporal slices up to 10 days (Figure S3). We finally computed the covariances within ATG and AXO noise by propagating random errors. We populated these in the off-diagonal elements of the $\mathrm{ATG} \boldsymbol{R}\left(\boldsymbol{e}^{\mathrm{ATG}}\right) \equiv \boldsymbol{R}\left(\boldsymbol{e}_{\boldsymbol{q}}^{\mathrm{ATG}}\right), \mathrm{AXO} \boldsymbol{R}\left(\boldsymbol{e}^{\mathrm{AXO}}\right) \equiv \boldsymbol{R}\left(\boldsymbol{e}_{\boldsymbol{q}}^{\mathrm{AXO}}\right)$, and GPS $\boldsymbol{R}\left(\boldsymbol{e}^{\mathrm{GPS}}\right) \equiv \boldsymbol{R}\left(\boldsymbol{e}_{\boldsymbol{q}}^{\mathrm{GPS}}\right)$ covariance matrices up to the length-scales of 750, 750 and $350 \mathrm{~km}$, respectively.

\subsubsection{Practical schemes}

As we adopted the time base defining each computational step of the Kalman solutions to the 7.9-day sampling of the Jason-series missions, we could incorporate observations from ALT, TG and GPS that were recorded at different times and locations. We updated the predicted and filtered estimates of the unknown states and covariances if the associated observations were included in the individual timesteps, otherwise the former estimates of the unknowns and uncertainties were carried forward. We used singular value decomposition to invert the large-dimension covariance matrices in the underlying solutions across the Australian study region, with the matrix sizes of $\sim 4000 \times 4000$ and $\sim 7000 \times 7000$ for the filtering and smoothing gains, respectively. In terms of processing time, our computations (based on 1560 ATG, and 1336 AXO, and 210 GPS observations) were processed on two nodes each with 28 available CPUs (with clock speed well under contemporary Intel Xeon frequencies) and 128 GB of memory, requiring 7.48 (7.16), 8.88 (6.87) and 14.04 (13.56) hours for Solutions 1, 2 and 3 (and the respective iterations in brackets), respectively.

\section{Results}

We first applied the developed methodology to infer long-term VLM estimates at geodetic sites around the Australian study region. We made a comparison between VLM trends from our multimission solution with estimates inferred from spatially interpolated GPS (GPS-Krig) and predicted GIA alternatives. We subsequently investigated the feasibility of observing short-term variability in both VLMs and bias drifts, in the same framework. We explored the spatial coherence of ASL rates at TGs computed using our VLM trends (with and without applying the bias drift corrections), in contrast to those derived using the GPS-Krig and GIA alternatives. We assessed the (expected) agreement between the ASL rates at the TGs and ALT CPs around the region. We then undertook 
a suite of sensitivity tests to understand the dependence of our estimates on a priori configurations and assumptions, assess the improvements of the multi-mission over the reference-mission-only implementation and investigate the sensitivity of results to CPs located off the continental shelf. We finally evaluated the performance of the Kalman solution through probing a posteriori estimates of unknowns and residuals.

\subsection{Linear VLM}

Figure 2a shows the magnitudes and spatial distribution of our VLM trends at TGs and nearby GPS sites, estimated using multi-mission datasets from Solution 2 of the multi-stage approach (Figure 1). As an initial inspection, we found a good agreement between our linear VLM estimates and Hector-derived trends at GPS sites, with a weighted mean difference of $+0.03 \mathrm{~mm} / \mathrm{yr}$ and WRMSE of $0.12 \mathrm{~mm} / \mathrm{yr}$. This comparison identified no statistically significant difference, confirming the basic operation of the filter. Conversely, we noticed $22 \%$ of TGs (flagged by green labels in Figures 2 and 3) with significant differences at the 1-sigma confidence level between our VLM trends and spatially interpolated GPS velocities (with a weighted mean difference of +0.06 $\mathrm{mm} / \mathrm{yr}$ and WRMSE of $0.73 \mathrm{~mm} / \mathrm{yr}$ ). The scatter of differences at TGs was reduced from 0.74 to $0.58 \mathrm{~mm} / \mathrm{yr}$ when these 5 very localized anomalies were excluded.

Figure $2 \mathrm{~b}$ shows a map of the differences between our VLM trends and the benchmark estimates (i.e., GPS-Krig at TG, and Hector at GPS, locations). These VLM differences are shown in Figure 3 , as a function of latitude across the region. From these figures, the VLM rate discrepancies between GPS and TG sites are almost all within $\pm 1.5 \mathrm{~mm} / \mathrm{yr}$. We repeated the same comparison between our VLM estimates and the ICE6G_D predictions (see Figures S5 and S6). The weighted mean differences of our solution minus the GIA predictions were -0.42 and $-0.40 \mathrm{~mm} / \mathrm{yr}$ at TG and GPS sites, with the respective WRMSE of 0.93 and $1.04 \mathrm{~mm} / \mathrm{yr}$. Tables S4 and S5 list a priori and posteriori estimates of VLM at TG and GPS sites, respectively.

We compared the estimates of VLM uncertainty from our solution, scaled by the a posteriori variance factor, with those from GPS-Krig and Hector approaches (Figure S7). The average uncertainties from our approach at GPS and TG sites were 0.65 and $0.71 \mathrm{~mm} / \mathrm{yr}$, respectively, 
comparable with 0.83 and $0.87 \mathrm{~mm} / \mathrm{yr}$ inferred from Hector-derived (at GPS) and GPS-Krig (at TG) uncertainties, respectively.

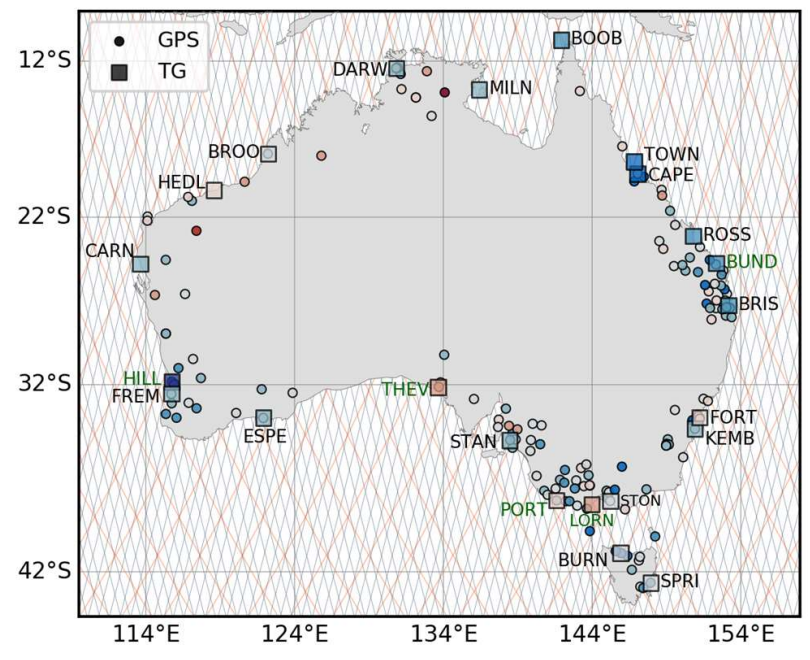

(a) Our VLM estimate

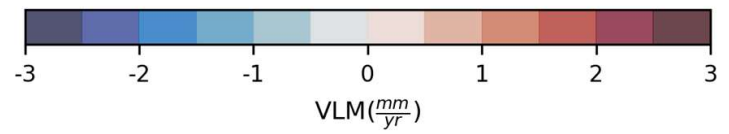

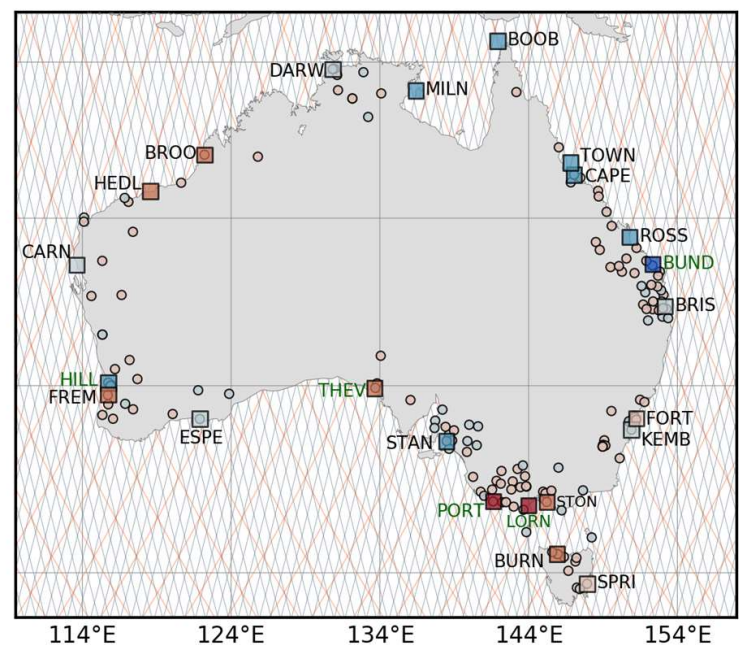

(b) VLM difference

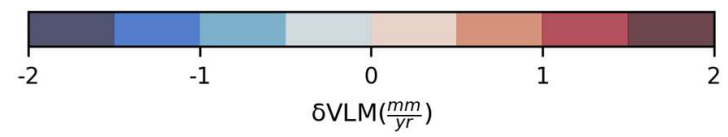

Figure 2. Map of (a) our estimates of linear vertical land motion (VLM) using multi-mission datasets, and (b) differences of our approach minus Global Positioning System (GPS)-Krig and Hector alternatives at tide gauge (TG, squares) and GPS sites (circles), respectively. TGs with significant differences at 1-sigma are annotated in green. For clarity, TG latitudes at the TOWN and FREM locations are shifted by +0.75 and -0.45 degrees, respectively. The ground tracks of Jason-series and Envisat-series altimeters are shown in orange and cyan, respectively.

The GPS-inferred VLM field showed that the Australian plate is largely subsiding with weighted mean rates of $-0.10,-0.38,-0.95$ and $-0.62 \mathrm{~mm} / \mathrm{yr}$ in the NW, NE, SW and SE regions, respectively, in general agreement with previous GPS estimates of subsidence (Hammond et al., 2021; Riddell et al., 2020). The estimates at TGs revealed localized VLM trends around the continent that are not completely consistent with the GPS-Krig interpolations. For example, we found that owing to the groundwater extraction in the Perth basin the current subsidence at the FREM TG (VLM rate of $-0.96 \pm 0.53 \mathrm{~mm} / \mathrm{yr}$ ) is noticeably closer to zero than the PERT GPS ($3.02 \pm 0.48 \mathrm{~mm} / \mathrm{yr}$, with a record span of $25 \mathrm{yrs}$, and $\sim 31 \mathrm{~km}$ away), the HIL1 GPS $(-2.17 \pm 0.55$ 
$\mathrm{mm} / \mathrm{yr}$, a record span of $22 \mathrm{yrs}$, and spaced by $\sim 25 \mathrm{~km})$, and the HILL gauge $(-2.38 \pm 0.52 \mathrm{~mm} / \mathrm{yr}$, $\sim 25 \mathrm{~km}$ away), which is broadly consistent with the findings of Featherstone et al. (2015). Our results indicated that VLM at the FORT TG $(+0.04 \pm 0.77 \mathrm{~mm} / \mathrm{yr})$ in Sydney Harbour is marginally yet insignificantly different from that at the KEMB TG $(-0.64 \pm 0.67 \mathrm{~mm} / \mathrm{yr}$ with a $\sim 75 \mathrm{~km}$ separation), and the FTDN GPS ( $-0.61 \pm 0.59 \mathrm{~mm} / \mathrm{yr}$, with shorter timespan of $\sim 7 \mathrm{yrs}, \sim 1 \mathrm{~km}$ away). These sites are however potentially limited by the narrow shelf width in this region and the proximity to the influence of the intensifying East Australian Current (we return to this issue later in detail).

We observed that the TOWN TG (VLM rate of $-1.75 \pm 0.88 \mathrm{~mm} / \mathrm{yr}$ ) is subsiding slightly faster (insignificant) than the CAPE TG $(-1.54 \pm 0.73 \mathrm{~mm} / \mathrm{yr}$, separated by $\sim 24 \mathrm{~km})$, both faster than the TOW2 GPS ( $-0.85 \pm 0.50 \mathrm{~mm} / \mathrm{yr}, \sim 23 \mathrm{~km}$ away, and spanning $\sim 25 \mathrm{yrs})$. An anomalous uplift of $+0.95 \pm 0.71 \mathrm{~mm} / \mathrm{yr}$ was also found at the LORN TG, compared with a subsidence of $-0.15 \pm 0.70$ $\mathrm{mm} / \mathrm{yr}$ from the nearest TG at the STON location ( $\sim 10 \mathrm{~km}$ away), and $-0.67 \pm 0.59 \mathrm{~mm} / \mathrm{yr}$ at the nearest GPS at the MNGO location ( $\sim 39 \mathrm{~km}$ away, and spanning $\sim 8 \mathrm{yrs})$. Interestingly, LORN (one of the ABSLMP supplementary stations) shows the least rate of RSL rise (BOM monthly report, http://www.bom.gov.au/ntc/IDO60201/IDO60201.202108.pdf), supporting its potential localised uplift (or highly atypical localised oceanographic setting).

The extent to which these VLM rates are statistically significant and reliable is an important question. Some of the differences may be caused by either undetected datum issues smaller than our detection resolution ( $\sim 15 \mathrm{~mm}$ depending on temporal location in the record) or affected by residual oceanographic signals between the TG and ALT (CP) sampling locations. We return to this point later by evaluating the spatial coherence in the resultant ASL estimates (Section 4.4), as well as the spatial variability of the ATG noise pertaining to each gauge (Section 4.6) and impact of ALT sampling (Sections 4.5 and 4.6). 


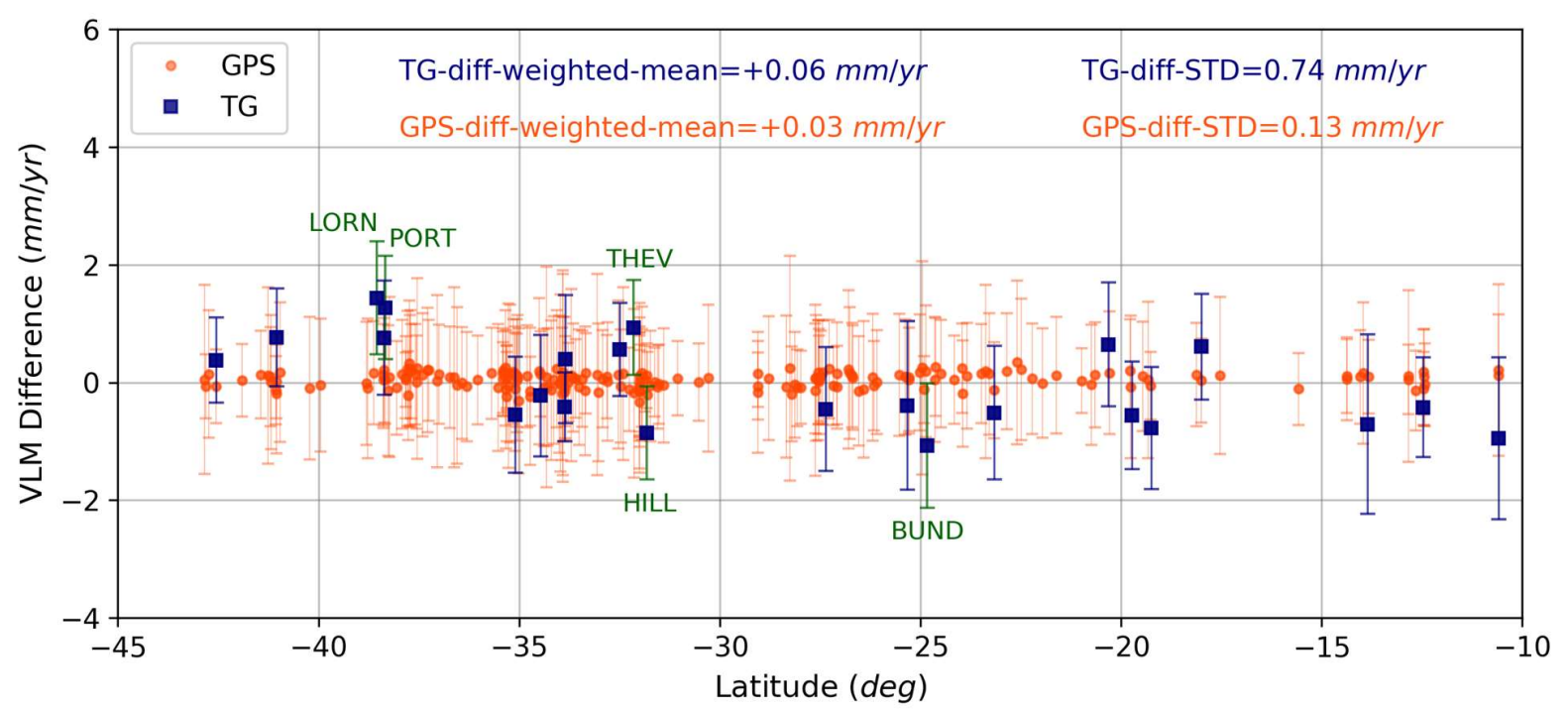

Figure 3. Profile of vertical land motion (VLM) differences plotted against latitude, our estimates minus Global Positioning System (GPS)-Krig at tide gauges (TGs, blue squares) and our estimates minus Hector at GPS sites (orange circles). Sites with significant differences at 1-sigma are labelled in green. Error bars are \pm 1 -sigma and scaled by the a posteriori variance factor. For clarity, TG latitudes at the CAPE, CARN and FREM locations are shifted by -0.45 degrees.

\subsection{Temporal variability in VLM}

We next sought to determine whether our approach has the fidelity to simultaneously resolve potential non-linearity in crustal motion or bias drift. To tune our filter, we derived the average stack of our multi-mission estimates of time-variable TG VLMs and compared it to that from coastal GPS sites as our benchmark (Figure 4). Approximately $75 \%$ of our selected GPS are within $60 \mathrm{~km}$ of the coast - we make the broad assumption that these sites would be subject to comparable low-frequency time-variable mass loading conditions as the TGs. As an external control, we also checked our stacked time series with the stack from the original raw GPS height series that were detrended outside of our engine (Figure 4). We obtained all stacked series from Huber Robust estimation using iteratively reweighted least squares (IRWLS, e.g., Maronna et al., 2006; Rezvani et al., 2015). 


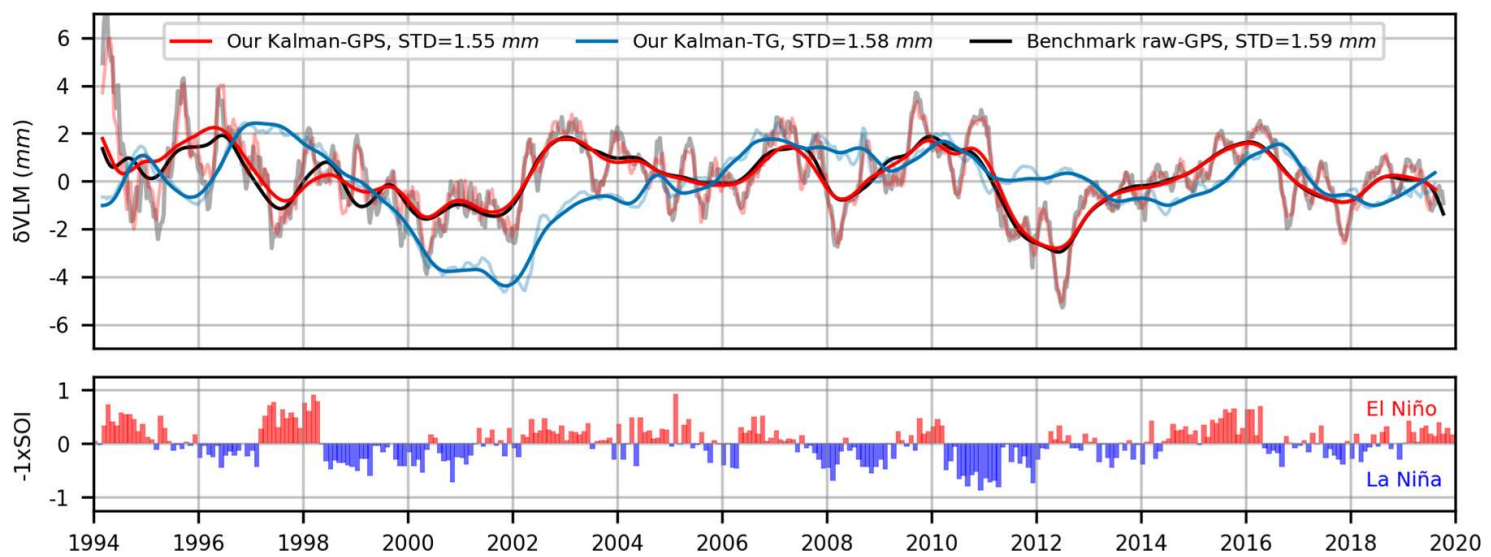

Figure 4. Weighted average stack of our estimates of non-linear VLMs at tide gauges (TGs, blue line) and coastal Global Positioning System (GPS) sites (red line), with respect to the control stack derived from detrended raw height series (black line), around the Australian continent. The smoothed lines show the lowpassed results after applying a Butterworth filter to the stacked series. For comparison, Southern Oscillation Index (SOI) with the sign reversed is shown in the lower panel as the climatic descriptor in the region. The annotated standard deviations (STDs) indicate the comparable variability between these stacks.

Our GPS-stacked time series closely followed the external raw stack (correlation coefficient of +0.96 ). The time-variability in the stacked GPS series at the few-mm level is likely to be mainly driven by changes in Terrestrial Water Storage (TWS) around the continent, which is in turn modulated by the major climate modes of the El Niño-Southern Oscillation (ENSO) and the Indian Ocean Dipole (IOD) anomaly (e.g., Fasullo et al., 2013). We observed some correlation between the GPS-stacked time series and the Southern Oscillation Index (SOI) used as a proxy for ENSO (Figure 4). We note this correlation $(-0.22)$ is weak, yet it is broadly consistent with our expectation that the hydrological loadings are the dominant driver of non-linear VLM in GPS around Australia (e.g., Han, 2017; McGrath et al., 2012; Tregoning et al., 2009). The broader climatic drivers of this signal are complex and their interaction likely to be non-linear (e.g., Fasullo et al., 2013)

The multi-mission TG-stacked series showed some correlation with our independent GPS stack (correlation coefficient of +0.34 ). This suggests that the technique had some skill in the determination of non-linear TG VLMs driven by the continent-wide surface loading deformation. This further provided the opportunity to investigate the evolution of regional altimeter systematic errors in the simultaneous solution. Site-by-site comparisons of non-linear VLMs, however, 
revealed high variability between some TGs and their nearby GPS. On closer inspection, we found close correlation between the variability of the estimated time-variable VLM at TGs and the ATG noise magnitude which is likely dominated by residual oceanographic signals between TG and ALT (CP) sample locations (Figure 10). This remains a key limitation in fully resolving any subtle non-linear signals in VLM, including subtle post-seismic relaxation (Riddell et al., 2021), at a specific TG using this approach. We further return to this point to examine the spatial variability and potential relationship of these with distances between CPs and TGs, as well as proximity/geometry to the coast and the level to which CPs are located on the continental shelf in Section 4.6.

\subsection{Temporal variability in altimeter systematic errors}

As a further goal of this study, we investigated potential non-linearity in regionally-coherent altimeter systematic errors in a simultaneous solution while non-linear TG VLMs were estimated. Figure 5 shows the resultant estimates of systematic errors in the multi-mission altimetry products around the Australian coast. The average values of these estimates, ranging from $-1.09 \pm 0.14$ to $+4.80 \pm 0.26 \mathrm{~mm} / \mathrm{yr}$, suggest that altimeter-specific bias drifts are significant in a regional context, and remain within mission specifications.

The investigation into time variability of altimeter-specific errors offered interesting insight, particularly for the Jason-2 mission. Figure 5 shows a significant change in behaviour in the first $\sim 3.5$ years of the Jason-2 performance which was further supported by analysis of the absolute bias series from the Bass Strait altimeter validation facility (e.g., Watson et al., 2020). On first inspection, this anomaly was suspected to have arisen in our engine due partly to imperfect crosscalibration of Envisat and Jason-2 up until 2010.8 when Envisat ceased. A solution with the reference-mission-only data, however, yielded a similar pattern for Jason-2 drift (Figure S15), confirming it was not the partial overlap with Envisat causing the perturbation. 

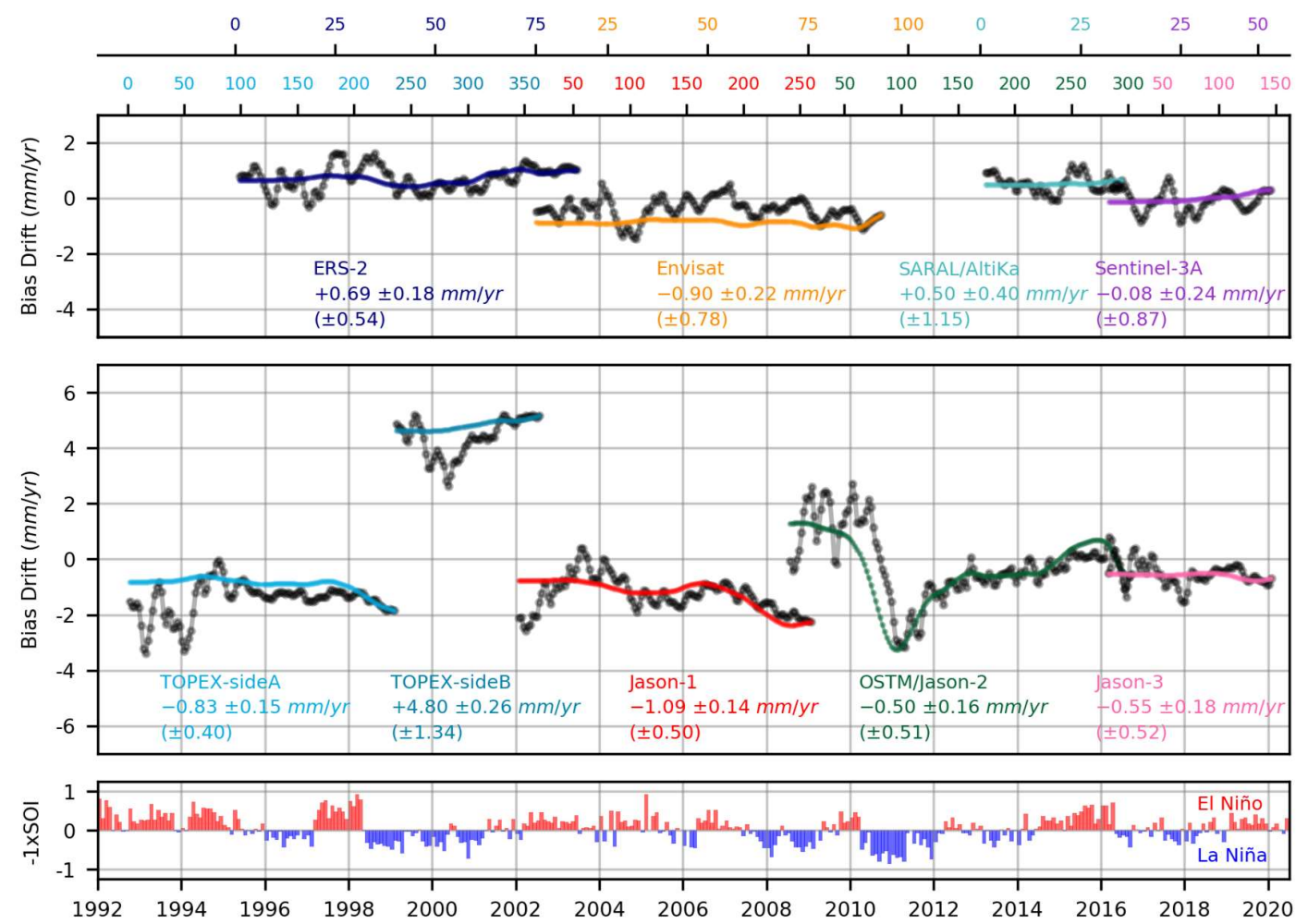

Figure 5. Time-variable systematic errors of (top) non-reference and (middle) reference altimeters over the study region, estimated simultaneously from Solution 3. Note the convergence between the estimates from filtered (black lines) and smoothed (coloured lines) solutions. The mission-specific averages of smoothed bias drifts are annotated with the 1-sigma uncertainties that have been scaled by the a posteriori variance factor. The filter-based rate uncertainties are given in brackets. The sign-inversed Southern Oscillation Index (SOI) is shown in the lower panel. The non-reference and reference cycles are annotated on the top axes with the same colours.

Weak correlation with the dominant climate mode potentially suggests that climate drivers are plausible. It is notable that the 2010-12 La Niña period was remarkable over the Australian continent, with the large amount of water mass on the continent clearly affecting global mean sealevel (e.g., Boening et al., 2012). The signal we see in Jason-2 could potentially arise due to a common differential steric effect between the TG and CPs (associated with enhanced atypical continental runoff/discharge for example). Alternatively, this artefact may be associated with an inadequate resolution used in the time-variable gravity field used for the Jason-2 orbit determination (e.g., Beckley et al., 2012). In both cases, the lack of comparable La Niña events and the finish of the Envisat mission prior to the major signal in Jason-2 makes this difficult to 
resolve. Other drivers also cannot be excluded (Belli et al., 2021; Couhert et al., 2018). We further return to this effect in the discussion.

\subsection{Implications for coastal and offshore ASL}

We applied multi-mission estimates of linear VLM to RSL rates determined from the TG data resampled from the non-tidal residuals every $\sim 9.9$ days, to derive ASL trends. These VLMs were derived from Solution 2 as the non-linear VLMs were discarded, and tightly constrained to the weighted averages of linear bias drifts from Solution 1 (see Figure 1). These averaged systematic errors are typically small but significant (ranging from $-1.95 \mathrm{~mm} / \mathrm{yr}$ for Jason- 1 to $+3.88 \mathrm{~mm} / \mathrm{yr}$ for TOPEX-side B, with a discrepancy of $\pm 0.04-0.92 \mathrm{~mm} / \mathrm{yr}$ compared with the averaged estimates from final solution shown in Figure 5). We ran an appropriate Kalman framework with a "white plus AR1" stochastic model to estimate the RSL trends over the same timeframe of the altimetry records. We used spectral analysis to derive tuning parameters including the measurement noise, as well as the process noise and transition coefficients of the time-correlated errors. We selected tight process noise of $10^{-3} / \sqrt{9.9} \mathrm{~mm} / \mathrm{yr} \sqrt{\mathrm{s}}$ and $10^{-6} / \sqrt{9.9} \mathrm{~mm} / \sqrt{\mathrm{s}}$ (where $\mathrm{s}$ is the 9.9-day Kalman timestep) to tune the estimates of linear trends and intercepts, respectively. We considered spatiotemporal covariances within the RSL noise from semi-variogram analysis, up to a length-scale of $750 \mathrm{~km}$ (Figure S3).

We observed spatial inconsistency in the RSL trends at adjacent TGs (Figure 6a), which we speculated has a dominant contribution from localized VLM processes and not localised ocean processes given the spatial scale involved and general connectivity of the gauge locations to the open ocean. Conversely, inspection of the computed ASL trends showed the expected improved coherence at nearby TGs around the coast (Figure $6 \mathrm{~b}$ ). Over a data duration of $\sim 27 \mathrm{yrs}$, we expected relatively strong regional correlation in the ASL trends, noting the potential for increased sea-level rise in the North and North-West regions due to mostly ENSO-related influences (White et al., 2014). Our approach yielded more spatially coherent ASL estimates than those computed using GPS-Krig and GIA VLM (compare Figures $6 \mathrm{~b}$ and S23). Based on our assumption, this suggests that localized VLM trends at the TGs cannot be reliably inferred from either spatially interpolated 
GPS or GIA outputs - each of which would result in an inadequate representation of likely ASL variability in the region. Table S4 lists the RSL and ASL estimates at the TG locations.

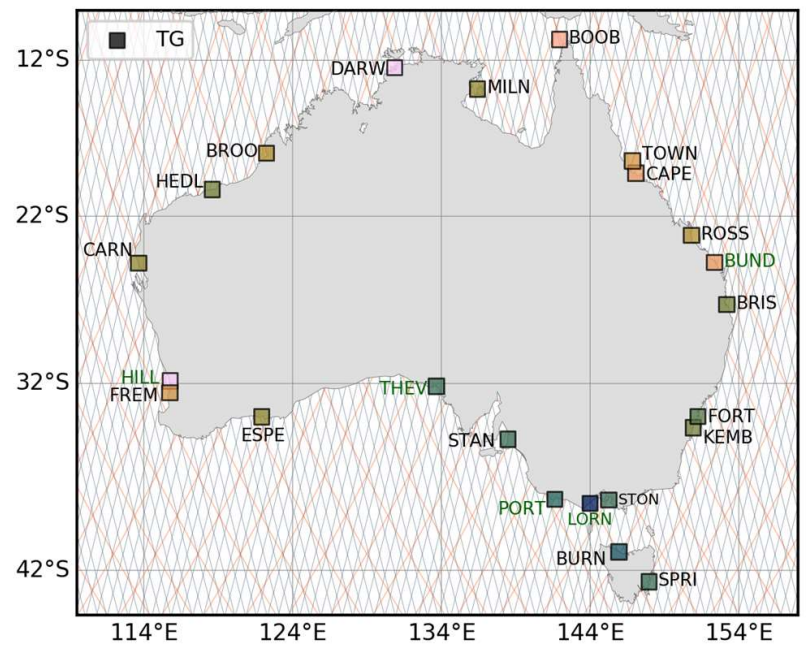

(a) RSL estimate

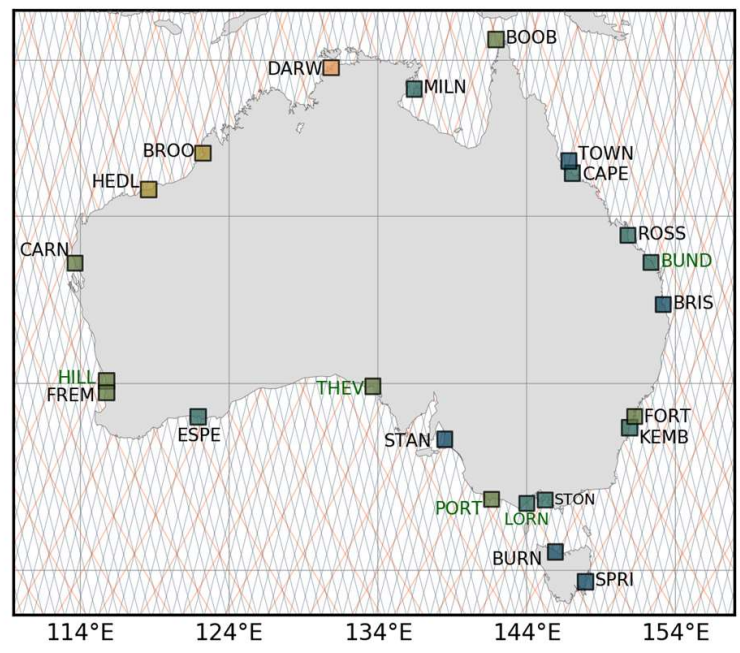

(b) Our ASL estimate

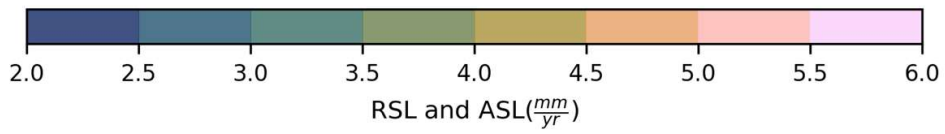

Figure 6. Map of (a) relative sea-level (RSL) trends, and (b) resultant absolute sea-level (ASL) rates at tide gauge (TG) locations after applying our multi-mission estimates of linear vertical land motion (VLM), over the same timeframe (1992.7 to 2020.1). TGs with 1-sigma significant VLM differences from GPS-Krig interpolations are annotated in green. For clarity, TG latitudes at the TOWN and FREM locations are shifted by small amounts. The ground tracks of Jason-series and Envisat-series altimeters are shown in orange and cyan, respectively.

We also revisited the resultant ASL trends at TGs after the time-variable altimeter errors were estimated from Solution 3, resolving both linear and non-linear parts of VLM over the whole satellite era (see Figure 1). We once again ran Solution 2 to update the multi-mission TG VLM trends, with tight constraints on the weighted averages of the evolving bias drifts annotated in Figure 5. We found a negligible discrepancy between our revisited and former estimates of TG VLM, with a weighted average difference of $+0.01 \mathrm{~mm} / \mathrm{yr}$ and the WRMSE of $0.04 \mathrm{~mm} / \mathrm{yr}$ (see Figure S8), which led to an insignificant improvement of the coherence in ASL changes around the region. This indicates that our a priori VLM trends were sufficiently reliable to provide a reasonably stable datum for the estimation of the time-fixed bias drifts. This further suggests that 
considering non-linear VLM has a relatively negligible role in estimating the average bias drift over any one mission in this region.

To examine the spatial variability in the sea-level rise, we fitted a quadratic polynomial to the underlying sets of ASL estimates as a function of latitudes in the SE-NW direction (hereon referred to as the reduced latitudes, Figure 7). The RMSE of our ASL trends about the fitted model is 0.39 $\mathrm{mm} / \mathrm{yr}(0.37 \mathrm{~mm} / \mathrm{yr}$ using the revisited VLMs), compared with 0.67 and $0.75 \mathrm{~mm} / \mathrm{yr}$ from GPSKrig and GIA estimates. This implies a $\sim 42 \%$ and $\sim 48 \%$ reduction in variability of the geographical ASL trends (referred to the SE-NW direction), respectively. We found departures of our ASL estimates at TGs from the fitted quadratic polynomial up to a maximum of $\sim \pm 0.8 \mathrm{~mm} / \mathrm{yr}$. Our estimates suggest a weighted average ASL rate of $+3.40 \pm 0.34 \mathrm{~mm} / \mathrm{yr}$ (slightly higher than $+3.31 \pm 0.31 \mathrm{~mm} / \mathrm{yr}$ from the revisited VLMs) around the Australian coast with the highest values at individual TGs in the NW (around $\sim 5.0 \mathrm{~mm} / \mathrm{yr}$ ) and the lowest in the SE (around $\sim 2.5 \mathrm{~mm} / \mathrm{yr}$ ).

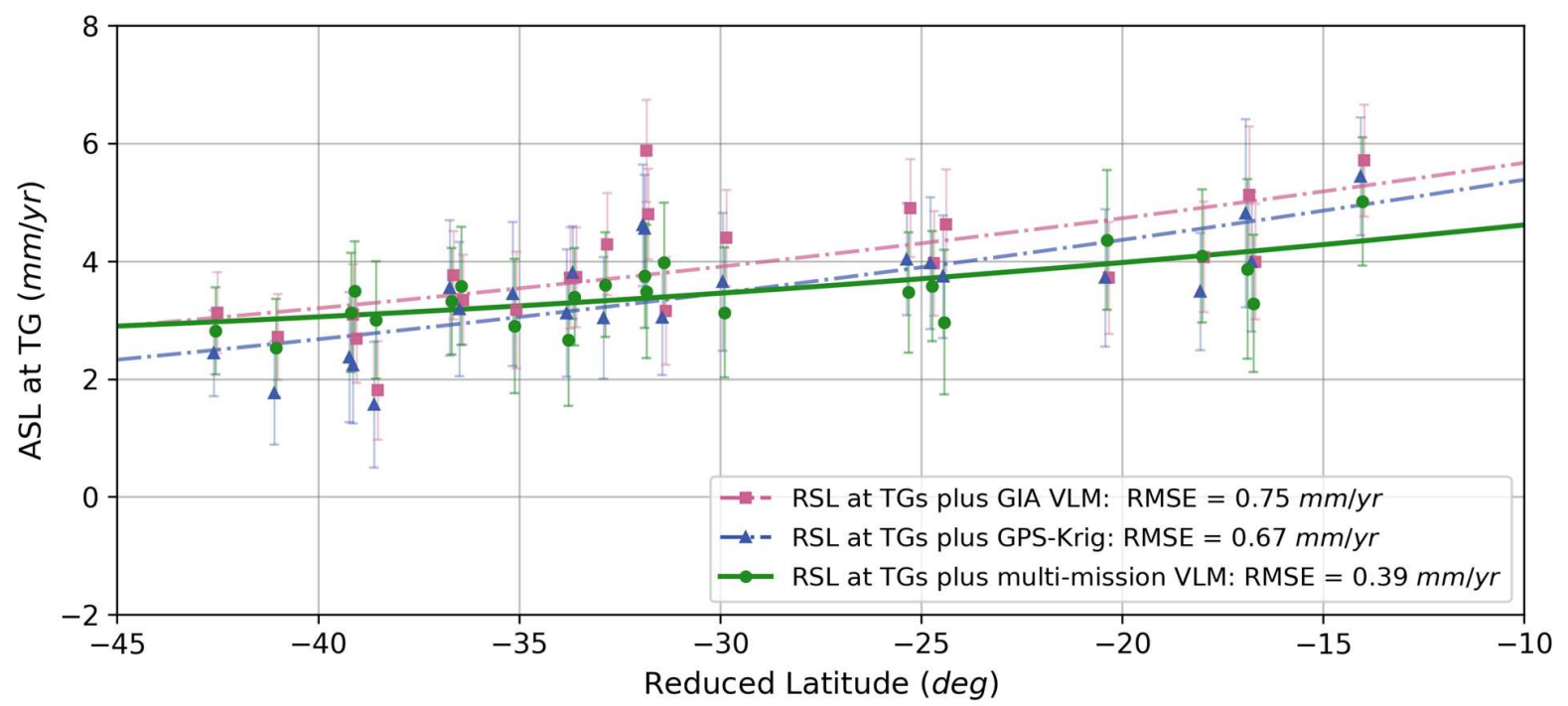

Figure 7. Profile of absolute sea-level (ASL) trends at tide gauges (TGs), comparing estimates derived using relative sea-level (RSL) plus our multi-mission VLMs (green circles) with those from Global Positioning System (GPS)-Krig (blue triangles) and ICE6G_D Glacial Isostatic Adjustment (GIA, pink squares) values. Solid and dashed lines show a quadratic polynomial fitted to each set of ASL estimates per latitude reduced to the SE-NW direction with root mean squared error (RMSE) about this fit annotated in the legend. Error bars are \pm 1 -sigma scaled by the a posteriori variance factor. Note some TG locations have been shifted horizontally by small amounts for clarity. 
For further investigation, we evaluated the consistency of our TG ASLs with ASLs at CPs computed from altimetry data alone. To undertake this assessment, we compared our TG ASL with ASL derived from Jason- and Envisat-series data at each CP used. We computed the altimetry ASL outside of our engine yet applied our corrections for time-varying mission-specific bias drifts (Figure 5) and relative intra- and inter-mission biases (Figures S21 and S22). We ran a suitable Kalman platform to derive the ASL estimates at the altimetry CPs in the satellite era. We undertook spectral analysis to derive the measurement noise, the process noise of time-correlated errors, and the AR1 transition coefficients. We assumed the same process noise used from RSL trend analysis to estimate the linear trends and CP-specific intercepts. Spatiotemporal covariances within the ASL noise were also applied up to a length-scale of $750 \mathrm{~km}$, from the semi-variogram analysis (Figure S3).

As expected, we found a good agreement between our ASL estimates at TG and CP locations, confirming the geographical dependence in ASL rise, relative to the SE-NW direction in the region (Figure 8). The inferred ASL estimates at altimetry CPs show RMSE of $0.46 \mathrm{~mm} / \mathrm{yr}$ once fitted with a polynomial reduced to the SE-NW direction, and suggested the weighted average ASL rate of $+3.51 \pm 0.26 \mathrm{~mm} / \mathrm{yr}$. Comparing our individual estimates of coastal ASL at TGs and offshore ASL at the nearest CPs reveals differences of $\sim \pm 1.1 \mathrm{~mm} / \mathrm{yr}$. This range reached up to $\sim \pm 1.8 \mathrm{~mm} / \mathrm{yr}$ and $\sim \pm 2.3 \mathrm{~mm} / \mathrm{yr}$ if the GPS-Krig and GIA estimates of VLM were substituted to derive ASL trends at TGs, respectively. This comparison supports the use of our VLMs for sea-level studies, under the assumption of zero trend in the ATG differences (of oceanographic origin) over the duration of the data. 


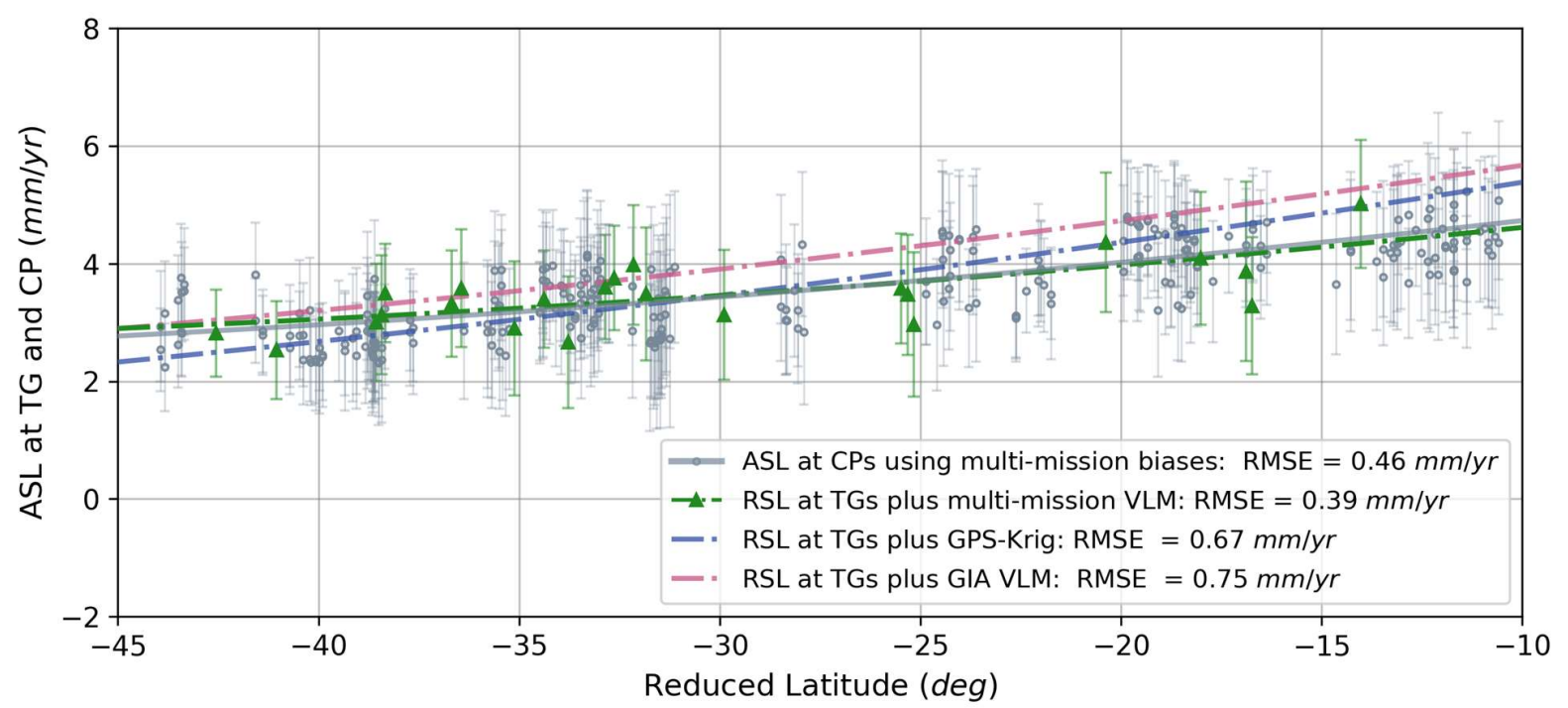

662

Figure 8. Profile of absolute sea-level (ASL) trends at tide gauges (TGs) using relative sea-level (RSL) plus our multi-mission vertical land motions (VLMs), Global Positioning System (GPS)-Krig trends and Glacial Isostatic Adjustment (GIA) predictions, compared with those derived at comparison points (CPs) using altimetry alone after applying estimated bias drifts and relative biases. Solid and dashed lines show a quadratic polynomial fitted to each set of ASL estimates per latitude reduced to the SE-NW direction with root mean squared error (RMSE) annotated. Error bars are \pm 1 -sigma scaled by the a posteriori variance factor.

\subsection{Sensitivity assessment}

We undertook a suite of experiments to assess the sensitivity of the method to the assumptions and configurations considered. First, we compared the performance of the multi-mission solution to the case when reference-mission-only data was used, providing insight into the advantage of the expanded dataset. As mentioned in Section 4.3, this suggests a successful cross-calibration of the reference and non-reference missions in our solution framework. The extension of our solution to use multi-mission data (i.e., reference and non-reference missions) advanced the approach in slightly improving the coherence of ASL trends at TGs (Figure S24), and decreasing the geographical variability of ASL trends by $\sim 13 \%$ (i.e., RMSE of $0.39 \mathrm{~mm} / \mathrm{yr}$ fitted to the quadratic polynomial compared to $0.45 \mathrm{~mm} / \mathrm{yr}$ from the reference-mission solution, Figure S25). This experiment also reveals that the multi-mission solution outperformed the reference-mission implementation in capturing the variability in non-linear VLMs at TGs (with STD of the stacked non-linear series of $1.58 \mathrm{~mm}$ versus $1.42 \mathrm{~mm}$ from the reference-mission solution, closer to the 
STD of $1.59 \mathrm{~mm}$ from the benchmark raw GPS stack, with an increase in the respective correlation from +0.22 to +0.34 (see Figure S12)). We return to this comparison when evaluating the noise magnitudes of ATG combinations from reference and non-reference constellations (see Section 4.6).

Second, we evaluated the impact of estimating the temporal evolution in bias drifts on the stacked non-linear VLMs at TGs. We estimated the stack of varying TG VLMs (with the same process noise) where the systematic errors in multi-mission altimeters were either treated as linear quantities or were left to evolve non-linearly with time. The solution with the linear treatment of drift estimates adversely affected the common-mode variability of non-linear TG VLMs. As such, the STD of the stacked non-linear VLMs at TGs was increased to $2.54 \mathrm{~mm}$, which is less consistent with the independent stack of non-linear VLMs at GPS sites (Figure S13). This supported the possibility of time-variable behaviour in the mission-specific bias drifts, especially in the first $\sim 3.5$ years of Jason-2 operation. This experiment also showed a good consistency between the most probable estimates of time-fixed and time-variable bias drifts across each mission (compare Figure 5 with Figure S16), with an averaged difference of $-0.05 \mathrm{~mm} / \mathrm{yr}$ and STD of $0.25 \mathrm{~mm} / \mathrm{yr}$. This internal agreement added further confidence to our interpretation regarding the likely presence of non-zero systematic errors in the altimetry datasets in this region.

Third, we evaluated the effect of considering temporal evolution in TG VLMs on resolving the bias drifts. We ran the multi-stage solution approach for an experiment where non-linear components of VLM were constrained to be zero at all land-based geodetic sites, and the linear VLMs were constrained to the same estimates as the preferred solution (Figure 2a). This assumption led to small shifts relative to the preferred solution in the averaged magnitudes of timevariable bias drift estimates (from $-0.73 \mathrm{~mm} / \mathrm{yr}$ for SARAL to $+0.89 \mathrm{~mm} / \mathrm{yr}$ for TOPEX-side B). We could however discern a substantial difference in the pattern of the bias drift for the Jason-2 mission with a clear exacerbation of the anomaly around 2011 (compare Figures 5 and S17). Iterating this experiment with the reference-mission-only data yielded approximately the same findings (see Figure S18). These results suggest the important role of appropriate tuning to balance the differentiation between estimates of the common-mode TG loadings and the time variability of bias drifts. 
We further repeated the investigation described above but assumed the bias drifts behave linearly in time (Figures S19 and S20 in the cases of multi-mission and reference-mission solutions, respectively). This yielded comparable results for the bias drifts with a weighted average discrepancy of -0.12 and the STD of $0.43 \mathrm{~mm} / \mathrm{yr}$, confirming the estimated magnitudes in the region were internally consistent (compare Figures S17 and S19). We also tested small changes to the process noise of non-linear VLMs to assess the sensitivity of our estimate of loading deformation at TGs. These results support the suitability of our selected process noise to appropriately capture the stacked non-linear VLM in the preferred solution (Figure S11). Collectively, these findings are of significance to the altimetry community, suggesting some residual issues in the quality of the first $\sim 3.5$ years of the Jason-2 orbit solution (in this case, the CNES-GDRE product) over the Australian region.

Lastly, we assessed the impact of considering mission-specific bias drifts on our estimates of the linear TG VLMs, ASL rates, and non-linear TG VLMs around the region. We ran our multistage solutions with the zero-drift assumption across all missions. The linear VLM estimates showed marginally greater scatter $(0.64$ versus $0.56 \mathrm{~mm} / \mathrm{yr})$, per our preferred solution. The averaged ASL rate is slightly underestimated by $\sim 0.08 \mathrm{~mm} / \mathrm{yr}$ around the region if the bias drifts were discarded $(+3.32 \pm 0.38 \mathrm{~mm} / \mathrm{yr}$ versus $+3.40 \pm 0.34 \mathrm{~mm} / \mathrm{yr}$ from the solution when the bias drifts were applied), suggesting a negligible effect on monitoring the regional sea-level rise (Figure S26). This assumption also led to an increase in the variability of stacked non-linear TG VLMs with a STD of $2.16 \mathrm{~mm}$, relatively higher than that of the GPS-derived stack (compare Figure 4 with Figure S14). This was expected given the non-linear behaviour of Jason-2 drift in the period of 2010-12.

\subsection{Residuals and a posteriori analysis}

Our framework offered several opportunities to check how well the filter works to capture parameters that evolve across the time and space domains. We first monitored the temporal convergence between a posteriori estimates of filtered and smoothed solutions for each set of sitespecific parameters in the state vector: intercept, ASL slope, linear VLM, and non-linear VLM, at each different stage of the multi-stage approach (see Figures S27-S30 for an example of each 
parameter for a representative $\mathrm{CP}$ and $\mathrm{TG}$ pair). Second, we evaluated a posteriori estimates of averaged "white plus AR1" ATG noise (on a per TG basis) across all missions in the subsequent solutions (see Figures S31 and S32 for an illustrative example). Third, we investigated the spatial variability in the derived across-track ASL slopes, with respect to the a priori values (Figure S33). These warn of anomalous cases that likely have inappropriate settings for the state process noise, which fails to enable the decorrelation of signals and noise at specific sites.

To ensure the discrepancy in the a priori and a posteriori estimates of VLM "datum" does not exceed our average uncertainty (i.e., $\pm 0.10 \mathrm{~mm} / \mathrm{yr}$ derived nominally as the standard error from GPS-Krig uncertainties), we compared the weighted-average differences between our multimission TG VLM trends and the GPS-Krig interpolations (Figure 3). We further computed the weighted average of our a posteriori estimates of linear TG VLMs per cycle and monitored the stability over time to ensure there was no spatially-correlated common-mode variability reflected in the VLM datum; that otherwise would lead to imperfect estimates of mission-specific bias drift (see Figure S34). This analysis further supports the high bias drift for the TOPEX-side B mission in the study region.

To trace the fingerprint of residual oceanographic signals, we computed the noise magnitudes of ATG combinations from the last step of the multi-stage solution, between each pair of TG and CP pertaining to the Jason-series, Envisat-series and Sentinel-3A constellations. Figure 9 shows the results as a function of distances between CPs and the TGs and the coast, as well as whether the CPs were 'on' or 'off' the continental shelf, around the region. This revealed that the ATG noise magnitudes are generally increased as a function of CP distance from either TGs or the coast, though the relationship becomes more pronounced as a function of the CP distances off the coast. Unsurprisingly, ATG observations with the highest noise magnitudes were often associated with CPs located off the continental shelf where ocean dynamics are likely to differ considerably from those at the TG locations. 


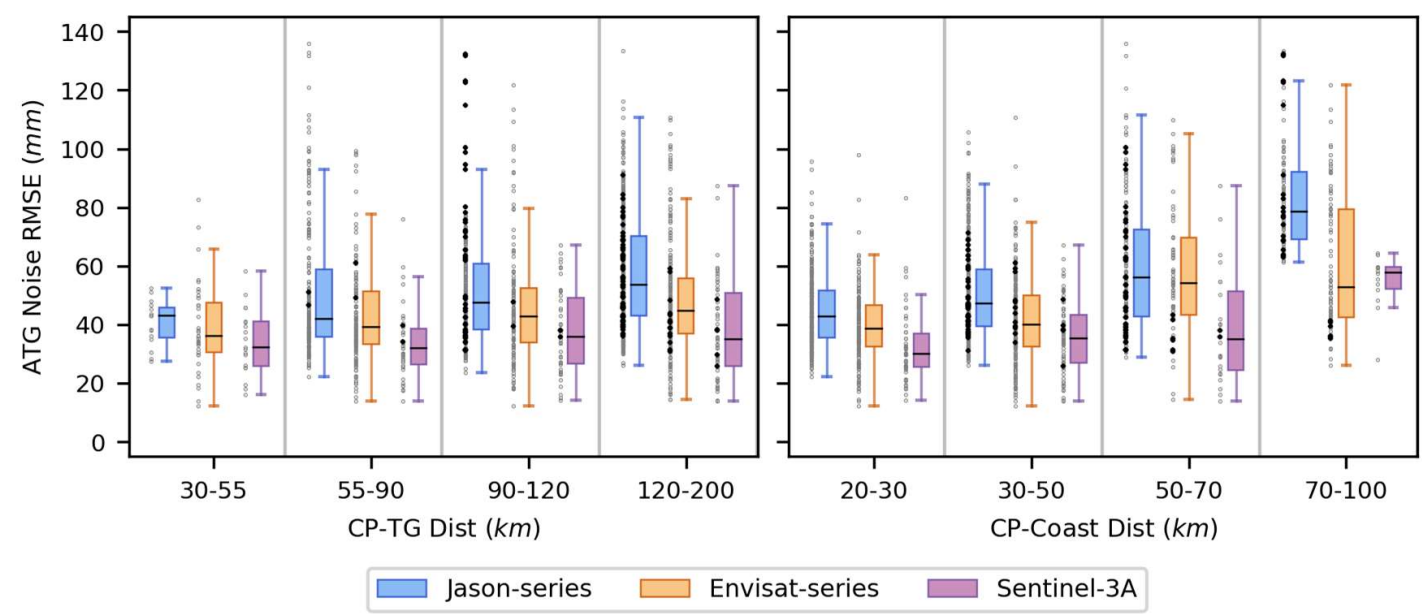

Figure 9. The "white plus AR1" noise magnitudes of altimeter minus tide gauge (ATG) series from the last solution of our multi-stage approach as a function of distances of comparison points (CPs) from (left) TGs and (right) coast, using the Jason-series, Envisat-series, and Sentinel-3A constellations. Note an intrinsic increase in the noise level as a function of the distances, though this pattern is more evident in the distances to the coast compared to the distances to TGs. CPs located "on" and "off" the continental shelf are differentiated with cyan and black dots, respectively.

We sought to further investigate the regional pattern of noise magnitudes on a per TG basis. As shown in Figure 10, we observed highest noise amplitudes at TGs in the North and North-West (i.e., BOOB, DARW and BROO). These sites showed high correlation between noise magnitude and distance between $\mathrm{CP}$ and the coast (rather than distance to TG). We note both distance metrics are influenced by coastal geometry and geometry of satellite ground tracks. We also noticed a relatively lower median level of ATG noise for the non-reference missions, compared to those pertaining to the reference missions. Given the ground track pattern, it follows that the median distances of the non-reference CPs to the coast were slightly less than those from the Jason-series missions (with the highest values in the North and North-West regions, related to the coastal geometry of these areas).

To understand the potential impact of residual oceanographic signals on our estimates of localized non-linear variability, we compared the spatial coherence of the dispersion in non-linear TG VLMs with the ATG noise magnitudes at individual gauges ordered anti-clockwise around Australia commencing in the North-East (top panel, Figure 10). This revealed that the ATG noise magnitudes are closely correlated with the amplitudes of non-linear VLMs, with highest values for 
both in the North and North-West (i.e., TGs at BOOB, DARW and BROO locations). The magnitude of VLM variability also showed some correlation with distance from CP to the coast, suggesting the potential for CPs to be sampling different oceanographic regimes, especially in areas where the continental shelf is narrow and thus influencing the results. Sites with higher amplitudes of non-linear VLM typically had higher distances between CP and the coast (compare top and middle panels, Figure 10) with less discernible correlation with distances between CP and the TG locations.
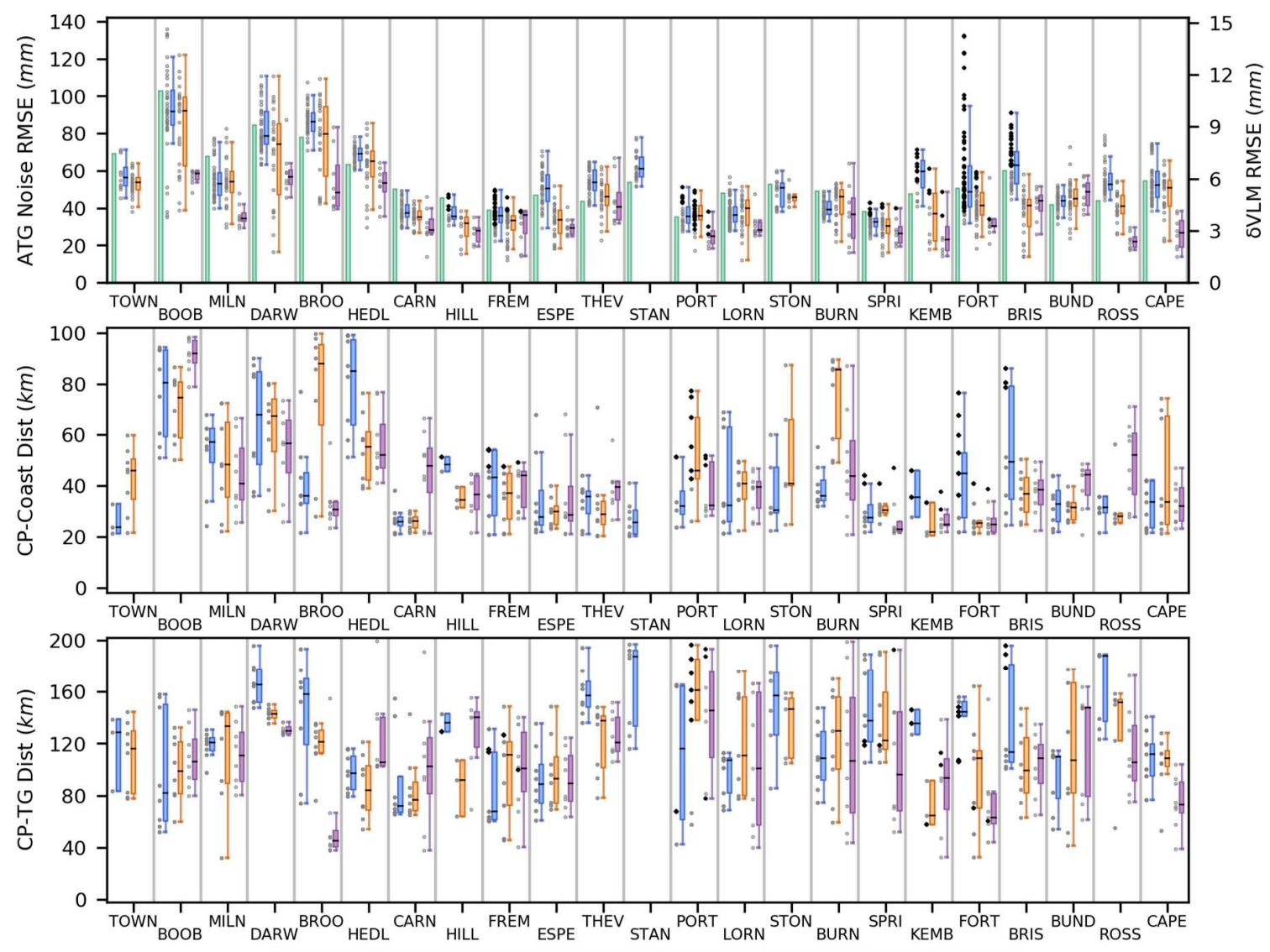

$\square$ OVLM $\square$ Jason-series $\square$ Envisat-series $\square$ Sentinel-3A

Figure 10. Spatial variability in (top) the root mean squared error (RMSE) of non-linear vertical land motion (green bars, $\delta \mathrm{VLM}$, right-hand axis) and "white plus AR1" noise of altimeter minus tide gauge (ATG, left-hand axis) observations, (middle) distances between comparison points (CPs) and the coast, and (bottom) separations of CPs from TGs, pertaining to the Jason-series (blue), Envisat-series (orange) and Sentinel-3A (purple) constellations on a per TG basis. Note different scales on y-axes. CPs located "on" and "off" the continental shelf are differentiated with cyan and black dots. TGs are ordered anti-clockwise around Australia, commencing with TOWN located on the North-East coast. 
Differentiating out CPs based on their location with respect to the continental shelf was informative. We found that $\sim 15.2 \%, 6.7 \%$ and $6.2 \%$ of our ATG observations were formed using off-shelf CPs pertaining to the Jason-series, Envisat-series and Sentinel-3A constellations, respectively. In most cases, CPs located 'off' the shelf had significantly greater noise (top panel, Figure 10). We computed the ATG noise magnitudes pertaining to the TGs adjacent to regions with the narrowest shelf width (HILL, FREM, PORT, SPRI, KEMB, FORT and BRIS locations) separately using the CPs that are situated 'on' or 'off' the narrow stretches of shelf. As shown in Figure 11, the ATG noise at these gauges are noticeably increased as the CPs are located off the shelf, especially in the case of Jason-series combinations, and particularly with respect to the FORT gauge. To further investigate the potential impact of potential residual trends in ATG series caused by differential oceanographic signals, we separately compared the weighted average linear VLMs from the ATG combinations associated with their respective on-shelf and off-shelf CPs (Figure 11). This comparison suggests the presence of non-zero residual trends of up to $\sim \pm 0.5$ $\mathrm{mm} / \mathrm{yr}$ in the ATG differences, likely due to different oceanographic regimes between these gauges and the CPs on or off the shelf. We return to this issue in the discussion.

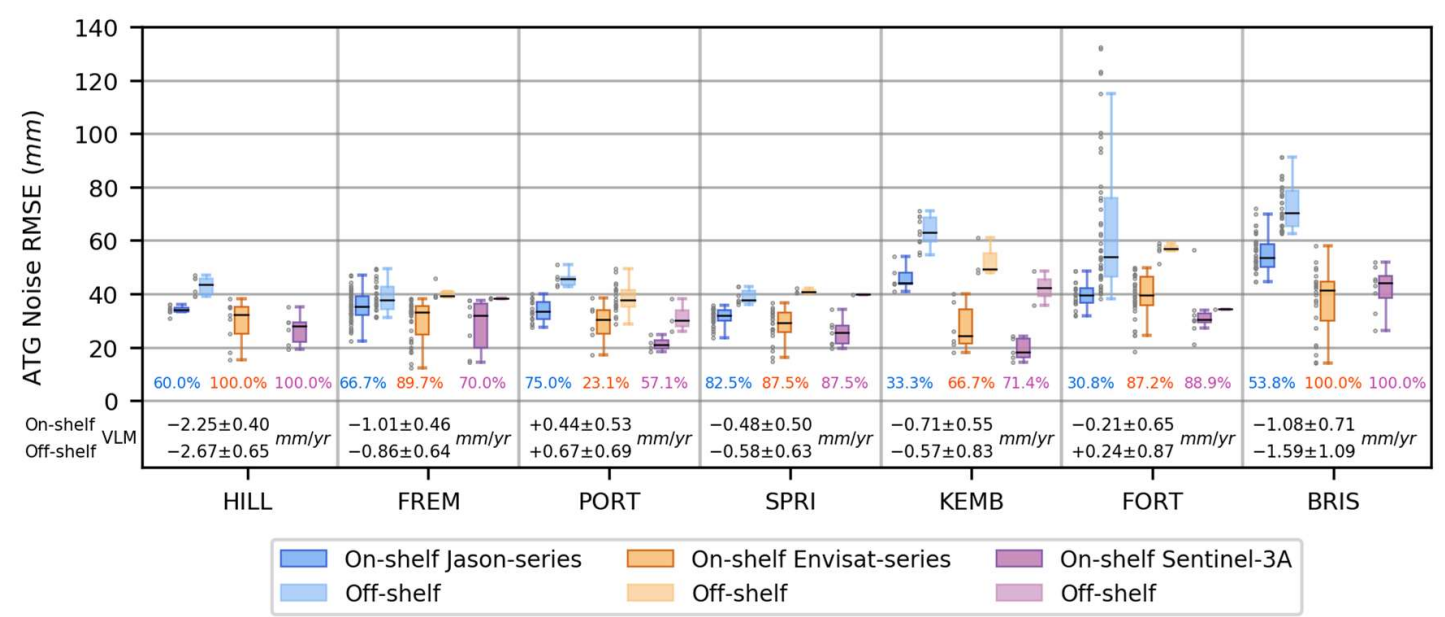

Figure 11. The "white plus AR1" noise of altimeter minus tide gauge (ATG) series, pertaining to the Jasonseries, Envisat-series and Sentinel-3A constellations associated with the TGs subject to the narrow stretches of shelf. Note the annotated percentage of the ATG observations associated with CPs that are located well over the continental shelf. Also note the weighted average of TG VLMs annotated, that were computed using the ATG differences associated with their respective on-shelf and off-shelf CPs. 
Finally, we checked the weighted average stack of the "white plus AR1" noise estimates of the ATG combinations to assess the presence of any uncaptured trends or time-varying signals over the region (Figure 12). As expected, the RMSE values over our domain were marginally higher than those from global studies (e.g., Watson et al., 2015), yet comparable with findings over the Baltic Sea region by Rezvani et al. (2021). This investigation reveals that negligible signal remains unmodelled in the ATG residuals, which supports the validity of our intrinsic assumptions and that our filter was tuned appropriately. We refer the readers to Rezvani et al. (2021) for further information about the tuning process and performance evaluations.
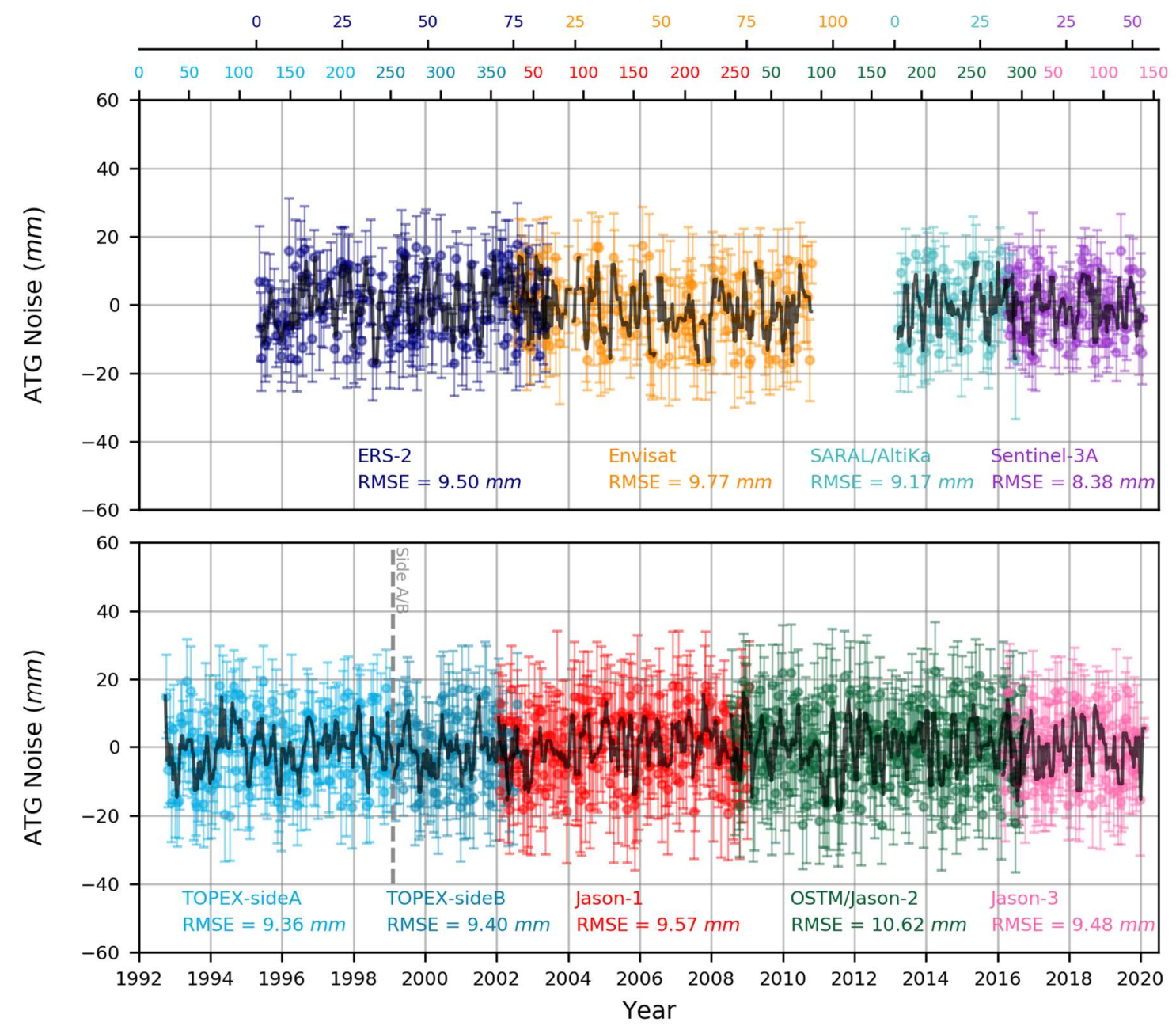

Figure 12. Cycle-by-cycle weighted average stack of "white plus AR1" residuals of altimeter minus tide gauge (ATG) formations in the region, inferred from the last solution of our multi-stage solution approach, with root mean squared error (RMSE) of mission-specific noise annotated. The observational cycles of nonreference and reference satellites are shown on the top axes with the same colours. 


\section{Discussion}

We have investigated time-fixed and time-variable components of site-specific VLMs and mission-specific systematic errors around the Australian continent using a novel analysis of altimetry, GPS, and TG records. A key assumption of our approach was that linear VLMs from GPS sites, spatially interpolated to TG locations, were sufficiently accurate throughout the network to stabilize an a priori VLM "datum" for the initial determination of systematic errors in altimeter datasets. As noted by Rezvani et al. (2021), a network-wide mean error in a priori VLM has the potential to map into the average estimates of altimeter bias drift. The validity of this assumption was strengthened with the inclusion of a significant number of geodetic sites around the Australian region. A further assumption was that the multi-stage solutions could adequately separate what are otherwise highly-correlated parameters. We attempted to achieve this by gradually differentiating the state components, making use of a suitable set of constraints in different parts of the engine, taking advantage of a priori knowledge about the underlying unknowns and noise characteristics.

Our approach confirms the inadequacy of GIA alone (defined here by the ICE-6G_D model) to represent VLM around the continental Australia. Our VLM estimates at both GPS and TG sites showed a widespread pattern of subsidence, with the respective median trends of -0.55 and -0.66 $\mathrm{mm} / \mathrm{yr}$. Comparing these with the GIA-inferred median suggested that the ICE6G_D predictions generally underestimate the present-day subsidence of the continent by $\sim 0.45 \mathrm{~mm} / \mathrm{yr}$, though this discrepancy is partly explained by geo-centre motions in the vertical direction (e.g., Sun \& Riva, 2020). Our findings are broadly consistent with the results from a more detailed assessment of VLM using a different and more comprehensive network of GPS sites by Riddell et al. (2020), highlighting the deficiency of GIA alone in the context of inferring VLM for regional sea-level studies. We note that Riddell et al. (2020) was unable to attribute the observed subsidence to a known geophysical source, but that Riddell et al. (2021) suggest the subsidence may have postseismic origins since 2004.

We detected localized VLM trends at some TGs that are significantly different to the GPSinterpolated values. Comparing the estimates of linear VLMs at TGs with the nearby GPS sites within $15 \mathrm{~km}$ revealed noticeable differences at very local scales, yet a negligible weighted average difference of $-0.12 \mathrm{~mm} / \mathrm{yr}$. The variability in these differences was quite high, with the STD of $0.81 \mathrm{~mm} / \mathrm{yr}$ and WRMSE of $0.73 \mathrm{~mm} / \mathrm{yr}$. Note, however, the dissimilarity between timespans of 
the TG and GPS records as listed in Tables S4 and S5, respectively. These suggest that we detected possible spatial variability in time-fixed components of VLM, calling into question the adequacy of GPS VLM Kriging (of comparatively short records) to TG locations in the region. As with previous studies, this work remains predicated on the assumption that there is no significant trend in the difference of absolute sea-level between TG and ALT (CP) measurement locations. The alternate hypothesis is that the ASL trends at TG and CP locations may be different due to the physical processes affecting sea-level variability driven by potential trends in local-scale influences such as wind stress, river runoff, and coastal-trapped propagations as well as remote effects of the large-scale ocean circulation (e.g., Ponte et al., 2019). Despite the siting of the chosen TGs mostly in locations thought to be well connected to the open ocean, residual trends in ocean signals may at least partially contribute to what we inferred as localized VLM trends at some TGs. This is especially the case in areas with regions with narrow shelf widths where some CP locations were not adequately located on the shelf, and thus likely sampling a different oceanographic regime (Figure 11). We note however that the increased noise associated with such CPs is inherently incorporated into the Kalman engine, hence these CPs will have less influence than others located on the shelf with reduced noise amplitude.

Our VLM trends, applied to the RSL records, generally improved (as expected) the spatial coherence in ASL rates at TGs around the study region. The scatter of our TG ASLs was 0.56 $\mathrm{mm} / \mathrm{yr}$, significantly smaller than when GPS-Krig $(0.94 \mathrm{~mm} / \mathrm{yr})$ or GIA $(0.97 \mathrm{~mm} / \mathrm{yr})$ were used. Also, our scatter of ASL estimates was slightly better than the solution when bias drifts were constrained to be zero across the satellite era $(0.56 \mathrm{vs} .0 .64 \mathrm{~mm} / \mathrm{yr})$. In a regional context, the scatter of our ASL estimates was $0.52,0.43,0.29$, and $0.15 \mathrm{~mm} / \mathrm{yr}$ in the NW, SE, NE, and SW of Australia, respectively. Compared to the spatial pattern described by White et al. (2014), these estimates show a reduction in ASL variability around the coast, likely driven by the 10 years increase in the timescale studied.

We estimated an average rate of ASL rise at TGs to be $+3.40 \pm 0.34 \mathrm{~mm} / \mathrm{yr}$, which is well supported (as expected) by the average estimate of $+3.51 \pm 0.26 \mathrm{~mm} / \mathrm{yr}$ from ALT records after the time-variable drifts (Figure 5) as well as relative intra- and inter-mission biases (Figures S21 and S22) applied. There appeared to be a slight (but insignificant) SE-NW gradient in ASL trends $(+3.24 \pm 0.33$ to $+3.83 \pm 0.69 \mathrm{~mm} / \mathrm{yr})$ with the North and North-West areas exposed to the higher 
rates, potentially in response to the ENSO-related effects (White et al., 2014). Having adjusted for the effect of changing ocean volume caused by GIA (Peltier 2004), our average rates of sea-level rise increase to $+3.80 \pm 0.34$ and $+3.87 \pm 0.23 \mathrm{~mm} / \mathrm{yr}$ for TG and ALT records respectively, which are $\sim 1.0 \mathrm{~mm} / \mathrm{yr}$ higher than that from White et al. (2014) who used data up to the end of 2010 . Our use of an additional 10 years of data suggests an acceleration in sea-level around Australia, consistent with findings across the Oceania region from Wang et al. (2021). Our adjusted ASL estimates also appear marginally higher than the global rate of sea-level rise $(+3.40 \pm 0.22 \mathrm{~mm} / \mathrm{yr}$, updated from Beckley et al., 2017).

Small systematic errors in altimetry may hinder attempts to accurately monitor sea-level changes at regional scales (e.g., Ablain et al., 2015). We estimated mission-specific errors that were typically non-zero in a regional context, that were cohesive in a multi-mission sense and had some time-variability (Figure 5). The weighted average of the evolving systematic bias for each mission ranged from $-1.09 \mathrm{~mm} / \mathrm{yr}$ for Jason- 1 to $+4.80 \mathrm{~mm} / \mathrm{yr}$ for TOPEX-side B, and typically converged after $\sim 2.5$ years. The OSTM/Jason-2 drift behaved anomalously particularly in the first $\sim 3.5$ years of the operation with the most dynamic component corresponding to the time of an exceptional La Niña in 2010-2012. Our further investigation showed that similar patterns were derived for time-varying bias drifts of the reference missions when the non-reference datasets were excluded (compare Figure 5 with Figure S15). Our analysis of a posteriori estimates of averaged "white plus AR1" ATG noise (on a per TG basis, Section 4.6) tends to support the conclusion that the significant time-variability of Jason-2 systematic errors is highly unlikely to be driven by spurious TG records.

The time variable behaviour of Jason-2 systematic error is broadly consistent with results from in situ instrumentation at the Bass Strait altimeter validation facility (Watson et al., 2020), where a similar signal was observed and remained unexplained (noting the GPS record confirmed it was not associated with continental water loading of the crust). Beckley et al. (2012) reported a similar feature in an analysis that prompted improved time-variable gravity field modelling used in the process of precise orbit determination for the Jason-2 mission. Whether the atypically dominant 2010-12 La Niña (see Fasullo et al., 2013) was inadequately modelled by the low degree and order time-variable gravity field used in orbit determination, or whether there was for example, a dominant steric change between TG and CPs driven by enhanced continental water runoff, remains 
to be determined. There were no other comparably large ENSO events (which involved a constructive alignment of various modes of climate that influence Australian TWS) over the record to enable further comparison or investigation. The Envisat mission had also finished by this time preventing further cross calibration to isolate the cause.

Our estimated magnitudes of the reference-mission bias drifts in the Australian region were $-0.83 \pm 0.15,+4.80 \pm 0.26,-1.09 \pm 0.14,-0.50 \pm 0.16$ and $-0.55 \pm 0.18 \mathrm{~mm} / \mathrm{yr}$, compared to $+0.38 \pm 0.16$, $-4.62 \pm 0.36,-2.69 \pm 0.16,+2.60 \pm 0.13$ and $+0.70 \pm 0.32 \mathrm{~mm} / \mathrm{yr}$ for the Baltic region (Rezvani et al., 2021). Of interest, in the Australian analysis we observed the opposite sign for the TOPEX-side B and Jason-2 bias drifts compared to the Baltic region. Overall, however, the magnitudes of our regional systematic error estimates were consistent with the error budget assessments from differencing the leading orbit products (e.g., Belli et al., 2020; Couhert et al., 2015, 2018).

Datum instability of coastal gauges will impact trend analysis using RSL records (e.g., Nerem \& Mitchum, 2002; Watson et al., 2015; Woodworth et al., 2017). In our solutions, any undetected datum errors in RSL records could be misinterpreted as TG VLMs, yet given the number of gauges included, they will have negligible contribution to altimeter-specific bias drifts. Following the approach by Rezvani et al. (2021), we found that some ATG observations were likely to be contaminated with RSL datum errors (for instance, in case of STON TG during the operational span of Jason-2, Jason3, SARAL/AltiKa and Sentinel-3A missions as shown in Figure S4). These potentially outlying observations have been excluded from our final solution. The remaining records may still be influenced by small datum shifts that below our detection resolution (Rezvani et al., 2021), however these unavoidable effects on the bias drift estimates would be effectively mitigated with inclusion of time-varying TG VLMs in our framework which is an advance of our recently developed approach.

When investigating our underlying assumptions as well as noise in the ATG residuals, the oceanic context is important. Australia's east coast is noticeably dominated by the energetic western boundary system, the East Australian Current (EAC, e.g., Cetina-Heredia et al., 2014; Ridgway \& Hill, 2009). The EAC flows southward interacting with bathymetry and water masses well offshore, as well as moving on and off the continental shelf cyclically with likely impact on the narrow shelf circulation (Archer et al., 2017). The extension of the EAC $\left(\sim 31-33^{\circ} \mathrm{S}\right)$ has strengthened and extended further southward along the south-eastern Australia coast, becoming 
warmer and saltier, leading to higher-than-average rates of sea-level rise well off the shelf. The extent to which these highly-variable ocean-dynamics influence gradients across the narrow shelf, particularly close to the coast, is uncertain. Such gradients across all shelf areas around Australia have the potential to contribute to residual trends in the ATG series used here.

To mitigate the influence of dynamic changes in regions such as the EAC, and potential effects of gradients across the continental shelf, we have selected altimetry CPs to be within $20-120 \mathrm{~km}$ of the Australian coast. Given the average width of the shelf along the east coast is $\sim 25 \mathrm{~km}$ (Cresswell et al., 2017), we note that $\sim 15.2 \%, 6.7 \%$ and $6.2 \%$ of our ATG observations were formed using CPs located off the shelf, pertaining to the Jason-series, Envisat-series and Sentinel3A constellations, respectively. Unsurprisingly, our analysis shows increased ATG noise as the CP distances from the coast or TGs increased, with the highest magnitudes often associated with the off-shelf CPs where oceanic signals are likely to be substantially different from at the TG locations (Figures 9-11). Our investigation further reveals the largest noise magnitudes for TGs installed in the high-latitude regions that are more exposed to the ENSO-induced climate variability - this is likely related to the effects of complex bathymetry/geometry between the TG and CP as well (consistent with noise analysis by Burgette et al., 2013 and White et al., 2014). Owing to the effects of improved spatial sampling based on adding the non-reference missions, the multi-mission solution offered a potential way of combating this issue. Of interest, it was noted that the multi-mission derived VLM at TGs showed more comparable spatial variability as a function of inter-site spacing as expressed in the independent VLM record at GPS sites, than did the TG VLMs derived from the reference-mission solution (Figure S9; see also Santamaría-Gómez et al., 2017 for a more comprehensive analysis of intra-network differences in GPS VLM). Our approach could be further improved to remove the harmonic ocean tides at these locations given we only considered the standard constituents (including M4) in our analysis. The effects of internal tides were also not considered yet would likely contribute to the ATG noise in some regions around Australia (particularly at some CPs located offshore the NW coast).

Adjacent to the EAC, the FORT gauge is an interesting example for a TG which is not well connected to open ocean and sits adjacent to the narrow shelf in close proximity to the intensifying EAC (e.g., Johnson et al., 2011; Suthers et al., 2011). Given the geometry of the reference-mission ground tracks with respect to the coast and TG location, all reference-mission CPs were tightly 
clustered in terms of distance to the TG (Figure 10, bottom), yet variable in terms of distance to the coast (Figure 10, middle). The ATG noise magnitudes for this gauge and reference-mission data showed significant variation with distance from the coast (Figure 10, top), noting the greater noise for those CPs located off the shelf (Figure 11). Conversely, the geometry of the non-reference mission sampling enables lower separation distances and reduced variability (Figures 10 and 11). This suggests the impact of differential oceanographic signals, possibly related to the EAC extending onto the shelf. For this gauge, $\sim 69.2 \%$ and $\sim 12.8 \%$ of ATG observations are formed with CPs located off the shelf pertaining to the Jason-series and Envisat series, respectively (Figure 11). Given the reduced noise of on-shelf CPs, this highlights the benefit of including all missions in a single solution (Figure 9). Comparing the VLM trends for FORT TG using solely the on-shore and off-shore combinations further reveals the likely effect of sampling biases given a VLM trend difference of $\sim 0.5 \mathrm{~mm} / \mathrm{yr}$ (Figure 11).

Given the extended altimetry dataset, we found that the multi-mission framework generally provides more precise estimates of time-fixed and time-variable components of TG-specific VLMs with a $\sim 35 \%$ reduction in the formal errors (with significant differences in TG VLM from the reference-mission solution at higher latitudes, Figure S10). We thus expected VLM at TGs to be more consistent to the nearby GPS sites, as the effect of any trend in ATG differences driven by ocean signals would be less when the multi-mission ground tracks get closer to the TG locations (compare STD of $0.74 \mathrm{~mm} / \mathrm{yr}$ versus $0.83 \mathrm{~mm} / \mathrm{yr}$ for the VLM differences of multi-mission and reference-mission solutions, respectively, each compared to GPS-Krig at TG locations). The multimission estimates of linear VLM also resulted in a $\sim 13 \%$ decrease in the latitudinal-dependence of variability in the ASL trends at TGs (RMSE of $0.39 \mathrm{~mm} / \mathrm{yr}$ versus $0.45 \mathrm{~mm} / \mathrm{yr}$ from the multimission and reference-mission solutions, respectively). The averaged ASL rise in the region was estimated to be $+3.41 \pm 0.38 \mathrm{~mm} / \mathrm{yr}$ using the reference-mission data, approximately equivalent to the $+3.40 \pm 0.34 \mathrm{~mm} / \mathrm{yr}$ from the multi-mission solution (note the latter has slightly smaller uncertainty). The multi-mission approach yielded a $\sim 54 \%$ increase in the correlation coefficient between the non-linear VLM stacks at TGs and GPS sites, compared to the reference-mission solution. The multi-mission combination mitigated the impact of poor performance of any one mission, and indeed assisted the cross-calibration process in deriving consistent estimates of bias drift for the reference and non-reference missions. This could further assist improving our knowledge about long-term sea-level variability at regional scales. 
The limitation of the unknown contribution of differential oceanographic signals to the trend in ASL between the TG and offshore ALT (CP) locations was partially investigated by assessing the improvements gained when using non-reference mission data with typically improved spatial sampling closer to the gauge locations and with higher percentage of CPs located well on the shelf. Regardless of the advantages of multi-mission solution, in many cases we continue to lack ALT data adjacent to the TG locations (hence coastal retracking has only limited benefit), returning us to the vexing question of sampling the same ocean signals. In a broader context, the spatial coherence of the variability in the ATG noise and the non-linear TG VLMs (Figure 10, top) further suggests that the approach is not able to capture subtle geophysical signals such as the far-field post-seismic relaxation of the NW coast identified by Riddell et al. (2021). Further, the ATG observations formed with off-shelf CPs notably increased the noise magnitudes and likely included the non-zero residual trends within the range of $\sim \pm 0.1-0.5 \mathrm{~mm} / \mathrm{yr}$, which may bias our estimates of VLM at the respective gauges (Figure 11). The open question of the magnitude and spatial scale of differences in sea-level trends between the TG and ALT locations well on the shelf is a hard limit on the utility of all ATG-type techniques in fully resolving site-specific VLM and its evolution, especially in regions of complex geometry, narrow shelves, and dynamic oceanic conditions.

Like all Kalman-type engines, our approach requires appropriate settings and tuning for measurement noise and random-walk process noise. These were defined within the context of the study region and our a priori assumptions. In the initial work by Rezvani et al. (2021), bias drifts were resolved with tight constraints on TGs clearly exhibiting linear VLM, and loose constraints on TGs where substantial non-linearity existed. This differentiation in constraints was based on visual inspection and thresholding of the adaptive process noise and depended on variability in the regional velocity field. In this study, we considered a more flexible functional model for VLM, such that linear and non-linear variables were separately involved. This configuration is likely to improve the estimates of bias drift, despite the potential for small unresolved datum shifts in specific TG records (below the resolution of our detection strategy, Rezvani et al., 2021). Further, the estimates of bias drift could possibly be less affected by residual oceanographic signals between TG and ALT sample locations (as opposed to TG VLM). Our noise analysis tended to support this, such that the stacked ATG residuals over the region are not contaminated with any uncaptured trends or time-varying signals. Interestingly, the stacked residuals of Jason-2 and 
Sentinel-3A combinations are characterized with the highest and lowest RMSE, respectively (Figure 12). Overall, the enhancements presented here potentially makes the technique applicable to tectonically highly dynamic areas exposed to abrupt changes in VLM signals either due to sudden ice-mass loss or large earthquakes.

\section{Conclusions}

We further developed a Kalman-based methodology to simultaneously estimate site-specific VLM and altimeter-specific systematic errors using observational series of ALT minus TG, tandem/dual crossovers, and GPS heights. We used a multi-stage solution approach to cope with singularity of the underlying problem, such that the highly correlated unknowns were gradually separated in the presence of noise across space and time. We differentiated VLM parameters into linear and non-linear components to evaluate evolution in crustal motion at geodetic sites, and its impact on our ability to resolve time-variability in altimeter systematic errors. The presented method advances the ATG technique by 1) assimilating multi-mission records; and 2) exploring non-linearity in both altimeter drift and VLM terms.

Owing to the temporal and spatial limitations of GPS records and the fact that GIA models only reflect one driver of VLM, our approach offered the potential improvement of monitoring VLM and its variability at TG locations around continental Australia since the early 1990s. Our estimates of linear TG VLMs revealed widespread subsidence, with a maximum of $\sim-0.8,-1.8,-2.4$ and $-1.2 \mathrm{~mm} / \mathrm{yr}$ in the NW, NE, SW and SE sub-regions. Comparing to ICE6G_D model, the GIA rates in these sub-regions are lower by $\sim 0.5,2.0,0.9$ and $1.6 \mathrm{~mm} / \mathrm{yr}$, respectively, although these discrepancies are partly explained by the geo-centre movements in the polar direction (e.g., Sun \& Riva, 2020; Wu et al., 2012). We detected possible localized VLM trends at coastal TGs relative to the surrounding GPS bedrock velocities within $15 \mathrm{~km}$, with a negligible weighted average difference of $-0.12 \mathrm{~mm} / \mathrm{yr}$, but with quite high variability (STD of $0.81 \mathrm{~mm} / \mathrm{yr}$, and WRMSE of $0.73 \mathrm{~mm} / \mathrm{yr}$ ). This calls into question the adequacy of GPS VLM Kriging (of comparatively short records) to TG locations in the region, considering the often-untested assumption that there is no significant trend in the difference of absolute sea-level between TG and ALT (CP) measurement locations. 
The narrow continental shelf around Australia, in particular along the Eastern coast and its proximity to a dynamic and intensifying major boundary current system, provides the potential to further investigate the limitations of the method due mainly to the presence of residual oceanographic signals. These signals would originate from different local-scale and large-scale oceanic processes operating at the TG and CP locations, respectively. Our approach reveals the magnitudes of the ATG noise vary as a function of distances between TG and altimetry (CP) measurement locations, and in particular, from the coast. The highest noise magnitudes were often associated with CPs located off the continental shelf where significant residual signals of oceanic original likely exist between the TG and offshore ALT locations. Our study included $\sim 15.2 \%, 6.7 \%$ and $6.2 \%$ of the ATG observations formed using CPs being located off the shelf, pertaining to the Jason-series, Envisat-series and Sentinel-3A constellations, respectively. These observations pertained to the TGs at HILL, FREM, PORT, SPRI, KEMB, FORT and BRIS locations where the adjacent continental shelf is quite narrow. The ATG observations formed with off-shelf CPs are likely to bias the VLM estimates given the residual trends within the range of $\sim \pm 0.1-0.5 \mathrm{~mm} / \mathrm{yr}$.

Our solution notably detects a VLM anomaly of $\sim 2.0 \pm 0.72 \mathrm{~mm} / \mathrm{yr}$ between the FREM TG and the PERT GPS $\sim 31 \mathrm{~km}$ away in the Western Australia. For this gauge, we inferred that the $\sim 33.3 \%$ and $10.3 \%$ of the ATG observations formed using ALT data location off the shelf had little effect in biasing the estimated VLM rate. Also, an interesting (yet insignificant) VLM difference of $\sim 0.6 \pm 0.64 \mathrm{~mm} / \mathrm{yr}$ was found between the FORT TG and the FTDN GPS sites separated by $\sim 1 \mathrm{~km}$ in Eastern Australia, suggesting the possibility of a residual trend in the ATG series driven by the fact this gauge is not well connected to the open ocean and the ALT (CP) locations are often located off the narrow continental shelf subject to quite different ocean signals. Our investigation reveals that $\sim 69.2 \%$ and $\sim 12.8 \%$ of ATG observations for this gauge were formed using CPs located off the shelf pertaining to the Jason-series and Envisat series constellations, respectively. A comparison between the weighted average VLMs from on-shelf and off-shelf CPs further supports the potential presence of a residual trend of $\sim 0.5 \mathrm{~mm} / \mathrm{yr}$ in the ATG differences, highlighting the impact of different oceanographic signals at the off-shelf CPs in this region adjacent to a complex western boundary system.

Application of our time-fixed VLMs to RSL rates generally improved the spatial coherency in the resultant estimates of ASL trends at TGs, with a $\sim 42 \%$ and $\sim 48 \%$ reduction in the RMSE of a 
fitted quadratic polynomial per latitudes reduced to the SE-NW direction, compared to the GPSKrig and GIA alternatives, respectively. We derived an average ASL rate of $+3.40 \pm 0.34 \mathrm{~mm} / \mathrm{yr}$ from TG records using our VLM estimates, unsurprisingly in close agreement with the average estimate of $+3.51 \pm 0.26 \mathrm{~mm} / \mathrm{yr}$ from ALT records around the study region. A slight SE-NW gradient was evident in ASL trends $(+3.24 \pm 0.33$ to $+3.83 \pm 0.69 \mathrm{~mm} / \mathrm{yr})$, potentially driven by the ENSO effects (White et al., 2014). After adjusting the effect of GIA-induced ocean volume changes, our average rate of sea-level rise is noticeably higher than that from White et al. (2014), making use of an additional 10 years of data that suggests an acceleration in the sea-level changes around Australia, consistent with findings across the Oceania region from Wang et al. (2021).

The non-linear VLM stacks from TG and GPS showed some correlation, highlighting the method had some skill in capturing the common mode of deformation likely induced by surface loadings over the continent. However, the magnitude of residual ATG noise prevented the detection of small geophysical signals such as post-seismic relaxation along the NW Australian coast as identified by Riddell et al. (2021). The residual oceanographic signals between the TG and ALT CPs are likely the main contributing factor, especially for the ATG series pertaining to off-shelf CPs as well as TGs situated at higher latitudes (and particularly in geometrically complex areas). We inferred a similar spatial pattern of variability in both the estimates of ATG noise and non-linear TG VLMs, underscoring possible decorrelation issues due to these effects.

We detected significant altimeter-specific drifts (ranging from $-1.09 \mathrm{~mm} / \mathrm{yr}$ for Jason-1 to $+4.80 \mathrm{~mm} / \mathrm{yr}$ for TOPEX-side B) that are within the mission specifications and comparable to the rates observed from the differences in the leading orbit products (e.g., Couhert et al., 2015). Combined over the full altimetry era, these drifts had a negligible effect on linear rates of sea-level change (not considering them implied the underestimation of sea-level rise by $\sim 0.08 \mathrm{~mm} / \mathrm{yr}$ on average - this is unlikely to be statistically significant considering the errors involved). The altimeter drifts are likely to be spatially variable in the global context as indicated by Rezvani et al. (2021).

We identified an anomaly in the early period ( 2008.5-2012) of the Jason-2 mission performance. We excluded any potential artefact in the solution associated with the end of the Envisat mission as the partial cause of this as the signal was apparent in a reference-mission-only solution. A similar anomaly was observed and remained unexplained from in situ instrumentation 
at the Bass Strait altimeter validation facility (Watson et al., 2020). We speculated that this signal could be associated with inadequate representation of the anomalously large 2010-2012 La Niña event in the time-variable gravity field used for precise orbit determination (e.g., Beckley et al., 2012) or potential dominant steric changes driven by atypical continental runoff/discharge, yet it is impossible to dismiss other possible drivers (e.g., Belli et al., 2021; Couhert et al., 2018).

Limitations remain including the hard limit of variability (noise) and potential trends in differential oceanography as well as the inability to derive subtle non-linear signals as present in the Australian region. These emphasize the ongoing need to install GPS directly at the TG or nearest feasible locations (Woodworth et al., 2016). It is also important to further develop highresolution regional ocean models that resolve a full suite of coastal ocean processes (Ponte et al., 2019). Such models, however complex and as yet unavailable for Australian shelf waters, may offer the opportunity to further improve the ATG technique.

Our data-driven approach can be implemented in other study regions to evaluate the performance of the reference and non-reference altimetry systems in an integrated adjustment framework, leading to improved monitoring of regional sea-level changes. This method can be used to challenge the reliability of the often-made assumption of linear-only VLM, that would be beneficial for geophysical studies. This can also be used to examine the assumption of zero differential linear VLM between the TG and the nearby GPS sites. This approach assists in advancing our understanding of the impacts of climate change on sea-level variability at regional and global scales.

\section{Author contributions}

M.-H. Rezvani, C.S. Watson, and M.A. King designed research; M.-H. Rezvani and C.S. Watson performed research; M.-H. R. analysed data; M.-H. Rezvani, C.S. Watson, and M.A. King wrote the paper. 


\section{Acknowledgements}

This research was supported under the Australian Research Council's Special Research Initiative for Antarctic Gateway Partnership (Project ID SR140300001). Aspects of this work were also supported by the Australian Research Council Discovery Project DP150100615. This work has benefitted from the Bass Strait altimeter validation facility which is supported by Australia's Integrated Marine Observing System (IMOS) - IMOS is enabled by the National Collaborative Research Infrastructure Strategy (NCRIS). IMOS is operated by a consortium of institutions as an unincorporated joint venture, with the University of Tasmania as Lead Agent. Access to the RADS altimeter, local tide gauge and NGL databases are appreciated. We acknowledge the TPAC High Performance Computing facilities used for computations. We acknowledge discussions held with Benoit Legresy and Richard Coleman which were useful in preparing this manuscript.

\section{Data availability}

The altimeter, TG, and GPS data used in this study are publicly available through https://github.com/remkos/rads, http://www.bom.gov.au/metadata/catalogue/search.shtml?page=5, and http://geodesy.unr.edu/, respectively. Dynamic atmospheric Corrections are produced by CLS using the Mog2D model from Legos and distributed by Aviso+, with support from CNES (https://www.aviso.altimetry.fr/). The ICE6G_D GIA model is available through http://www.atmosp. physics.utoronto.ca/ peltier/data.php.

\section{References}

Altamimi, Z., Collilieux, X., \& Métivier, L. (2011). ITRF2008: an improved solution of the international terrestrial reference frame. Journal of Geodesy, 85, 457-473. https://doi.org /10.1007/s00190-011-0444-4

Archer, M. R., Roughan, M., Keating, S. R., \& Schaeffer, A. (2017). On the variability of the East Australian Current: Jet structure, meandering, and influence on shelf circulation. Journal of Geophysical Research: Oceans, 122, 8464-8481. https://doi.org/10.1002/2017JC013097

Beckley, B.D., Zelensky, N.P., Yang, X., Melachroinos, S., Chinn, D., Lemoine, F.G., Ray, R.D., Brown, S., \& Mitchum, G. (2012). Reassessment of Jason-2 stability based on revised POD standards. Ocean Surface Topography Science Team Meeting 2012. Retrieved from 
https://www.aviso.altimetry.fr/fileadmin/documents/OSTST/2012/oral/01 thursday 27/05 re gional_and_global_calval_II/06_CV2_Beckley.pdf

Belli A., Zelensky N. P., Lemoine F.G., Chinn D.S. (2021). Impact of Jason-2/T2L2 UltraStable-Oscillator Frequency Model on DORIS stations coordinates and Earth Orientation Parameters. Advances in Space Research. 67, 930-944. https://doi.org/10.1016/j.asr.2020.11. $\underline{034}$

Bevis, M., \& Brown, A. (2014). Trajectory models and reference frames for crustal motion geodesy, Journal of Geodesy, 88(3), 283-311. https://doi.org/10.1007/s00190-013-0685-5

Blewitt, G. (2003). Self-consistency in reference frames, geocenter definition, and surface loading of the solid Earth. Journal of Geophysical Research, 108(B2). https://doi.org/ $\underline{10.1029 / 2002 J B 002082}$

Boening, C., Willis, J. K., Landerer, F. W., Nerem, R. S., \& Fasullo, J. (2012). The 2011 La Niña: So strong, the oceans fell, Geophysical Research Letters, 39, L19602. https:// doi.org/10.1029/2012GL053055

Bos, M.S., Fernandes, R.M.S., Williams, S.D.P., \& Bastos, L. (2013). Fast Error Analysis of Continuous GNSS Observations with Missing Data. Journal of Geodesy, 87(4), 351-360. https://doi.org/10.1007/s00190-012-0605-0

Bouin, M.-N., \& Wöppelmann, G. (2010). Land motion estimates from GPS at tide gauges: A geophysical evaluation, Geophysical Journal International, 180(1), 193-209. https://doi.org/ 10.1111/j.1365-246X.2009.04411.x

Burgette, R.J., Watson C.S., Church J.A., White N.J., Tregoning P., \& Coleman R. (2013). Characterizing and minimizing the effects of noise in tide gauge time series: relative and geocentric sea level rise around Australia, Geophysical Journal International, 194(2), 719-736. https://oi.org/10.1093/gii/ggt131

Buttkus, B. (2000). Spectral analysis and filter theory in applied geophysics (1st ed.). SpringerVerlag Berlin Heidelberg. http://doi.org/10.1007/978-3-642-57016-2

Cetina-Heredia, P., M. Roughan, E. van Sebille, and M. A. Coleman (2014). Long-term trends in the East Australian Current separation latitude and eddy driven transport, Journal of Geophysical Research: Oceans, 119, 4351-4366, https://doi.org/10.1002/2014JC010071

Couhert, A., Cerri, L., Legeais, J.F., Ablain, M., Zelensky, N.P., Haines, B.J., Lemoine, F.G., Bertiger, W.I., Desai, S.D., \& Otten, M. (2015). Towards the $1 \mathrm{~mm} / \mathrm{y}$ stability of the radial orbit error at regional scales. Advances in Space Research, 55(1), 2-23. https://doi.org/10.1016 /j.asr.2014.06.041

Cresswell, G.R., Peterson, J.L., \& Pender, L.F. (2017). The East Australian Current, upwellings and downwellings off eastern-most Australia in summer. Marine and Freshwater Research 68(7), 1208-1223. https://doi.org/10.1071/MF16051

Dangendorf, S., Marcos, M., Müller, A., Zorita, E., Riva, R., Berk, K., \& Jensen, J. (2015). Detecting anthropogenic footprints in sea level rise. nature communications, 6, 7849. https://doi.org/10.1038/ncomms8849 
Fasullo, J. T., Boening, C., Landerer, F. W., \& Nerem, R. S. (2013). Australia's unique influence on global sea level in 2010-2011, Geophysical Research Letters, 40, 4368-4373. https://doi.org/10.1002/grl.50834

Featherstone, W. E., N. T. Penna, M. S.Filmer, and S. D. P. Williams (2015). Nonlinear subsidence at Fremantle, a long-recording tide gauge in the Southern Hemisphere, Journal of Geophysical Research: Oceans, 120, 7004-7014. https://doi.org/10.1002/2015JC011295

Grewal, M. S., \& Andrews, A. P. (2008). Kalman filtering: Theory and practice using MATLAB® (3rd ed.). WILEY. http://doi.org/10.1002/9780470377819

Hamlington, B.D., Thompson, P., Hammond, W.C., Blewitt, G., Ray, R.D. (2016). Assessing the impact of vertical land motion on twentieth century global mean sea level estimates, Journal of Geophysical Research: Oceans, 121, 4980-4993. http://doi.org/10.1002/2016JC011747

Hammond, W. C., Blewitt, G., Kreemer, C., \& Nerem, R. S. (2021). GPS Imaging of global vertical land motion for studies of sea level rise. Journal of Geophysical Research: Solid Earth, 126, e2021JB022355. https://doi.org/10.1029/2021JB022355

Han, S.-C. (2017). Elastic deformation of the Australian continent induced by seasonal water cycles and the 2010-2011 La Niña determined using GPS and GRACE, Geophysical Research Letters, 44, 2763-2772. https://doi.org/10.1002/2017GL072999

Holbrook N.J., Goodwin I.D., McGregor S., Molina E., Power S.B. (2011). ENSO to multidecadal time scale changes in East Australian Current transports and Fort Denison sea level: oceanic Rossby waves as the connecting mechanism. Deep-Sea Res. II Top. Stud. Oceanogr., 58(5), 547-558. https://doi.org/10.1016/j.dsr2.2010.06.007

Holbrook, N. J., Davidson, J., Feng, M., Hobday, A. J., Lough, J. M., McGregor, S., and Risbey, S. (2009). El Niño-Southern Oscillation. In 'Marine Climate Change in Australia: Impacts and Adaptation Responses 2009 Report Card'. NCCARF Publication 05/09. (Eds E. S. Poloczanska, A. J. Hobday and A. J. Richardson.) (National Climate Change Adaptation Research Facility.) Retrieved from http://www.oceanclimatechange.org.au/content/images/uploads/ENSO-final. pdf.

Johnson C.R., Banks S.C., Barrett N.S., Cazassus F., Dunstan P.K., Edgar G.J., Frusher S.D., Gardner C., Haddon M., Helidoniotis F., Hill K.L., Holbrook N.J. (2011). Climate change cascades: Shifts in oceanography, species' ranges and subtidal marine commu nity dynamics in eastern Tasmania. Journal of Experimental Marine Biology and Ecology, 400(1-2):17-32. https://doi.org/10.1016/j.jembe.2011.02.032

King, M.A., Keshin, M., Whitehouse, P.L., Thomas, I.D., Milne, G., \& Riva, R.E.M. (2012). Regional biases in absolute sea-level estimates from tide gauge data due to residual unmodeled vertical land movement. Geophysical Research Letters, 39(14), L14604. https://doi.org/10. $\underline{1029 / 2012 \mathrm{gl} 052348}$

Lyard, F.H., Allain, D.J., Cancet, M., Carrère, L., \& Picot, N. (2021). FES2014 global ocean tide atlas: Design and performance. Ocean Science, 17(3), 615-649. https://doi.org/10.5194/os$\underline{17-615-2021}$

Maronna, R.A., Martin, R.D., \& Yohai, V.J. (2006). Robust Statistics, Theory and Methods. New York: John Wiley \& Sons, Ltd. http://doi.org/10.1002/0470010940 
McGrath, G. S., Sadler, R., Fleming, K., Tregoning, P., Hinz, C., \& Veneklaas E. J. (2012). Tropical cyclones and the ecohydrology of Australia's recent continental-scale drought, Geophysical Research Letters, 39, L03404. http://doi.org/10.1029/2011GL050263

Nerem, R. S., \& Mitchum, G. T. (2002). Estimates of vertical crustal motion derived from differences of TOPEX/POSEIDON and tide gauge sea level measurements. Geophysical Research Letters, 29(19), 41-44. https://doi.org/10.1029/2002g1015037

Nerem, R. S., Beckley, B. D., Fasullo, J. T., Hamlington, B. D., Masters, D., \& Mitchum, G. T. (2018). Climate-change-driven accelerated sea-level rise detected in the altimeter era. Proceedings of the National Academy of Sciences of the United States of America, 115(9), 2022-2025. https://doi.org/10.1073/pnas.1717312115

Peltier, W.R. (2004). Global Glacial Isostasy and the Surface of the Ice-Age Earth: The ICE5G(VM2) model and GRACE. Annual Review of Earth and Planetary Sciences. 2004. 32,111149. https://doi.org/10.1146/annurev.earth.32.082503.144359

Peltier, W.R., Argus, D.F., \& Drummond, R. (2018). Comment on An Assessment of the ICE6G_C (VM5a) Glacial Isostatic Adjustment Model by Purcell et al. Journal of Geophysical Research: Solid Earth, 123(2), 2019-2028. https://doi.org/10.1002/2016JB013844

Pfeffer J., Allemand P. (2016). The key role of vertical land motions in coastal sea level variations: a global synthesis of multisatellite altimetry, tide gauge data and GPS measurements, Earth and Planetary Science Letters, 439, 39-47. https://doi.org/10.1016/j.eps1. 2016.01.027

Pfeffer, J., Spada, G., Mémin, A., Boy, J., \& Allemand, P. (2017). Decoding the origins of vertical land motions observed today at coasts. Geophysical Journal International, 210(1), 148165. https://doi.org/10.1093/gji/ggx142

Ponte Rui M., Carson Mark, Cirano Mauro, Domingues Catia M., et al. (2019). Towards Comprehensive Observing and Modeling Systems for Monitoring and Predicting Regional to Coastal Sea Level. Frontiers in Marine Science. https://doi.org/10.3389/fmars.2019.00437

Raucoules, D., G. Le Cozannet, G. Wöppelmann, M. de Michele, M. Gravelle, A. Daag, \& M. Marcos (2013). High nonlinear urban ground motion in Manila (Philippines) from 1993 to 2010 observed by DIn-SAR: Implications for sea-level measurement, Remote Sens. Environ., 139, 386-397. https://doi.org/10.1016/j.rse.2013.08.021

Rezvani, M.-H., Sabbagh A., \& Ardalan A.A. (2015). Robust automatic reduction of multibeam bathymetric data based on M-estimators. Marine Geodesy 38(4), 327-344. https://doi.org/10. $\underline{1080 / 01490419.2015 .1053639}$

Rezvani, M.-H., Watson, C.S., King, M.A. (2021). Estimating Vertical Land Motion and Residual Altimeter Systematic Errors Using a Kalman-Based Approach. Journal of Geophysical Research: Oceans. 126(6), e2020JC017106. https://doi.org/10.1029/2020JC017 $\underline{106}$

Riddell, A. R., King, M. A., \& Watson, C. S. (2020). Present-day vertical land motion of Australia from GPS observations and geophysical models. Journal of Geophysical Research: Solid Earth,125, e2019JB018034. https://doi.org/10.1029/2019JB018034 
Riddell, A.R., King, M.A., \& Watson, C.S. (2021). Ongoing postseismic vertical deformation of the Australian continent from far-field earthquakes. Geophysical Journal International, In review. https://doi.org/10.1002/essoar.10504158.1

Ridgway, K., \& Hill, K. (2009) The East Australian Current. In A Marine Climate Change Impacts and Adaptation Report Card for Australia 2009 (Eds. E.S. Poloczanska, A.J. Hobday and A.J. Richardson), NCCARF Publication 05/09, ISBN 978-1-921609-03-9.

Santamaría-Gómez, A., \& Mémin, A. (2015). Geodetic secular velocity errors due to interannual surface loading deformation, Geophysical Journal International, 202(2), 763-767, https://doi. org/10.1093/gji/ggv190

Santamaría-Gómez, A., Gravelle, M., Collilieux, X., Guichard, M., Míguez M. B., Tiphaneau P., \& Wöppelmann, G. (2012). Mitigating the effects of vertical land motion in tide gauge records using state-of-the-art GPS velocity field, Global and Planetary Change, 98-99, 6-17. https://doi.org/10.1016/j.gloplacha.2012.07.007

Santamaría-Gómez, A., Gravelle, M.; Dangendorf, S.; Marcos, M.; Spada, G.; \& Wöppelmann, G. (2017). Uncertainty of the 20th century sea-level rise due to vertical land motion errors. Earth and Planetary Science Letters, 473: 24-32. https://doi.org/10.1016/i.eps1.2017.05.038

Scharroo, R., Leuliette, E. W., Lillibridge, J. L., Byrne, D., Naeije, M. C., \& Mitchum, G. T. (2013). RADS: Consistent multi-mission products, in Proceedings Symposium on 20 Years of Progress in Radar Altimetry, European Space Agency, Special Publication SP-710.

Sun, Y., \& Riva, R. E. M. (2020). A global semi-empirical glacial isostatic adjustment (GIA) model based on Gravity Recovery and Climate Experiment (GRACE) data, Earth System Dynamics, 11, 129-137. https://doi.org/10.5194/esd-11-129-2020

Suthers I.M., Young J.W., Baird M.E., Roughan M., Everett J.D., Brassington G.B., Byrne M., Condie S.A., Hartog J.R., Hassler C.S., Hobday A.J., Holbrook N.J. (2011) The strengthening East Australian Current, its eddies and biological effects - an introduction and overview. DeepSea Research Part II: Topical Studies in Oceanography, 58(5):538-546. https://doi.org/10.1016/j.dsr2.2010.09.029

Theobald, A., \& McGowan, H. (2016). Evidence of increased tropical moisture in southeast Australian alpine precipitation during ENSO, Geophysical Research Letters, 43, 10, 901-10, 908. https://doi.org/10.1002/2016GL070767

Tregoning, P., Watson, C.S., Ramillien G., McQueen H., \& Zhang J. (2009). Detecting hydrologic deformation using GRACE and GPS, Geophysical Research Letters, 36, L15401. https://doi.org/10.1029/2009GL038718

Twidale CR. (2011). Is Australia a tectonically stable continent? Analysis of a myth and suggested morphological evidence of tectonism. Progress in Physical Geography: Earth and Environment. 35(4), 493-515. https://doi.org/10.1177/0309133311402715

Wang, J., Church, J.A., Zhang, X., Chen, X. (2021). Reconciling global mean and regional sea level change in projections and observations. Nature Communications, 12(1), 990. https://doi.org/10.1038/s41467-021-21265-6

Watson, C. S., Legresy, B., Beardsley, J., Zhou, A., \& King, M. A. (2020). Altimeter validation results from the Bass Strait validation facility, Australia. Ocean Surface Topography Science 
Team Meeting 2020, Virtual Forum. Retrieved from https://ostst.aviso.altimetry.fr/fileadmin/ user_upload/tx ausyclsseminar/files/OSTST2020_Absolute Bias_Watson_et_al.pdf

Watson, C.S., Burgette, R., Tregoning, P., White, N., Hunter, J., Coleman, R., Handsworth, R., \& Brolsma, H. (2010). Twentieth century constraints on sea level change and earthquake deformation at Macquarie Island. Geophysical Journal International, 182(2), 781-796. https://doi.org/10.1111/j.1365-246X.2010.04640.x

Watson, C.S., White, N., Church, J., Burgette, R., Tregoning, P., \& Coleman, R., (2011). Absolute calibration in Bass Strait, Australia: TOPEX, Jason-1 and OSTM/Jason-2. Marine Geodesy, 34 (3-4), 242-260. https://doi.org/10.1080/01490419.2011.584834

Watson, C.S., White, N.J., Church, J.A., King, M.A., Burgette, R.J., \& Legresy, B. (2015). Unabated global mean sea-level rise over the satellite altimeter era. Nature Climate Change, 5(6), 565-568. https://doi.org/10.1038/nclimate2635

Watson, P.J. (2020). Updated Mean Sea-Level Analysis: Australia. Journal of Coastal Research, 36(5), 915-931. https://doi.org/10.2112/JCOASTRES-D-20-00026.1

White, N.J., Haigh, I.D., Church, J.A., Koen, T., Watson, C.S., Pritchard, T.R., Watson, P.J., Burgette, R.J., McInnes, K.L., You, Z.-J., Zhang, X., \& Tregoning, P. (2014). Australian sea levels-Trends, regional variability and influencing factors. Earth-Science Reviews. 136, 155174. https://doi.org/10.1016/j.earscirev.2014.05.011

Williams, S.D.P., Bock, Y., Fang, P., Jamason, P., Nikolaidis, R.M., Prawirodirdjo, L., Miller, M., \& Johnson, D.J. (2004). Error analysis of continuous GPS position time series. Journal of Geophysical Research: Solid Earth, 109(3), 1-19. https://doi.org/10.1029/2003JB002741

Wöppelmann, G., Pouvreau, N., Coulomb, A., Simon, B., \& Woodworth, P. L. (2008). Tide gauge datum continuity at Brest since 1711: France's longest sea-level record. Geophysical Research Letters, 35, L22605. https://doi.org/10.1029/2008GL035783

Wu, X., Ray, J., \& T. van Dam (2012), Geocenter motion and its geodetic and geophysical implications, Journal of Geodynamics, 58, 44-61. https://doi.org/10.1016/j.jog.2012.01.007

Wöppelmann, G., \& Marcos, M. (2016). Vertical land motion as a key to understand sea level change and variability. Reviews of Geophysics, 54(1), 64-92. https://oi.org/10.1002/ $\underline{2015 \operatorname{rg} 000502}$

Wöppelmann, G., Letetrel, C., Santamaría-Gómez, A., Bouin, M.-N., Collilieux, X., Altamimi, Z., Williams, S. D. P. \& Martin Miguez, B. (2009). Rates of sea-level change over the past century in a geocentric reference frame, Geophysical Research Letters, 36, L12607. https://doi.org/10.1029/2009GL038720

Yin, W. , Li, T. , Zheng, W., Hu, L. , Han, S.-C. , Tangdamrongsub, N. , Šprlák, M., \& Huang, Z. (2020). Improving regional groundwater storage estimates from GRACE and global hydrological models over Tasmania, Australia. Hydrogeology Journal, 28, 1809-1825. https://doi.org/10.1007/s10040-020-02157-3 
Table S1. The remaining geophysical and environmental corrections applied to the mission-specific ASL

\begin{tabular}{|l|l|l|l|l|l|}
\hline \multirow{2}{*}{ Mission } & Orbit & Sea state bias & $\begin{array}{l}\text { Dry } \\
\text { troposphere }\end{array}$ & $\begin{array}{l}\text { Wet } \\
\text { troposphere }\end{array}$ & Ionosphere \\
\cline { 2 - 6 } TOPEX & GSFC-Std1204 & $\begin{array}{l}\text { Chambers } \\
\text { BM4-parametric }\end{array}$ & ECMWF & Radiometer & $\begin{array}{l}\text { Smoothed } \\
\text { dual- } \\
\text { frequency }\end{array}$ \\
\hline Jason-1 & CNES-GDRE & CLS-nonparametric & ECMWF & Radiometer & $\begin{array}{l}\text { Smoothed } \\
\text { dual- } \\
\text { frequency }\end{array}$ \\
\hline $\begin{array}{l}\text { OSTM/ } \\
\text { Jason-2 }\end{array}$ & CNES-GDRE & $\begin{array}{l}\text { CLS-nonparametric- } \\
\text { MLE3 }\end{array}$ & ECMWF & Radiometer & $\begin{array}{l}\text { Smoothed } \\
\text { dual- } \\
\text { frequency- } \\
\text { MLE3 }\end{array}$ \\
\hline Jason-3 & CNES-GDRE & $\begin{array}{l}\text { CLS-nonparametric- } \\
\text { MLE3 }\end{array}$ & ECMWF & Radiometer & $\begin{array}{l}\text { Smoothed } \\
\text { dual- } \\
\text { frequency- } \\
\text { MLE3 }\end{array}$ \\
\hline ERS-2 & $\begin{array}{l}\text { GFZ-SLCCI- } \\
\text { VER11 }\end{array}$ & $\begin{array}{l}\text { Gaspar } \\
\text { BM3-parametric }\end{array}$ & ECMWF & Radiometer & NIC09 \\
\hline Envisat & CNES-GDRD & CLS-nonparametric & ECMWF & Radiometer & JPL-GIM \\
\hline $\begin{array}{l}\text { SARAL/ } \\
\text { AltiKa }\end{array}$ & CNES-GDRE & NOAA-hybrid & ECMWF & Radiometer & JPL-GIM \\
\hline Sentinel-3A & CNES-GDRF & $\begin{array}{l}\text { Tran2012- } \\
\text { nonparametric }\end{array}$ & ECMWF & Radiometer & $\begin{array}{l}\text { Smoothed } \\
\text { dual- } \\
\text { frequency }\end{array}$ \\
\hline
\end{tabular}

Table S2. RMSE and distance thresholds used to reduce the number of AXO observations for computational efficiency.

\begin{tabular}{|c|c|c|c|c|}
\hline \multirow[b]{2}{*}{ Crossover } & \multirow[b]{2}{*}{ Overflying missions } & \multicolumn{2}{|c|}{ Threshold } & \multirow[b]{2}{*}{ \# of observations } \\
\hline & & $\begin{array}{l}\text { RMSE } \\
(\mathbf{m m})\end{array}$ & $\begin{array}{l}\text { Distance } \\
(\mathbf{k m})\end{array}$ & \\
\hline \multirow{4}{*}{ Tandem } & TOPEX-side B \& Jason-1 & 19 & 150 & 102 \\
\hline & Jason-1 \& Jason-2 & 19 & 150 & 102 \\
\hline & Jason-2 \& Jason-3 & 19 & 150 & 102 \\
\hline & ERS-2 \& Envisat & 35 & 200 & 105 \\
\hline \multirow{7}{*}{ Dual } & ERS-2 \& TOPEX-side A & 84 & 350 & 134 \\
\hline & ERS-2 \& TOPEX-side B & 84 & 350 & 134 \\
\hline & ERS-2 \& Jason-1 & 84 & 350 & 134 \\
\hline & Envisat \& Jason-1 & 84 & 350 & 134 \\
\hline & Envisat \& Jason-2 & 84 & 350 & 134 \\
\hline & SARAL \& Jason-2 & 84 & 350 & 134 \\
\hline & Sentinel-3A \& Jason-3 & 60 & 350 & 121 \\
\hline
\end{tabular}


manuscript submitted to Journal of Geodesy

1407

1408

1409

Table S3. Rule set proposed to adapt process noise of time-variable intercepts, time-fixed VLMs, and timevariable VLMs according to trends GPS residuals.

\begin{tabular}{|c|c|c|c|c|}
\hline $\begin{array}{c}\text { Residual trend } \\
\text { bound } \\
(\mathbf{m m} / \mathbf{y r})\end{array}$ & $\begin{array}{c}\text { Intercept } \\
\text { process }\end{array}$ & $\begin{array}{c}\text { Linear VLM } \\
\text { process noise } \\
(\mathbf{m m} / \mathbf{y r} \sqrt{\mathbf{s}})\end{array}$ & $\begin{array}{c}\text { Non-linear VLM } \\
\text { process noise } \\
(\mathbf{m m} / \sqrt{\mathbf{s}})\end{array}$ \\
\cline { 1 - 3 } Lower & Upper & noise $(\mathbf{m m} / \sqrt{\mathbf{s})}$ & $0.001000 / \sqrt{9.9}$ & $0.871875 / \sqrt{9.9}$ \\
\hline 0 & 1 & $1.089844 / \sqrt{9.9}$ & $0.003037 / \sqrt{9.9}$ & $0.939648 / \sqrt{9.9}$ \\
\hline 1 & 2 & $1.174560 / \sqrt{9.9}$ & $0.007406 / \sqrt{9.9}$ & $1.007422 / \sqrt{9.9}$ \\
\hline 2 & 3 & $1.259277 / \sqrt{9.9}$ & $0.010854 / \sqrt{9.9}$ & $1.068750 / \sqrt{9.9}$ \\
\hline 3 & 4 & $1.335937 / \sqrt{9.9}$ & $0.013282 / \sqrt{9.9}$ & $1.139062 / \sqrt{9.9}$ \\
\hline 4 & 5 & $1.423828 / \sqrt{9.9}$ & $0.016926 / \sqrt{9.9}$ & $1.236328 / \sqrt{9.9}$ \\
\hline 5 & 6 & $1.545410 / \sqrt{9.9}$ & $0.020785 / \sqrt{9.9}$ & $1.326172 / \sqrt{9.9}$ \\
\hline 6 & 7 & $1.657715 / \sqrt{9.9}$ & $0.024539 / \sqrt{9.9}$ & $1.393945 / \sqrt{9.9}$ \\
\hline 7 & 8 & $1.742432 / \sqrt{9.9}$ & $0.027759 / \sqrt{9.9}$ & $1.458398 / \sqrt{9.9}$ \\
\hline 8 & 9 & $1.822998 / \sqrt{9.9}$ & $0.030979 / \sqrt{9.9}$ & $1.522852 / \sqrt{9.9}$ \\
\hline 9 & 10 & $1.903564 / \sqrt{9.9}$ & & \\
\hline
\end{tabular}

1410

1411 


\begin{tabular}{|c|} 
Observational series \\
$>$ ALT minus TG (ATG) series. \\
$>$ ALT crossover (AXO) series. \\
$>$ GPS bedrock height series. \\
$>$ Pseudo-observation constraints.
\end{tabular}

Geostatistical information

> Covariance of ASL residuals from semi-variogram analysis.

> Covariance of RSL residuals from semi-variogram analysis.

D Covariance of height residuals from semi-variogram analysis.

$>$ Covariance of unknowns from semi-variogram analysis (optional).

> Noise content of observational residuals from spectral analysis.

> Transition coefficients for time-correlated noise from spectral analysis.

\section{Solution 1}

Aim: Derive a priori estimates of linear bias drift

> Use tight constraints on a priori estimates of GPS VLM trend from Hector.

> Use tight constraints on a priori estimates of TG VLM trends from GPS-Krig.

$>$ Use tight constraints on zero-value a priori estimates of non-linear VLM.

$>$ Use large uncertainty ( $\pm 5 \mathrm{~mm} / \mathrm{yr}$ ) for initial values of linear bias drift.

$>$ Use tight process noise $\left(10^{-3} / \sqrt{9.9} \mathrm{~mm} / \mathrm{yr} \sqrt{\mathrm{s}}\right)$ for linear VLMs and bias drifts.

$>$ Use tight process noise $\left(10^{-6} / \sqrt{9.9} \mathrm{~mm} / \mathrm{km} \sqrt{\mathrm{s}}\right)$ for ASL slopes from mean sea surface.

$>$ Use loose process noise $(1 / \sqrt{9.9} \mathrm{~mm} / \sqrt{\mathrm{s}})$ for time-variable intercepts.

$>$ Use AR1-derived process noise and initial uncertainty $( \pm 10 \mathrm{~mm})$ for time-correlated noise.

$>$ Iterate with output as new a priori state and adaptive process noise of time-variable intercepts from trends in ATG and GPS residuals (Table S3).

\section{Solution 2}

Aim: Improve a priori estimates of linear VLM

$>$ Use tight constraints on linear bias drift estimates from Solution 1.

$>$ Use tight process noise $\left(10^{-3} / \sqrt{9.9} \mathrm{~mm} / \mathrm{yr} \sqrt{\mathrm{s}}\right)$ for linear bias drifts.

$>$ Use tight constraints on zero-value a priori estimates of non-linear VLM.

$>$ Use loose process noise $\left(10^{-2} / \sqrt{9.9} \mathrm{~mm} / \mathrm{yr} \sqrt{\mathrm{s}}\right)$ for linear GPS VLMs from Hector.

$>$ Use loose process noise $\left(10^{-2} / \sqrt{9.9} \mathrm{~mm} / \mathrm{yr} \sqrt{\mathrm{s}}\right)$ for linear TG VLMs from GPS-Krig.

$>$ Use tight process noise $(10-6 / \sqrt{9.9} \mathrm{~mm} / \mathrm{km} \sqrt{\mathrm{s}})$ for ASL slopes.

$>$ Use tight process noise $\left(10^{-6} / \sqrt{9.9} \mathrm{~mm} / \sqrt{\mathrm{s}}\right)$ for time-invariable intercepts from Solution 1 .

> Use AR1-derived process noise and initial uncertainty $( \pm 10 \mathrm{~mm})$ for time-correlated noise.

$>$ Iterate with output as new a priori state and adaptive process noise of VLM from trends in ATG and GPS residuals (Table S3).

\section{Solution 3}

Aim: Optimize a posteriori estimates of non-linear evolution in VLM and bias drift

Use tight constraints on estimates of TG and GPS VLM trend from Solution 2.

$>$ Use loose constraints on zero-value a priori non-linear VLMs.

$>$ Use large uncertainty ( $\pm 5 \mathrm{~mm} / \mathrm{yr}$ ) for initial values of non-linear bias drifts.

$>$ Use large uncertainty $( \pm 10 \mathrm{~mm})$ for non-linear VLMs.

$>$ Use tight process noise $\left(10^{-3} / \sqrt{9.9} \mathrm{~mm} / \mathrm{yr} \sqrt{\mathrm{s}}\right)$ for linear VLMs from Solution 2 .

$>$ Use loose process noise $\left(2.88 \times 10^{-1} / \sqrt{9.9} \mathrm{~mm} / \mathrm{yr} \sqrt{\mathrm{s}}\right)$ for non-linear bias drifts.

$>$ Use loose process noise $(1 / \sqrt{9.9} \mathrm{~mm} / \sqrt{\mathrm{s}})$ for non-linear VLMs.

$>$ Use a priori states and uncertainties from Solution 2.

$>$ Use tight process noise $\left(10^{-6} / \sqrt{9.9} \mathrm{~mm} / \mathrm{km} \sqrt{\mathrm{s}}\right)$ for ASL slopes.

$>$ Use tight process noise $\left(10^{-6} / \sqrt{9.9} \mathrm{~mm} / \sqrt{\mathrm{s}}\right)$ for time-invariable intercepts from Solution 1 .

$>$ Use AR1-derived process noise and initial uncertainty $( \pm 10 \mathrm{~mm})$ for temporal-correlated noise.

$>$ Iterate with output as new a priori state, loose constraints on new bias drifts, and adaptive process noise of non-linear VLM from trends in GPS residuals (Table S3).

Figure S1. A flow illustration of multi-stage implementation strategy to estimates unknowns in an iterative manner. The solutions commenced with estimating a priori estimates of linear bias drifts from a priori knowledge about linear VLMs at TG and GPS sites, then rectified a priori linear VLMs, and concluded 

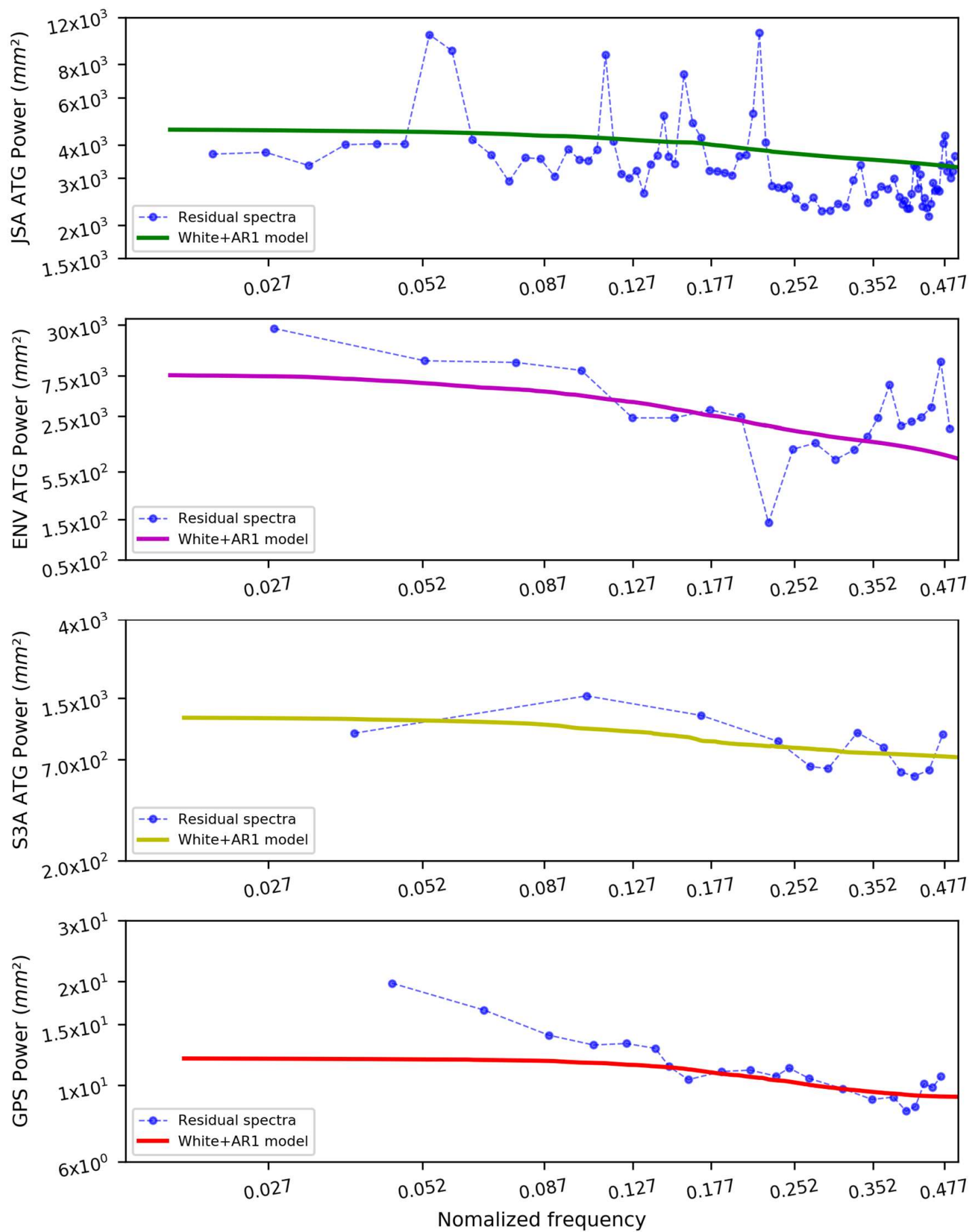

Figure S2. Median power spectral density of input dataset for (top) ATG in the case of (top row) Jasonseries, (second row) Envisat-series, and (third row) Sentinel-3A, and for (bottom row) GPS height observations. Note the "white plus AR1" noise model is a quite reasonable fit to the ATG observations, yet the peaks indicate the presence of residual tides. This model slightly underpredicts the low-frequency energy in the GPS observations at the low-frequency end. 

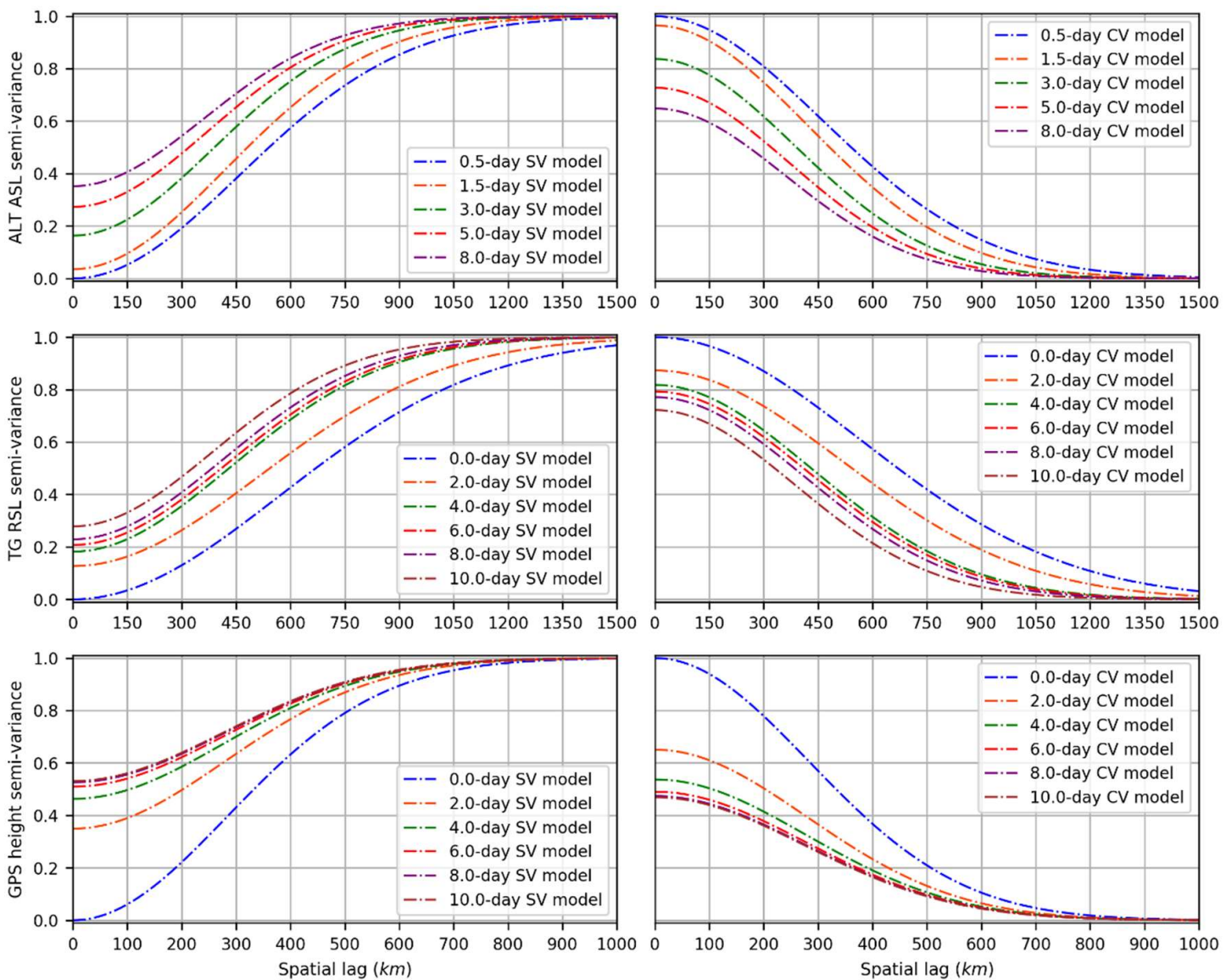

Figure S3. Gaussian negative-definite semi-variograms (SV, left panel) and positive-definite covariograms (CV, right panel) with models derived from semi-variances of (top) ALT ASL, (middle) TG RSL and (bottom) GPS height residuals around the Australian region. Note the nugget effects are relative to the semi-variance estimates at zero-lag in space and time. 

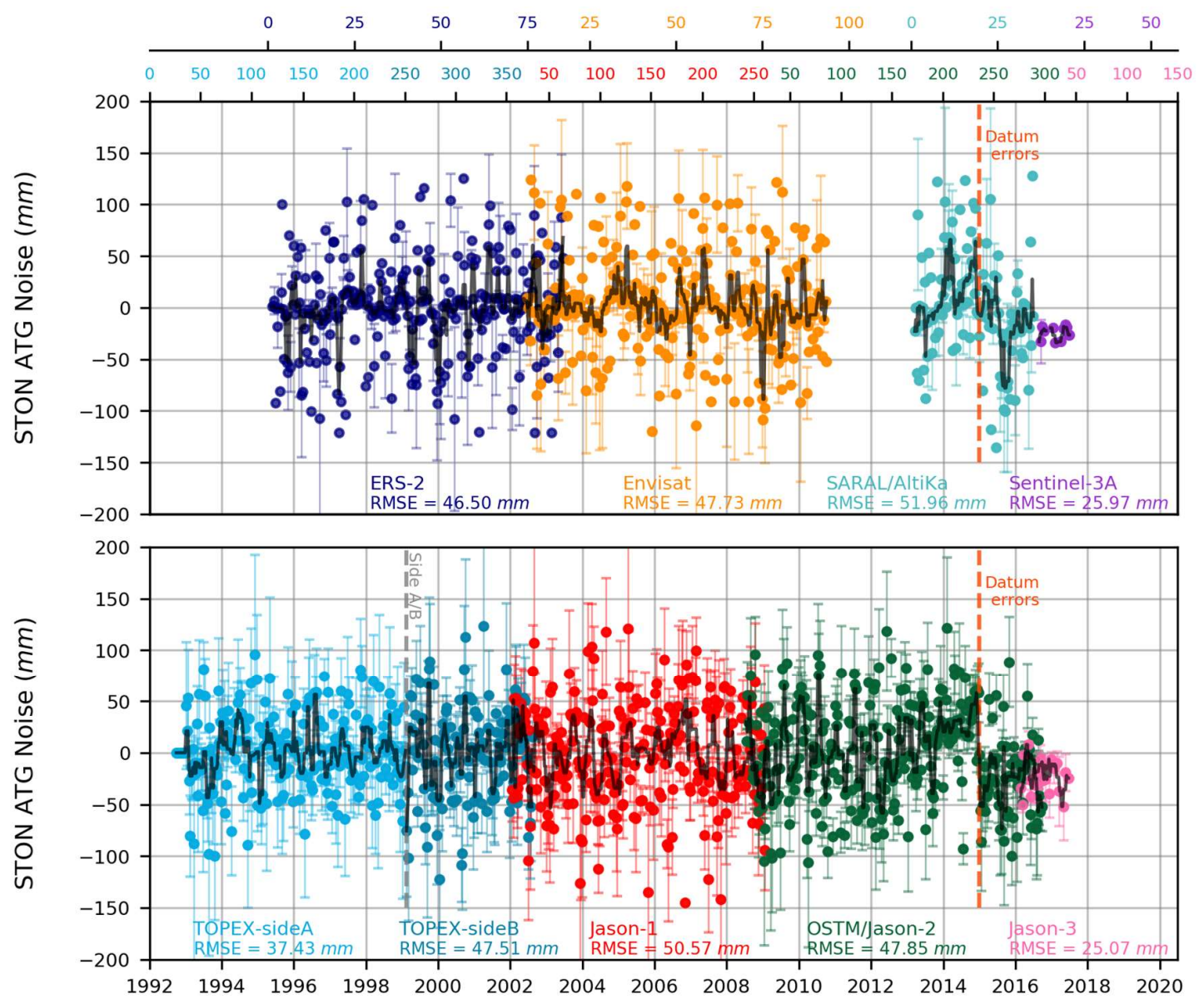

Figure S4. Uncovered datum errors of $\sim \pm 25 \mathrm{~mm}$ in RSL data recorded at STON TG commenced since $\sim 2015$, that were detected using cycle-by-cycle weighted average of the ATG "white plus AR1" residuals. The dashed orange lines specify the temporal margins of these datum shifts. 


\section{Supplementary Results and discussion}

\subsection{Linear VLM}

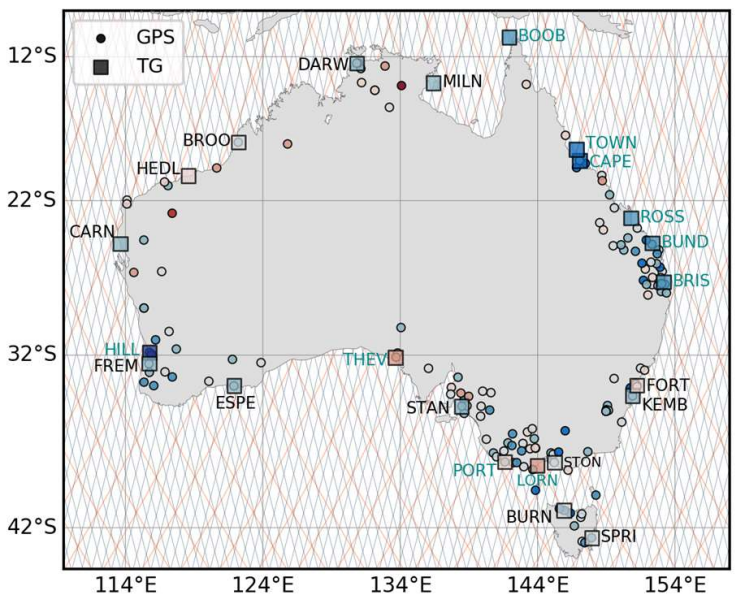

(a) Our VLM estimate

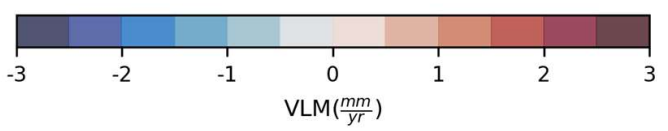

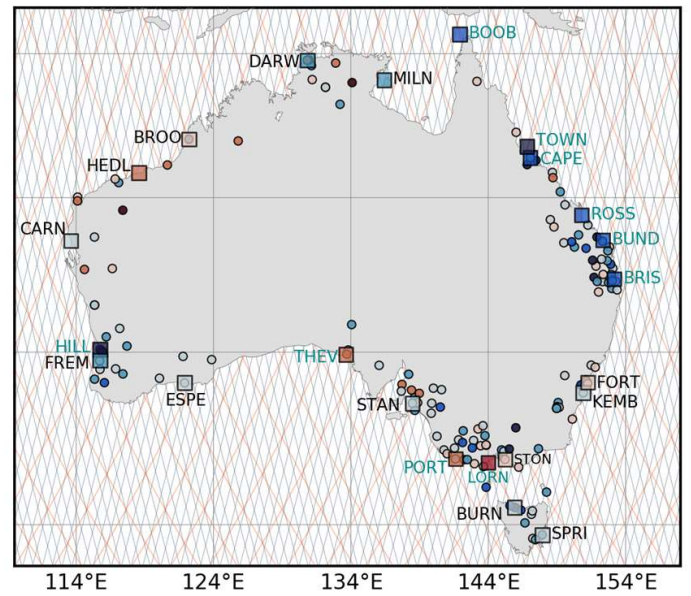

(b) VLM difference

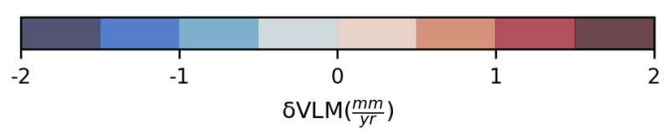

Figure S5. Map of (a) our estimates of linear VLMs, and (b) differences of our approach minus ICE6G_D GIA at tide gauge (TG, squares) and GPS sites (circles). TGs with significant differences at 1-sigma are annotated in cyan. For clarity, TG latitude at TOWN and FREM locations are shifted by +0.75 and -0.45 degrees, respectively. The ground tracks of Jason-series and Envisat-series altimeters are shown in orange and cyan, respectively.

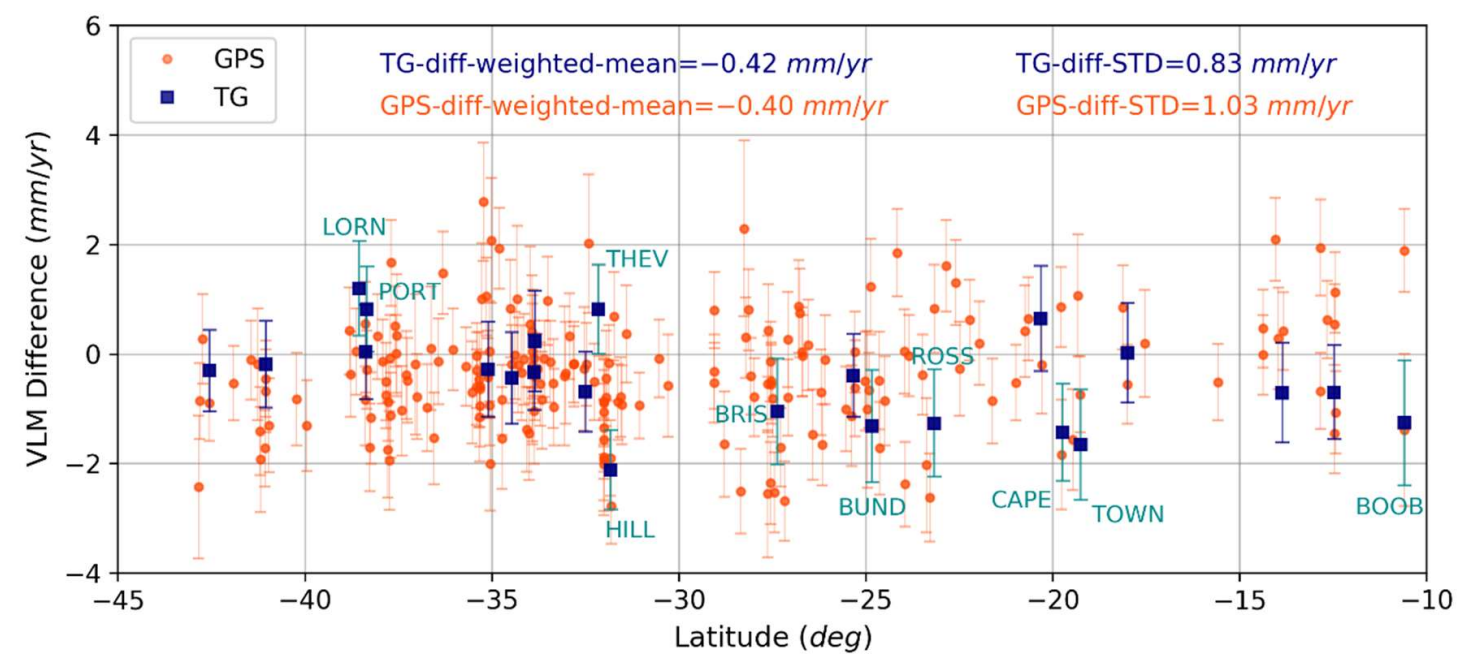

Figure S6. Profile of VLM differences per latitude, our estimates minus ICE6G_D at TGs (blue squares) and GPS sites (orange circles). TGs with significant differences at 1-sigma are annotated in cyan. For clarity, TG latitudes at CAPE, CARN and FREM locations are shifted by -0.45 degrees, respectively. 


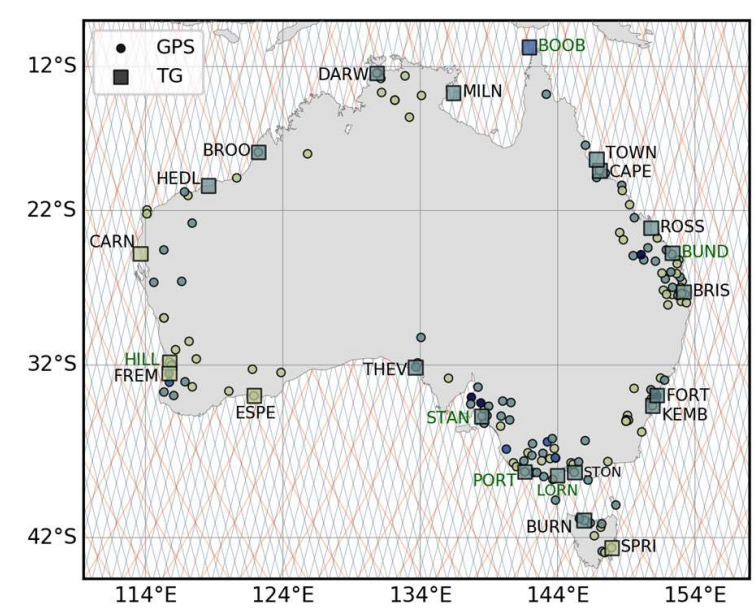

(a) Our VLM uncertainty

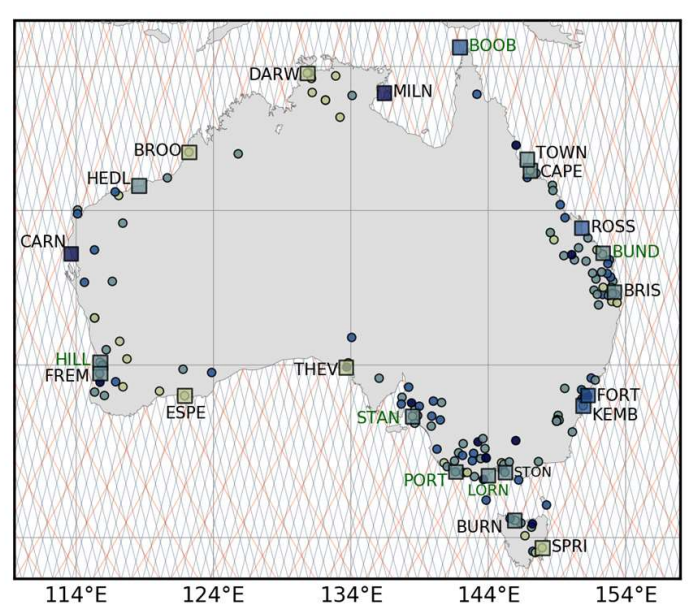

(b) GPS-Krig \& Hector VLM uncertainty

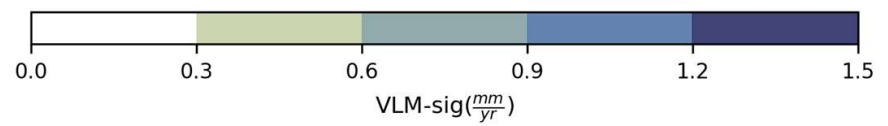

Figure S7. Map of (a) our VLM uncertainty estimates, scaled by a posteriori variance factor, against (b) those from Hector at GPS and GPS-Krig at TG sites. The ground tracks of Jason-series and Envisat-series altimeters are shown in orange and cyan, respectively.

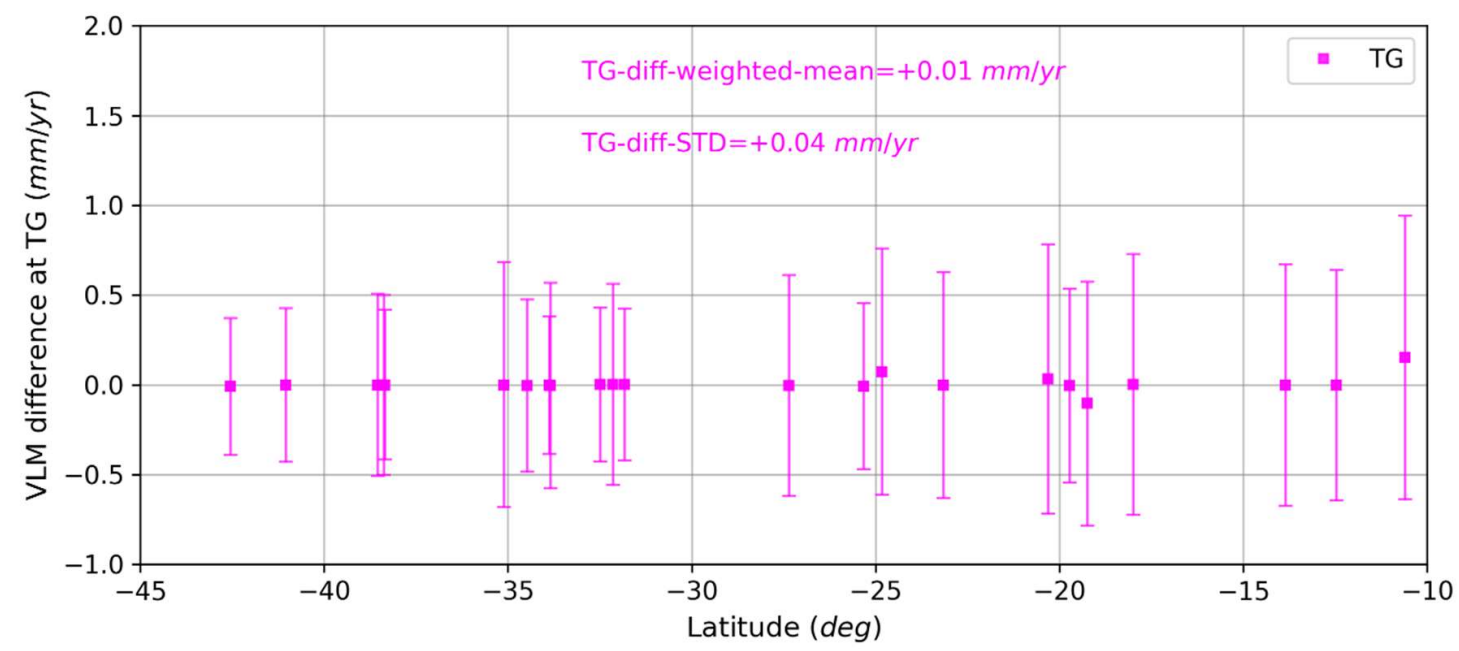

Figure S8. Profile of multi-mission VLM differences at TGs, our revisited outputs (constraining on the averages of time-variable bias drifts derived from Solution 3) minus our preferred estimates (constraining on the averages of time-fixed bias drifts from Solution 1), as a function of latitude. Note negligible differences which suggests our method is appropriate. Error bars are \pm 1 -sigma scaled by the a posteriori variance factor. For clarity, TG latitudes at CAPE, CARN and FREM locations are shifted by -0.45 degrees. 


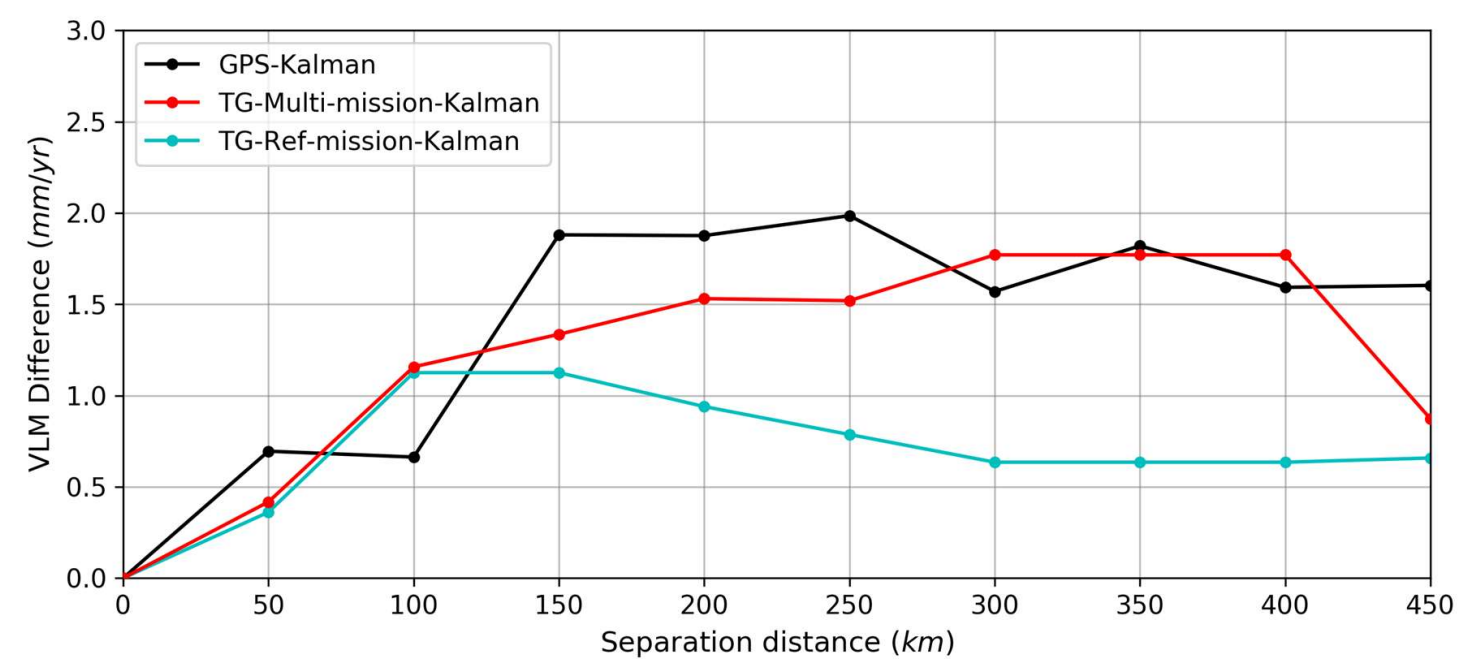

Figure S9. Spatial variability in VLM as a function of separation distance between TG sites from multimission (red) and reference-mission (cyan) solutions, compared to those from GPS sites (black) as the benchmark. Note over reasonably short scales, the multi-mission solution tends to show closer to GPS in terms of variability compared to the reference-mission-only implementation. For comparison purposes, the nugget effects are removed.

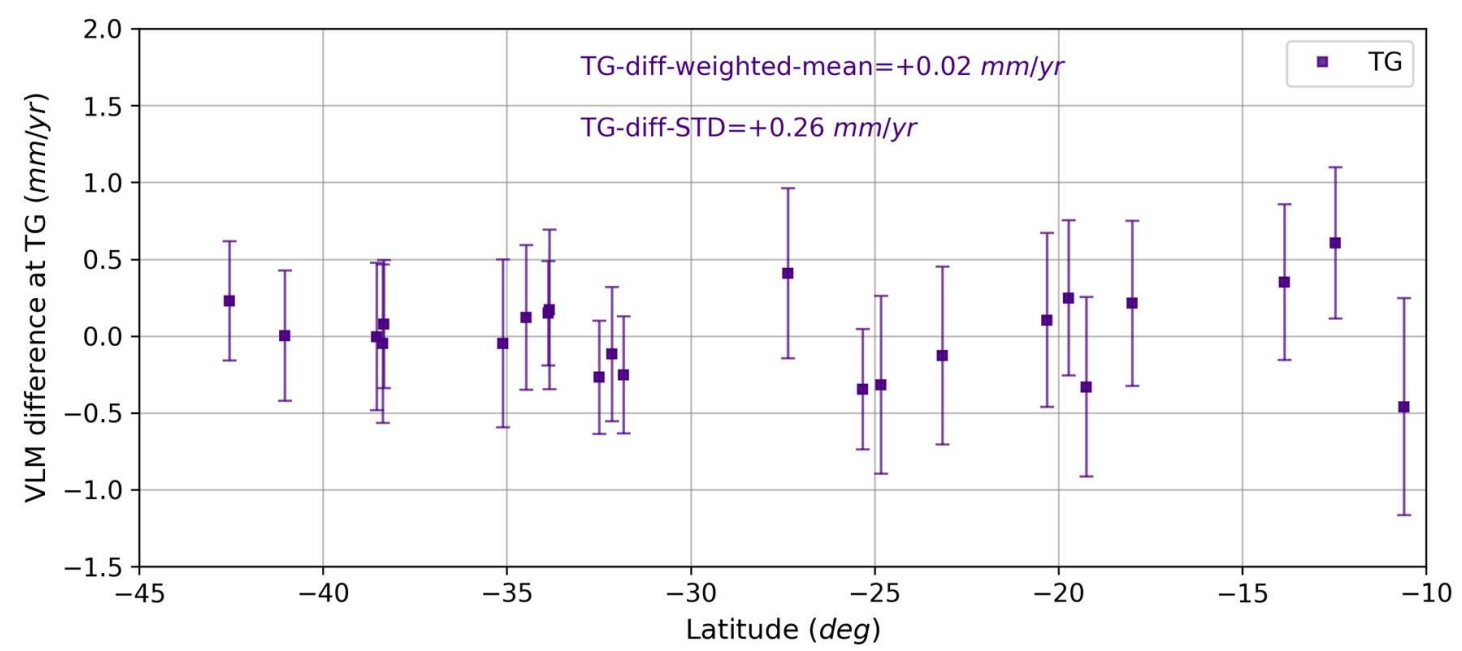

1472 Figure S10. Profile of VLM differences from the multi-mission and reference-mission solutions, as a function of latitude. Note significant differences in TG VLM at higher latitudes. Error bars are \pm 1 -sigma scaled by the a posteriori variance factor. For clarity, TG latitudes at CAPE, CARN and FREM locations are shifted by -0.45 degrees. 

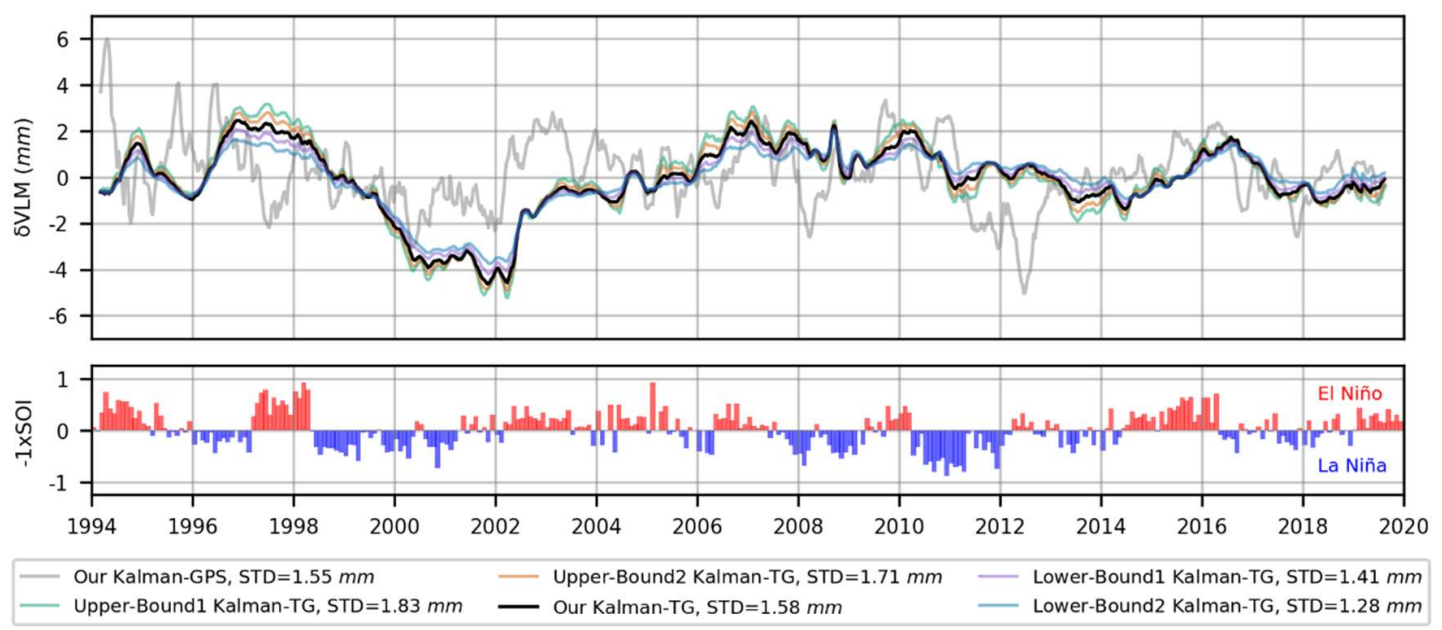

Figure S11. Stack of non-linear variabilities in TG VLMs (in black) from our preferred solution against upper and lower bounded solutions, along with stacked non-linear VLMs at GPS sites (in gray). Note the process noise for non-linear VLM at TGs tuned, such that the dispersion of the averaged stacked non-linear VLM from TGs closely matches that from GPS sites. For comparison, Southern Oscillation Index (SOI)

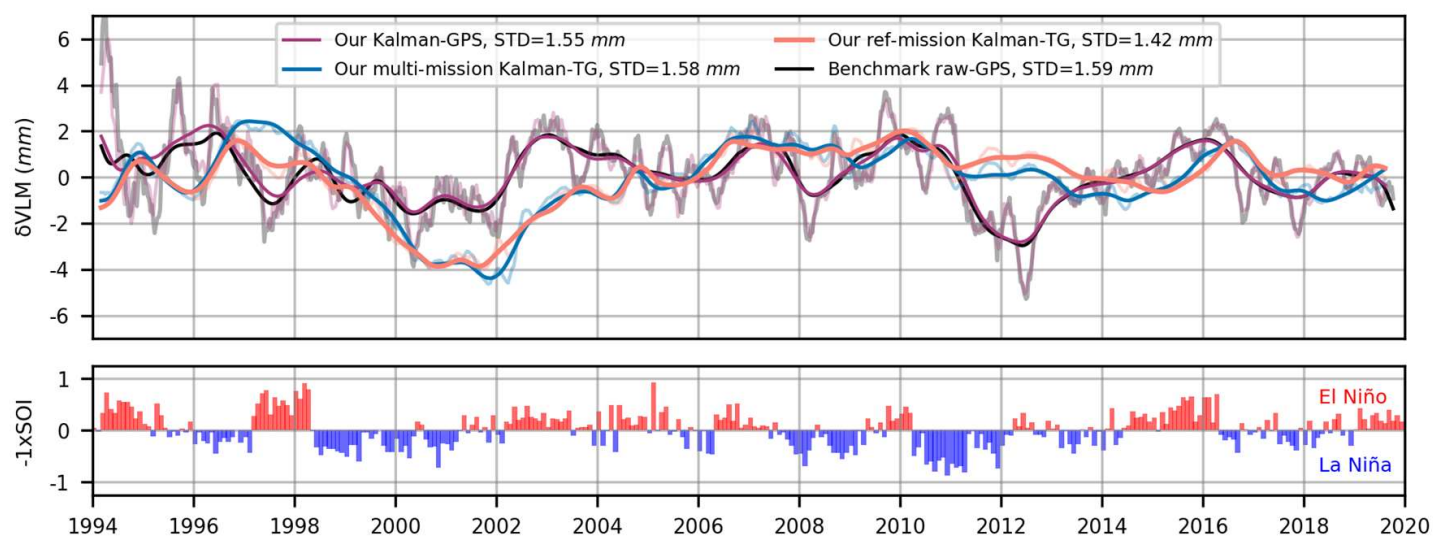

Figure S12. Weighted average stack of our estimates of non-linear VLMs at TGs from reference-mission (pink line) and multi-mission (blue line) solutions, and coastal GPS sites (purple line) over the Australian continent, with respect to the control stack derived from detrended GPS height series (black line). For comparison, Southern Oscillation Index (SOI) with the sign reversed is shown in the lower panel as the climatic descriptor in the region. 

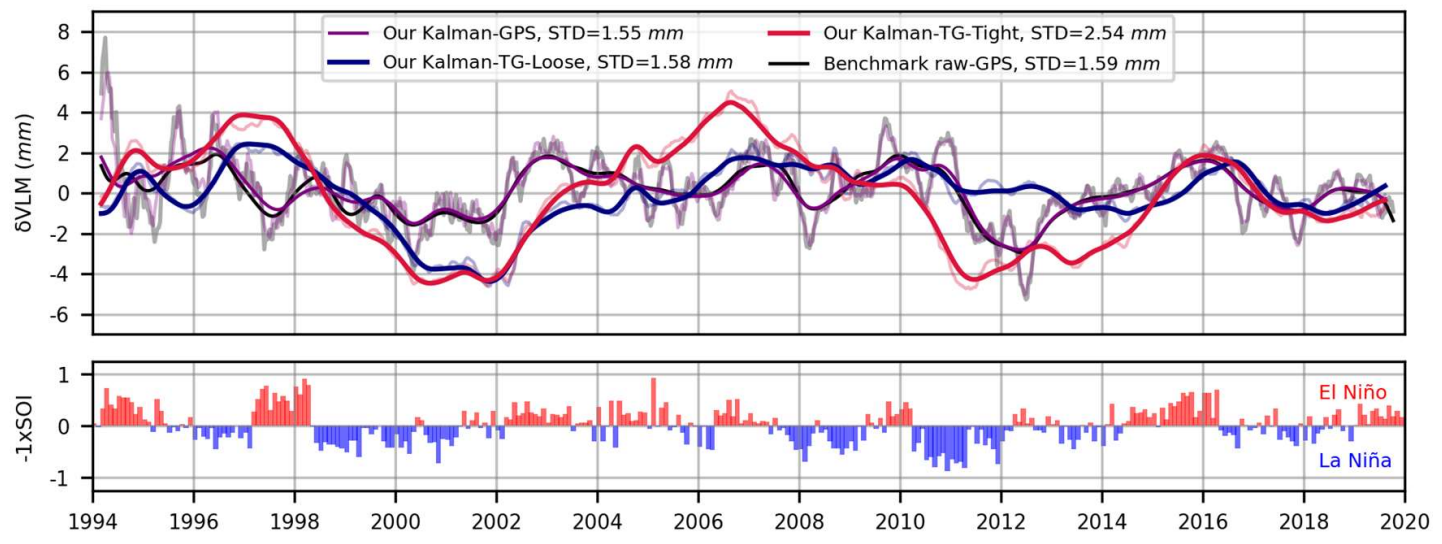

Figure S13. Weighted average stack of our estimates of evolving VLMs at TGs with linear (red line) and non-linear (blue line) estimates of bias drift, and coastal GPS sites (purple line) over the Australian continent, with respect to the control stack derived from detrended GPS height series (black line). For comparison, Southern Oscillation Index (SOI) with the sign reversed is shown in the lower panel as the climatic descriptor in the region.
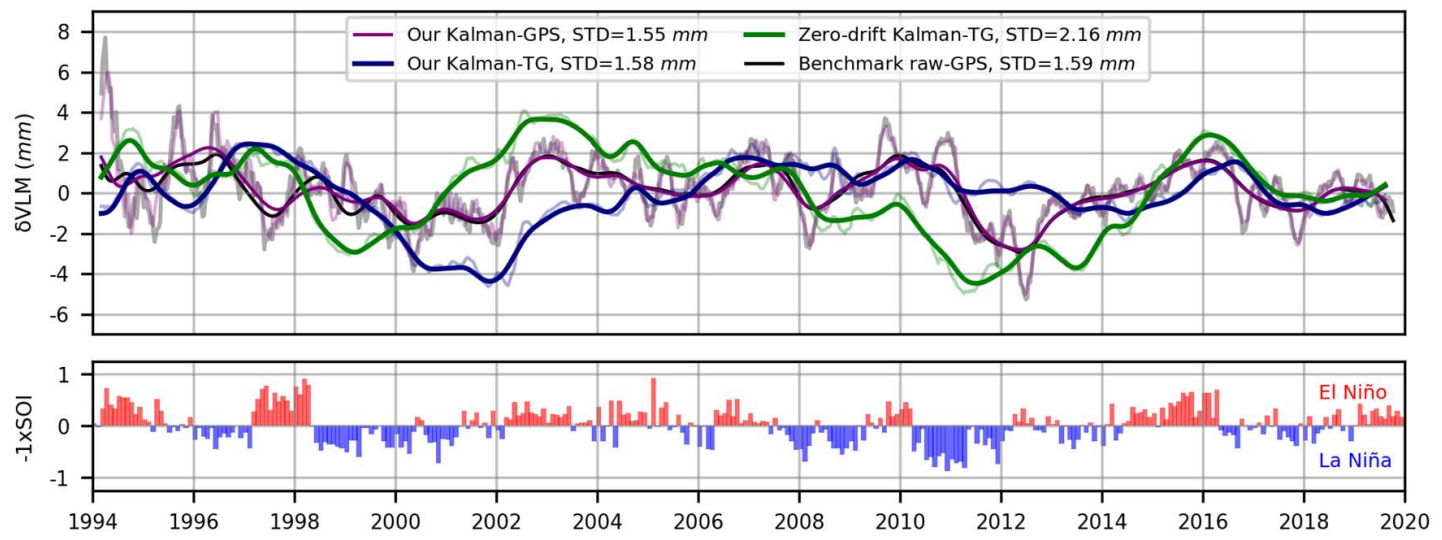

Figure S14. Weighted average stack of our estimates of non-linear VLMs at TGs from our preferred multimission solution (blue line), and the solution when a zero-drift assumption imposed across altimetry span (green line), and coastal GPS sites (purple line) over the Australian continent, with respect to the control stack derived from detrended GPS height series (black line). For comparison, Southern Oscillation Index (SOI) with the sign reversed is shown in the lower panel as the climatic descriptor in the region. 


\subsection{Non-linear altimeter systematic errors}
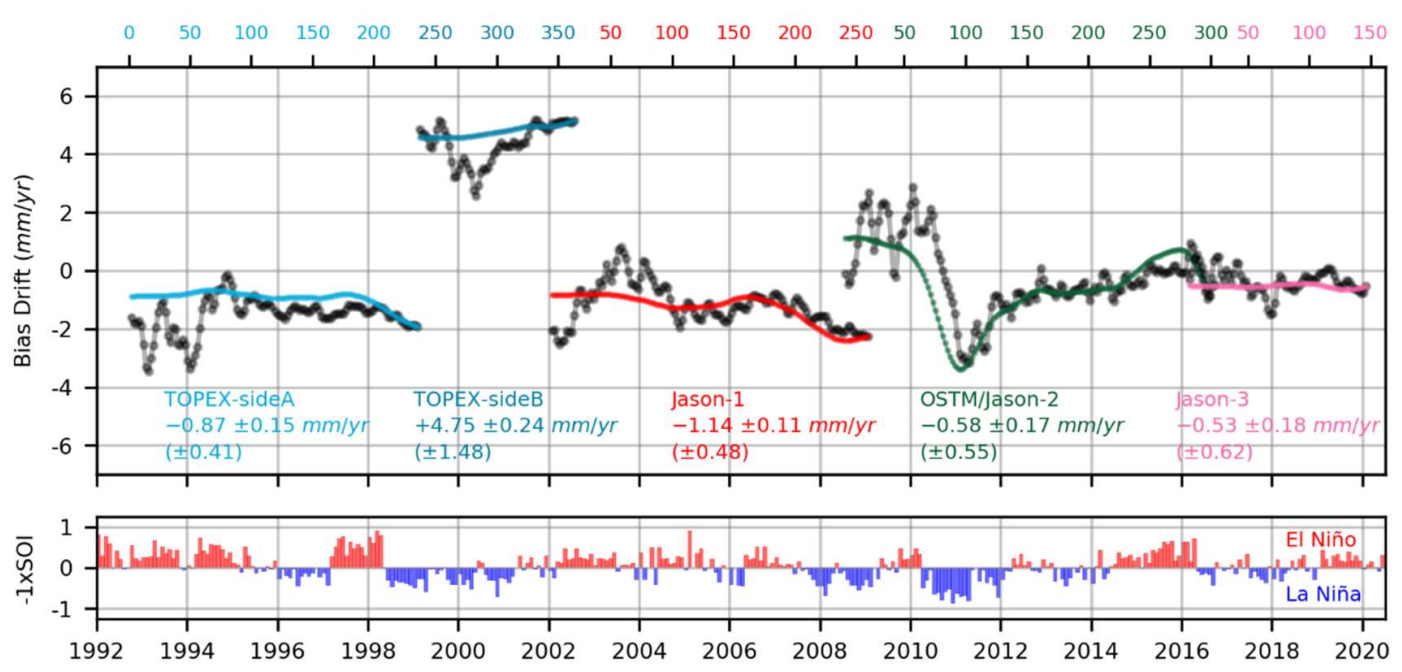

Figure S15. Estimated time variable systematic errors of the reference altimeters over the study region, in the solution when reference-mission data was only used. Comparing this with Figure 5 reveals a similar pattern of time-variability for Jason-2 drift. The mission-specific averages of smoothed bias drifts with the 1-sigma uncertainties are annotated, and the filter-based uncertainties are given in brackets. The sign-inversed Southern Oscillation Index (SOI) is shown in the lower panel.
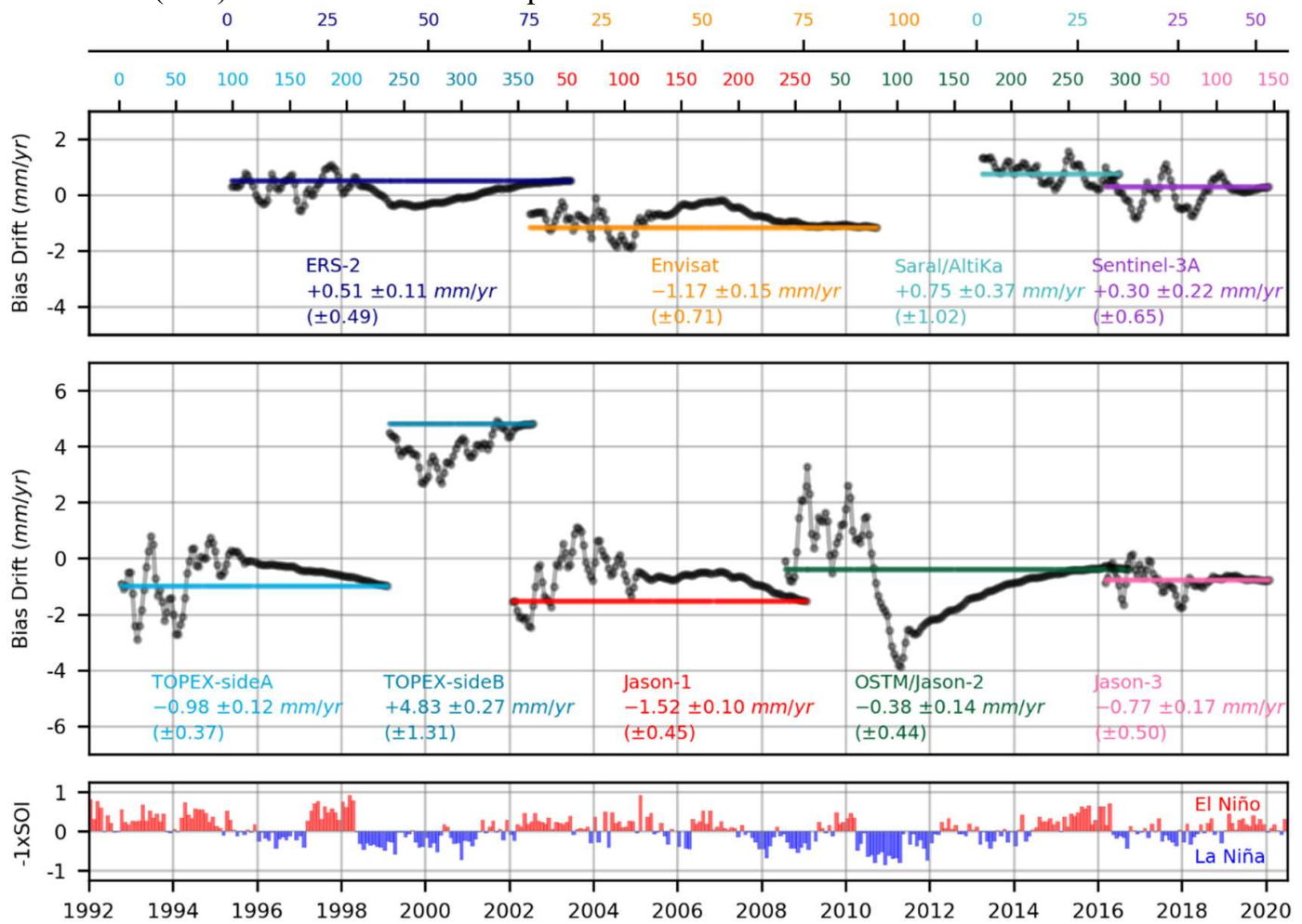

Figure S16. Estimated time-fixed systematic errors of each altimeter mission over the study region, from the solution when bias drifts were estimated as linear quantities with time. The mission-specific averages of smoothed bias drifts are annotated with the 1-sigma uncertainties that have been scaled by the a posteriori variance factor. The filtering uncertainties are given in brackets. The sign-inversed Southern Oscillation Index (SOI) is shown in the lower panel. 

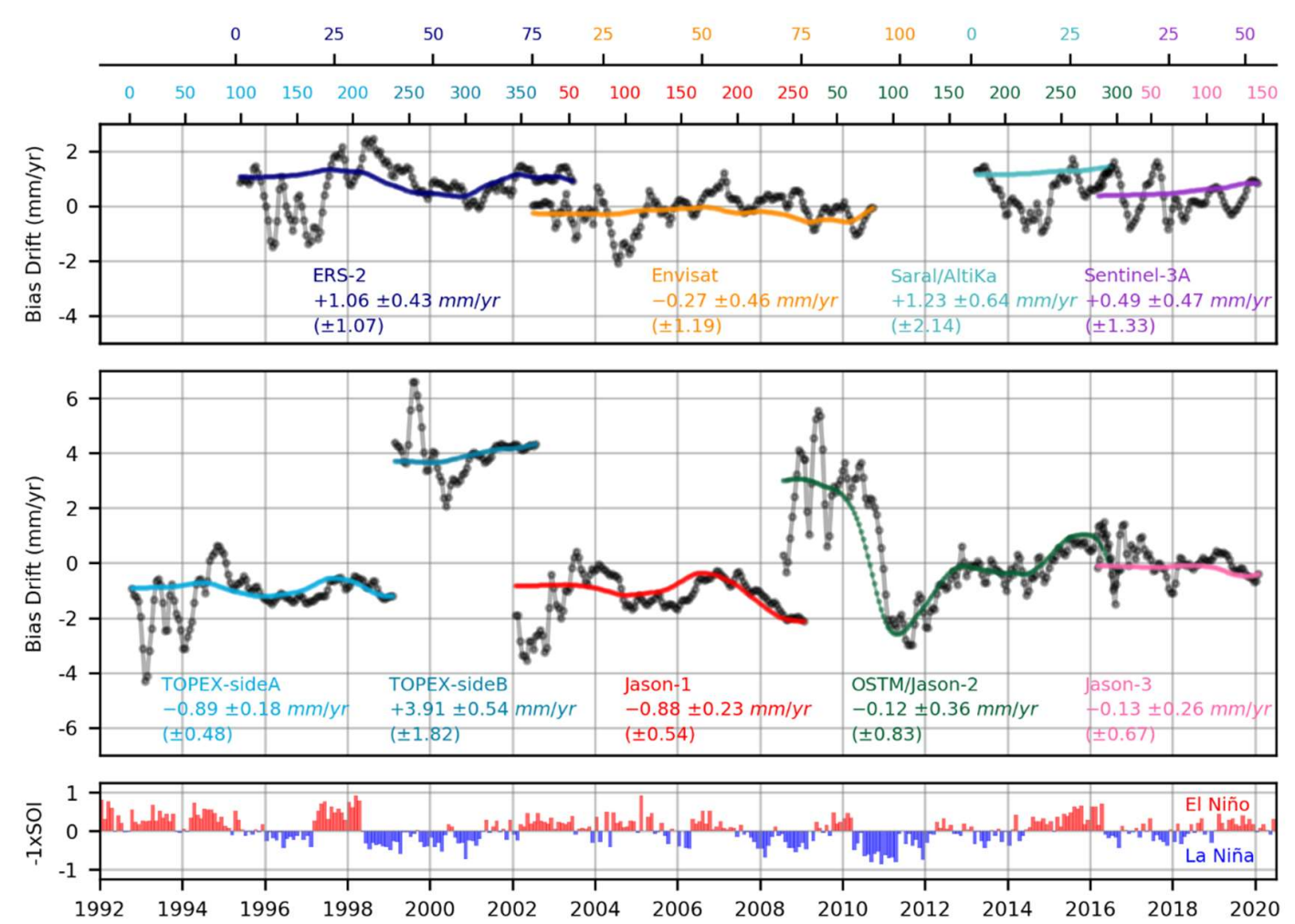

Figure S17. Mission-specific bias drifts in the solution when non-linear TG VLMs constrained to be zero at all geodetic sites. Note an anomalous variability in the case of Jason-2 mission, compared with Figure 5. The mission-specific averages of smoothed bias drifts are annotated with the 1-sigma uncertainties that have been scaled by the a posteriori variance factor. The filtering uncertainties are given in brackets. The sign-inversed Southern Oscillation Index (SOI) is shown in the lower panel.
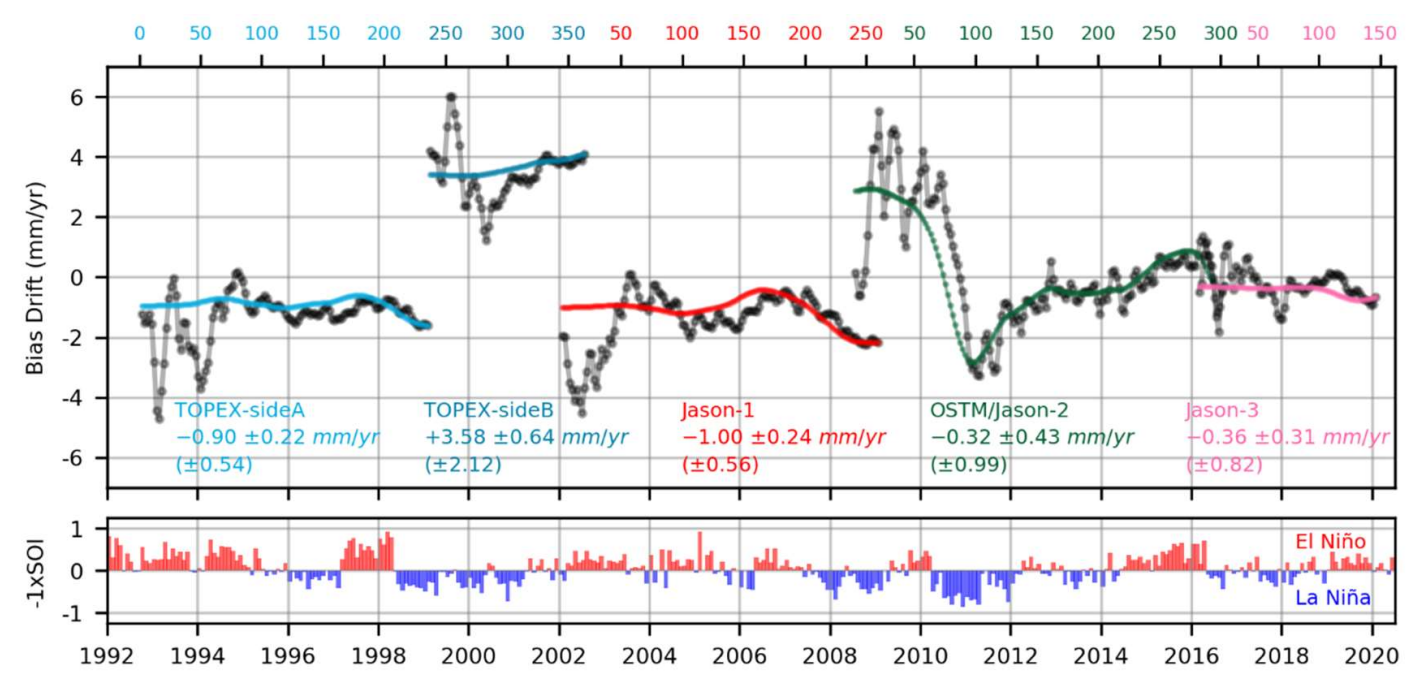

Figure S18. Mission-specific bias drifts in the solution when non-linear TG VLMs constrained to be zero at all geodetic sites, and only reference-mission data used. Note an anomalous variability in the case of Jason-2 mission, compared with Figure 5. The mission-specific averages of smoothed bias drifts are annotated with the 1-sigma uncertainties that have been scaled by the a posteriori variance factor. The filtering uncertainties are given in brackets. The sign-inversed Southern Oscillation Index (SOI) is shown in the lower panel. 

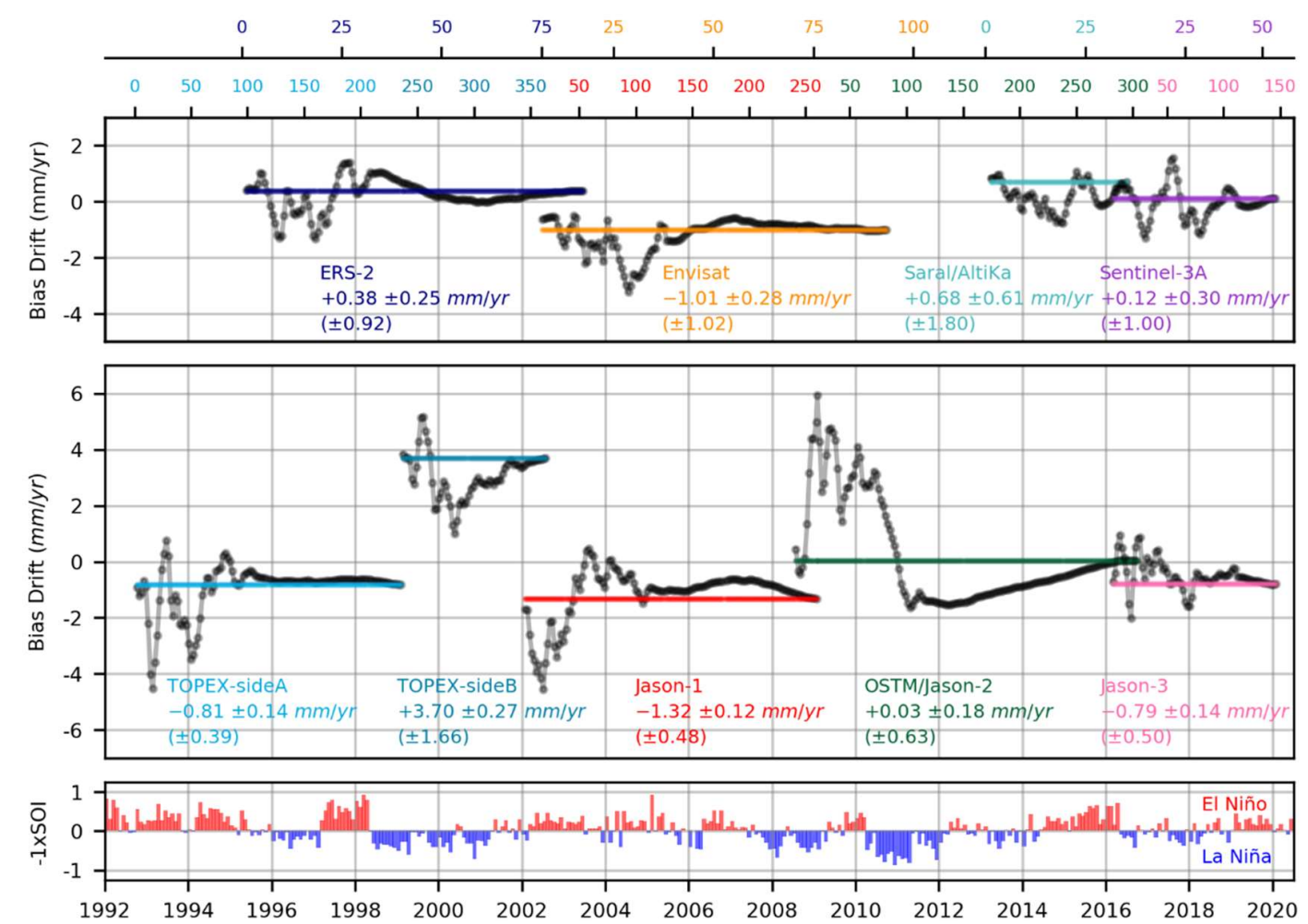

Figure S19. Mission-specific bias drifts in the solution when non-linear TG VLMs constrained to be zero at all geodetic sites, as assuming systematic errors in altimetry are behaving linearly in time. The mission-specific averages of smoothed bias drifts are annotated with the 1-sigma uncertainties that have been scaled by the a posteriori variance factor. The filtering uncertainties are given in brackets. The sign-inversed Southern Oscillation Index (SOI) is shown in the lower panel.
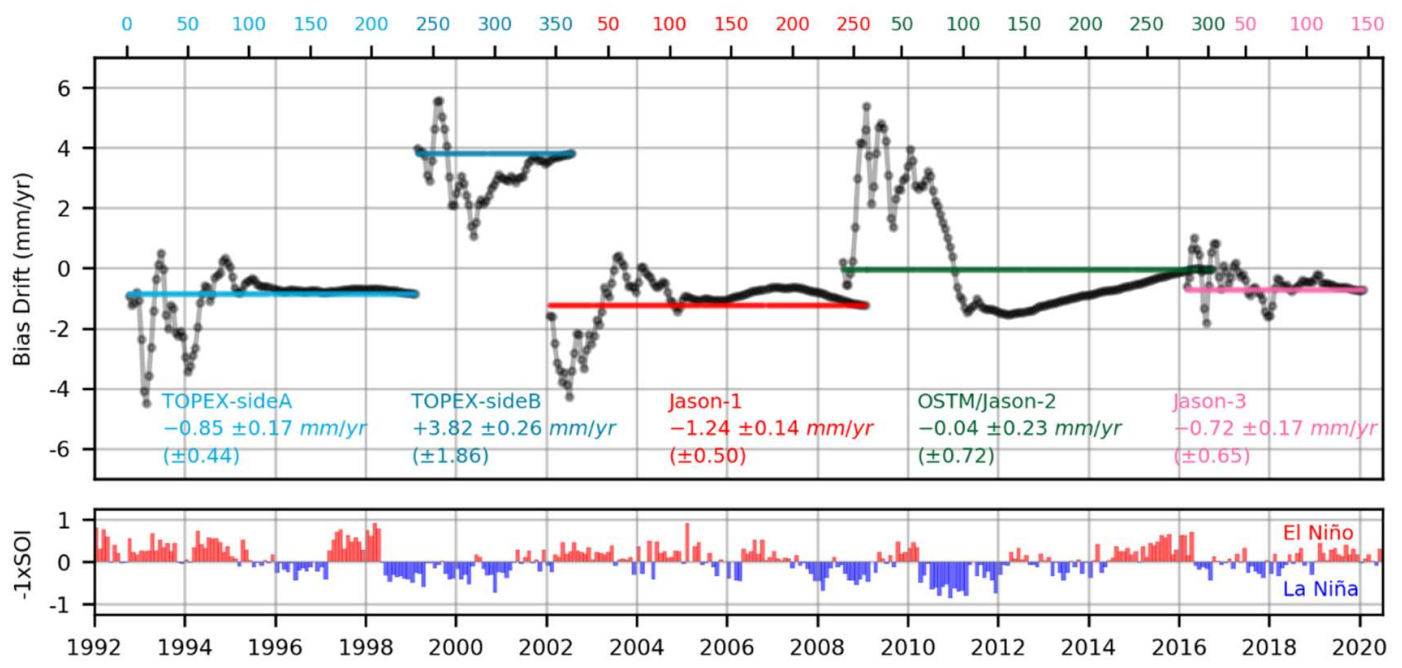

Figure S20. Mission-specific bias drifts in the solution when non-linear TG VLMs constrained to be zero at all geodetic sites, as assuming systematic errors in altimetry are behaving linearly in time, and only referencemission data used. The mission-specific averages of smoothed bias drifts are annotated with the 1-sigma uncertainties that have been scaled by the a posteriori variance factor. The filtering uncertainties are given in brackets. The sign-inversed Southern Oscillation Index (SOI) is shown in the lower panel. 


\subsection{Relative bias estimates}
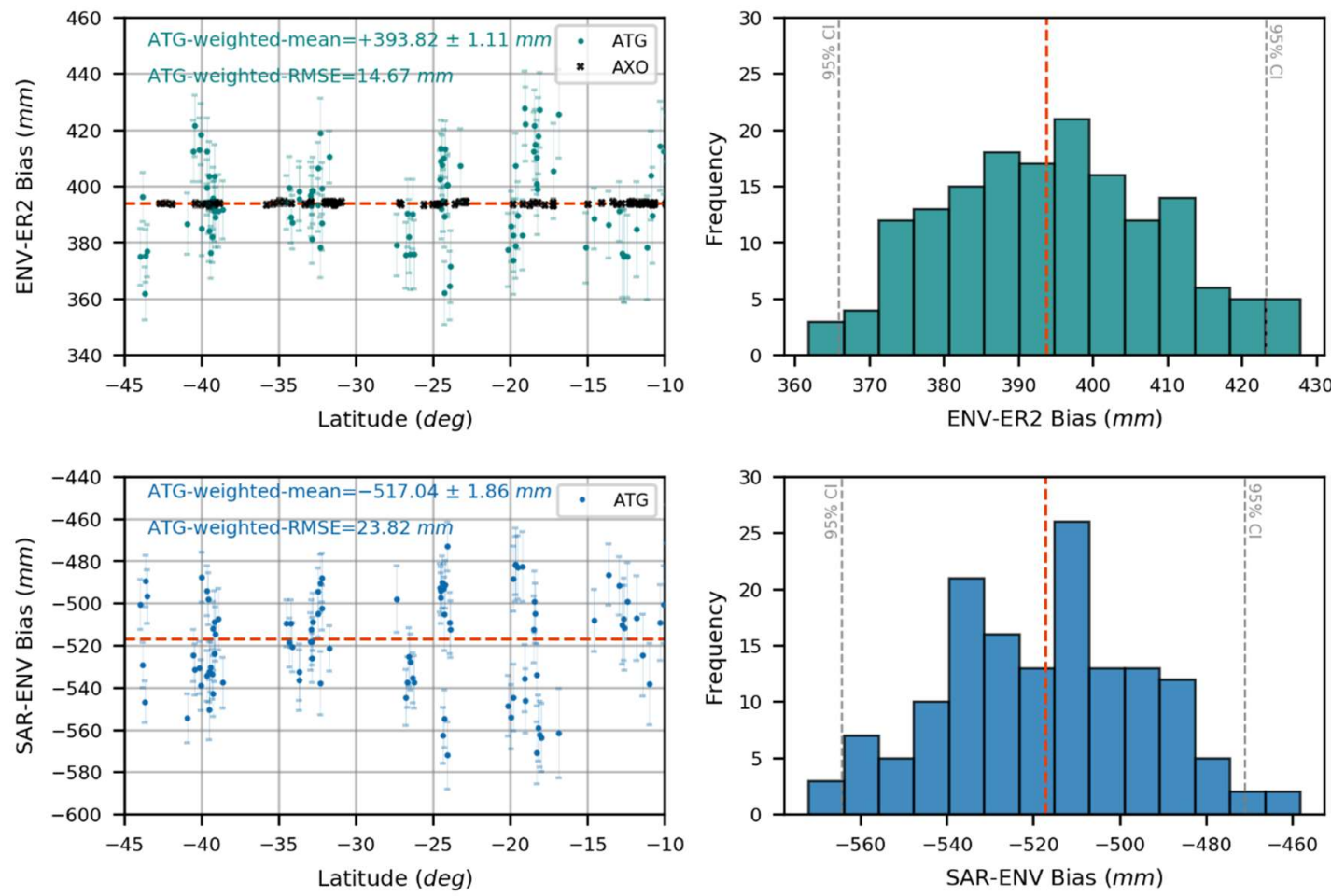

Figure S21. Intra and inter-mission relative biases of (top row) Envisat (ENV) minus ERS-2 (ER2), and (bottom row) SARAL (SAR) minus Envisat (ENV) at altimetry CPs derived from estimated intercepts of altimeter minus tide gauge (ATG) and tandem altimeter crossover (AXO) observations. Left panels compare profiles of ATG and AXO biases by latitude, and right panels show histograms of the ATG-only relative biases. The relative biases from the AXO tandem intercepts are shown with black crosses in the left panels (absent in the bottom row given no formation flight between ENV and SAR). The orange dashed lines show the most probable values of the intra- and inter-mission biases. Note the different scales on yaxes. 

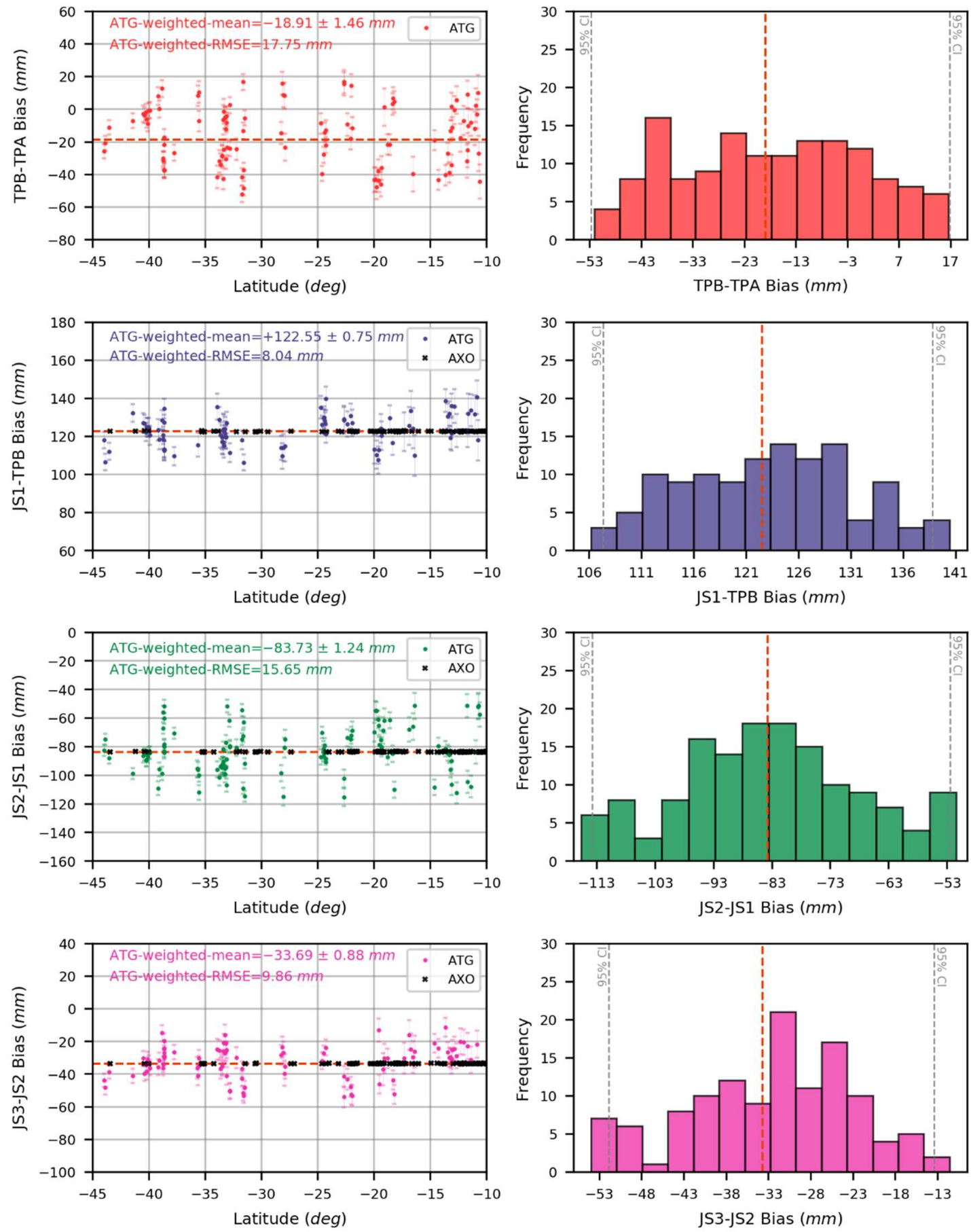

Figure S22. Intra and inter-mission relative biases of (top row) TOPEX-side B (TPB) minus TOPEX-side A (TPA), (second row) Jason-1 (JS1) minus TOPEX-side B (TPB), (third row) Jason-2 (JS2) minus Jason-1 (JS1), and (bottom row) Jason-3 (JS3) minus Jason-2 (JS2) at altimetry CPs derived from estimated intercepts of altimeter minus tide gauge (ATG) and tandem altimeter crossover (AXO) observations. Left panels compare profiles of ATG and AXO biases by latitude, and right panels show histograms of the ATG-only relative biases. The relative biases from the AXO tandem intercepts are shown with black crosses in the left panels (absent in the top row given no formation flight between TPA and TPB). The orange dashed lines show the most probable values of the intra- and inter-mission biases. Note the different scales on y-axes. 




(a) GPS-Krig ASL estimate

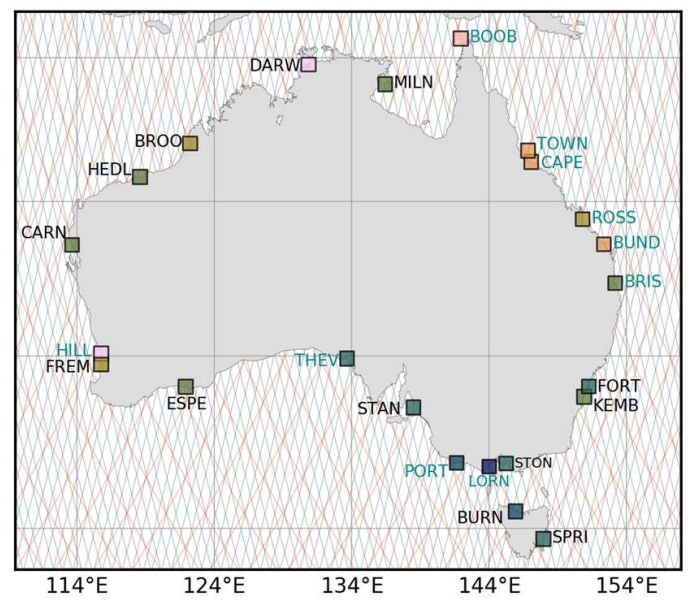

(b) GIA ASL estimate

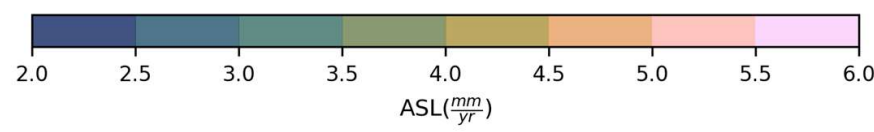

Figure S23. Map of ASL at TG locations resulted by applying linear VLMs from (a) GPS-Krig, and (b) ICE6G_D model to the RSL trends in the same timeframe. TGs with 1-sigma significant differences of our VLMs minus GPS-Krig and GIA are annotated in green and cyan in the left and right, respectively. For clarity, TG latitude at TOWN and FREM locations are shifted by +0.75 and -0.45 degrees, respectively. The ground tracks of Jason-series and Envisat-series altimeters are shown in orange and cyan, respectively.

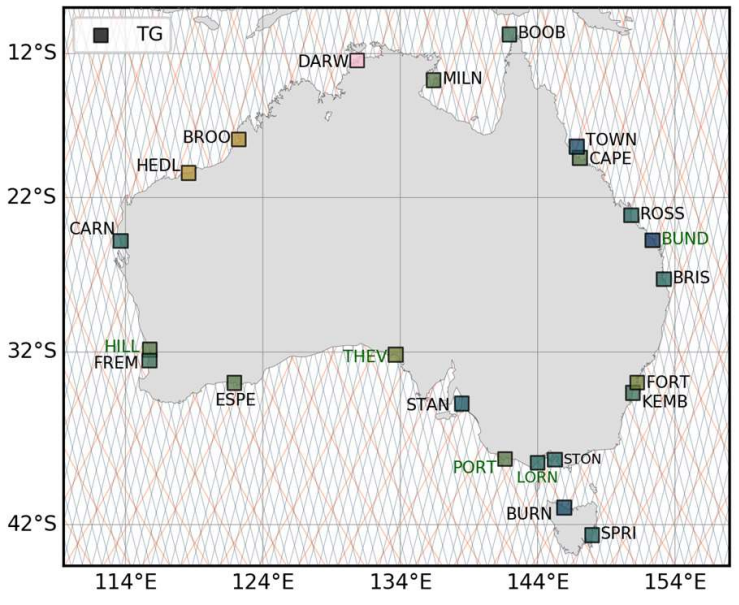

(a) Our Ref-mission ASL estimate

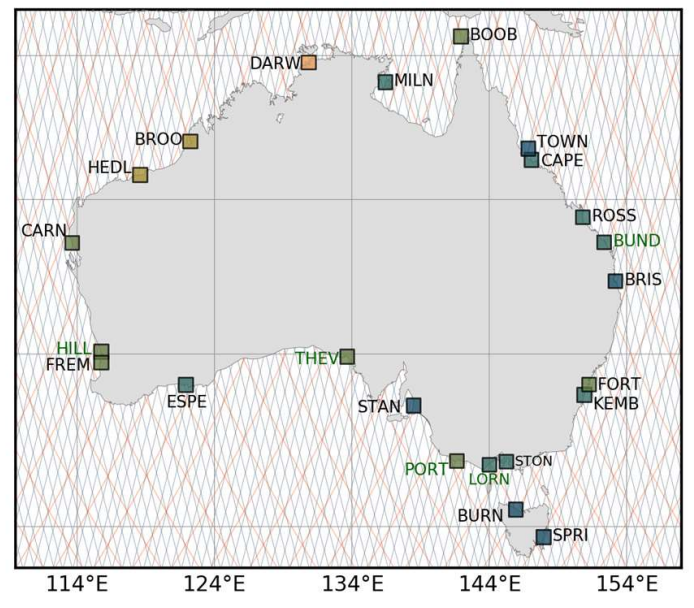

(b) Our Multi-mission ASL estimate

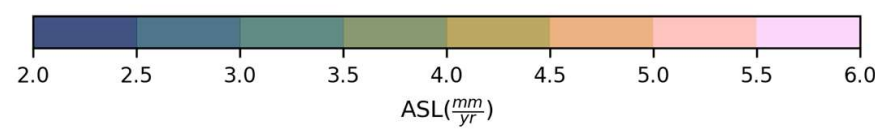

Figure S24. Map of ASL at TG locations resulted by (a) reference-mission and (b) multi-mission and solutions. TGs with 1-sigma significant differences of our VLMs minus GPS-Krig and GIA are annotated in green and cyan in the left and right, respectively. For clarity, TG latitude at TOWN and FREM locations are shifted by +0.75 and -0.45 degrees, respectively. The ground tracks of Jason-series and Envisat-series altimeters are shown in orange and cyan, respectively. 


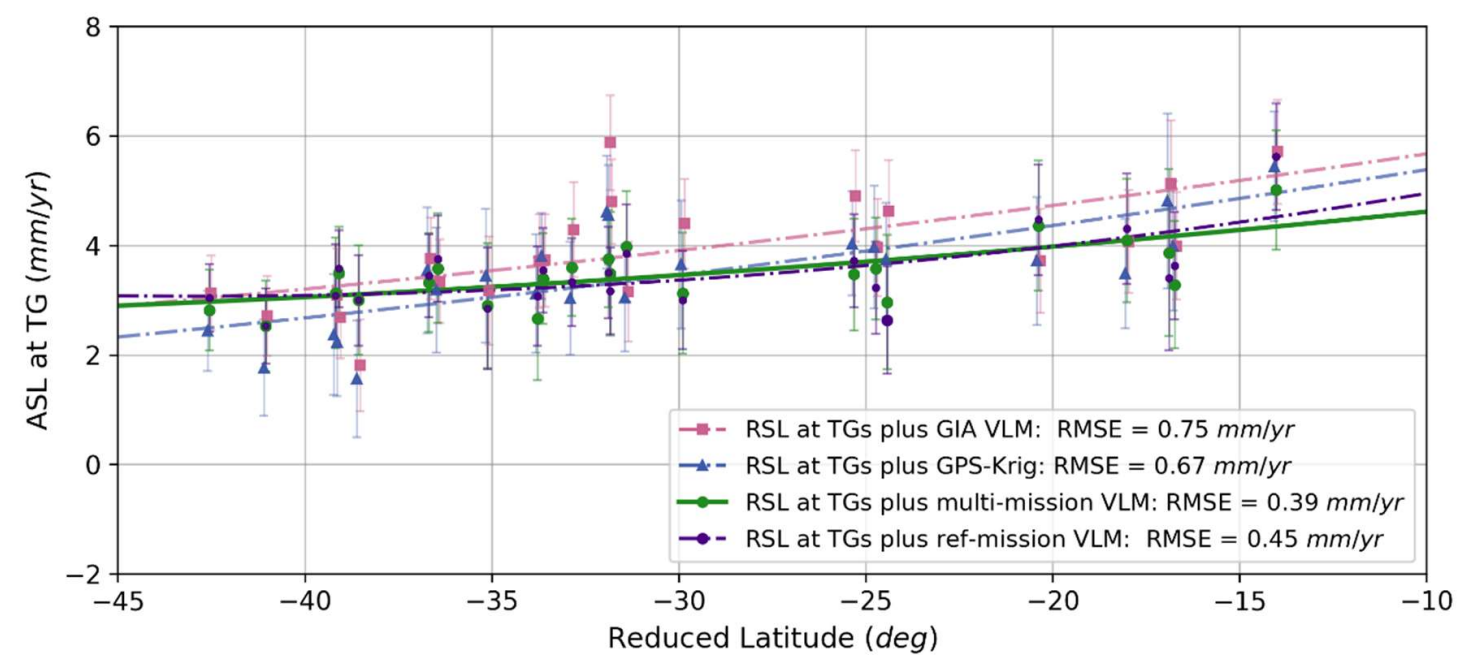

Figure S25. Profile of ASL trends at TGs as function of latitude, comparing estimates derived using our ref-mission VLM (purple circles), multi-mission VLM (green circles) with GPS-Krig (blue triangles) and ICE6G_D GIA (pink squares). Solid and dashed lines show a quadratic polynomial fitted to each set of ASL estimates per reduced latitude to the SE-NW direction with RMSE about this fit annotated in the legend. For clarity, TG latitudes at STON and CARN locations are shifted by -0.75 degrees, and TG latitudes at PORT, ESPE, HILL, THEV and TOWN are shifted by +0.75 degrees. The latitudes of TGs where ASL is derived from GIA and GPS-Krig are also shifted by +0.045 and -0.045 degrees, respectively. Error bars are \pm 1 -sigma scaled by the a posteriori variance factor.

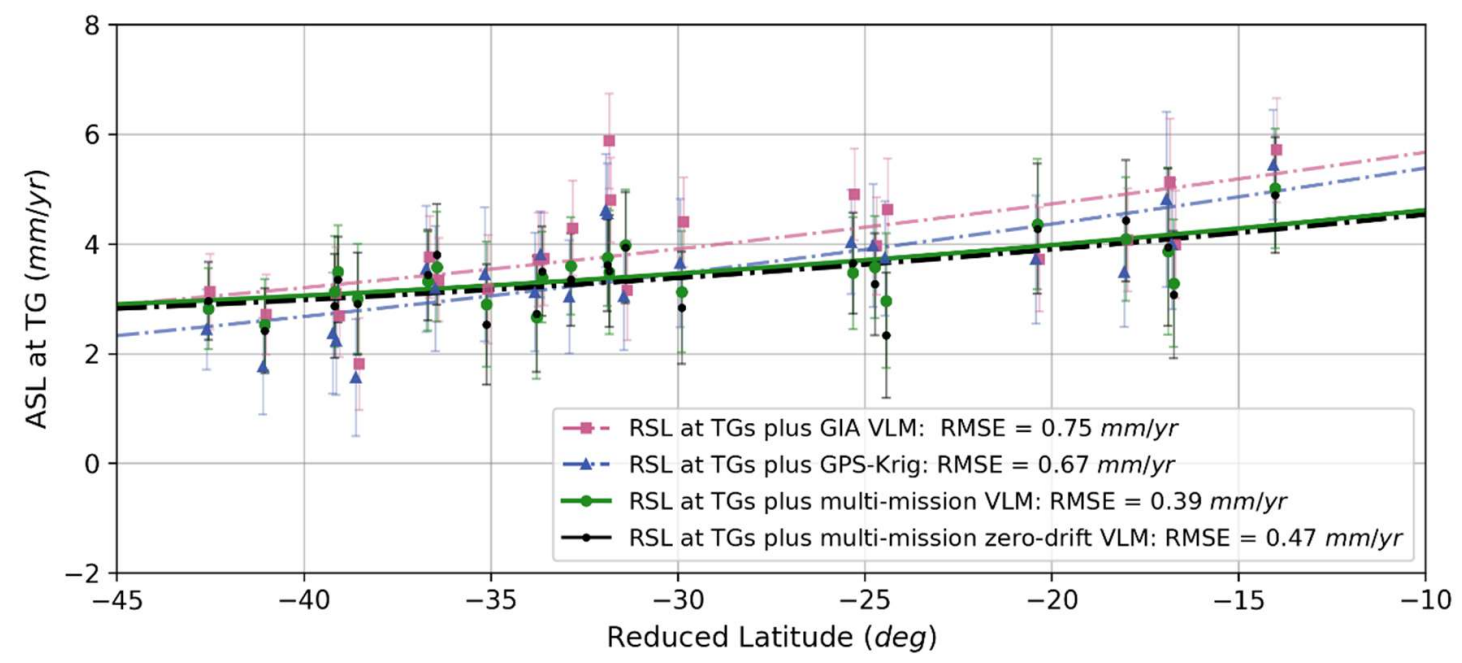

Figure S26. Profile of ASL trends at TGs as function of latitude, comparing estimates derived using our 'zero-drift' multi-mission VLM (black circles), multi-mission VLM (green circles) with GPS-Krig (blue triangles) and ICE6G_D GIA (pink squares). Solid and dashed lines show a quadratic polynomial fitted to each set of ASL estimates per latitude reduced to the SE-NW direction with RMSE about this fit annotated in the legend. For clarity, TG latitudes at STON and CARN locations are shifted by -0.75 degrees, and TG latitudes at PORT, ESPE, HILL, THEV and TOWN are shifted by +0.75 degrees. The latitudes of TGs where ASL is derived from GIA and GPS-Krig are also shifted by +0.045 and -0.045 degrees, respectively. Error bars are \pm 1 -sigma scaled by the a posteriori variance factor. 
manuscript submitted to Journal of Geodesy

1605

1606

Table S4. GPS-Krig and GIA trends versus our a posteriori VLMs at TG locations, along with RSL and our ASL estimates. Note the timespan and \pm 1 -sigma uncertainties.

\begin{tabular}{|c|c|c|c|c|c|c|c|c|}
\hline TG name & $\begin{array}{c}\text { Lat } \\
\text { (deg) }\end{array}$ & $\begin{array}{c}\text { Lon } \\
\text { (deg) }\end{array}$ & $\begin{array}{l}\text { Time } \\
\text { span }\end{array}$ & $\begin{array}{c}\text { GPS-Krig } \\
\text { VLM } \\
(\mathrm{mm} / \mathrm{yr}) \\
\end{array}$ & $\begin{array}{l}\text { GIA VLM } \\
\text { (mm/yr) }\end{array}$ & $\begin{array}{c}\text { Our VLM } \\
(\mathrm{mm} / \mathbf{y r})\end{array}$ & $\begin{array}{l}\text { RSL estimates } \\
(\mathrm{mm} / \mathrm{yr})\end{array}$ & $\begin{array}{l}\text { Our ASL } \\
(\mathbf{m m} / \mathbf{y r})\end{array}$ \\
\hline BOOBY_ISL(BOOB) & -10.6 & 141.92 & $\begin{array}{l}1992.7 \\
2019.8\end{array}$ & $1.07 \pm 1.94$ & $-0.06 \pm 1.0$ & $-1.32 \pm 1.02$ & $6.09 \pm 1.03$ & $4.77 \pm 1.45$ \\
\hline BRISBANE(BRIS) & -27.37 & 153.17 & \begin{tabular}{|l}
1992.8 \\
2020.1 \\
\end{tabular} & $-0.74 \pm 0.94$ & $-0.14 \pm 1.0$ & $-1.19 \pm 0.82$ & $3.99 \pm 0.37$ & $2.80 \pm 0.90$ \\
\hline BROOME(BROO) & -18.0 & 122.22 & $\begin{array}{l}1992.8 \\
2020.1 \\
\end{array}$ & $-0.81 \pm 0.7$ & $-0.22 \pm 1.0$ & $-0.2 \pm 0.76$ & $4.15 \pm 0.56$ & $3.94 \pm 0.94$ \\
\hline BUNDABERG(BUND) & -24.83 & 152.35 & $\begin{array}{l}1992.7 \\
2019.0\end{array}$ & $-0.37 \pm 0.8$ & $-0.13 \pm 1.0$ & $-1.45 \pm 0.9$ & $5.33 \pm 0.38$ & $3.88 \pm 0.98$ \\
\hline BURNIE(BURN) & -41.05 & 145.92 & $\begin{array}{l}1992.7 \\
2020.1\end{array}$ & $-1.17 \pm 0.8$ & $-0.21 \pm 1.0$ & $-0.4 \pm 0.61$ & $3.16 \pm 0.27$ & $2.76 \pm 0.67$ \\
\hline CAPE_FERGUSON(CAPE) & -19.28 & 147.06 & $\begin{array}{l}1992.7 \\
2020.1 \\
\end{array}$ & $-0.98 \pm 0.78$ & $-0.11 \pm 1.0$ & $-1.54 \pm 0.74$ & $5.55 \pm 0.32$ & $4.01 \pm 0.8$ \\
\hline CARNARVON(CARN) & -24.88 & 113.62 & $\begin{array}{l}1992.7 \\
2020.1\end{array}$ & $-0.27 \pm 0.79$ & $-0.27 \pm 1.0$ & $-0.66 \pm 0.57$ & $4.45 \pm 0.64$ & $3.79 \pm 0.86$ \\
\hline DARWIN(DARW) & -12.47 & 130.85 & $\begin{array}{l}1992.7 \\
2020.0 \\
\end{array}$ & $-0.34 \pm 0.69$ & $-0.07 \pm 1.0$ & $-0.77 \pm 0.7$ & $5.28 \pm 0.6$ & $4.51 \pm 0.92$ \\
\hline ESPERANCE(ESPE) & -33.87 & 121.9 & $\begin{array}{l}1992.7 \\
2020.0\end{array}$ & $-0.26 \pm 0.49$ & $-0.34 \pm 1.0$ & $-0.67 \pm 0.47$ & $3.85 \pm 0.43$ & $3.18 \pm 0.64$ \\
\hline FORT_DENISON(FORT) & -33.85 & 151.23 & $\begin{array}{l}1992.7 \\
2020.0\end{array}$ & $-0.38 \pm 0.78$ & $-0.2 \pm 1.0$ & $0.04 \pm 0.77$ & $3.43 \pm 0.3$ & $3.47 \pm 0.83$ \\
\hline FREMANTLE(FREM) & -32.05 & 115.73 & $\begin{array}{l}1992.7 \\
2020.1\end{array}$ & $-1.33 \pm 0.73$ & $-0.27 \pm 1.0$ & $-0.96 \pm 0.53$ & $4.38 \pm 0.56$ & $3.42 \pm 0.77$ \\
\hline HEDLAND(HEDL) & -20.32 & 118.57 & $\begin{array}{l}1992.7 \\
2019.2\end{array}$ & $-0.24 \pm 0.79$ & $-0.24 \pm 1.0$ & $0.4 \pm 0.82$ & $4.05 \pm 0.60$ & $4.45 \pm 1.02$ \\
\hline HILLARYS(HILL) & -31.83 & 115.74 & $\begin{array}{l}1992.7 \\
2020.1\end{array}$ & $-1.33 \pm 0.72$ & $-0.26 \pm 1.0$ & $-2.38 \pm 0.52$ & $5.82 \pm 0.58$ & $3.44 \pm 0.78$ \\
\hline KEMBLA(KEMB) & -34.47 & 150.91 & $\begin{array}{l}1992.7 \\
2020.1\end{array}$ & $-0.4 \pm 0.81$ & $-0.2 \pm 1.0$ & $-0.64 \pm 0.68$ & $3.84 \pm 0.28$ & $3.2 \pm 0.73$ \\
\hline LORNE(LORN) & -38.55 & 143.99 & $\begin{array}{l}1993.0 \\
2020.1 \\
\end{array}$ & $-0.49 \pm 0.94$ & $-0.25 \pm 1.0$ & $0.95 \pm 0.71$ & $2.01 \pm 0.35$ & $2.96 \pm 0.79$ \\
\hline MILNER_BAY(MILN) & -13.86 & 136.42 & $\begin{array}{l}1993.7 \\
2020.0\end{array}$ & $-0.14 \pm 0.79$ & $-0.14 \pm 1.0$ & $-0.85 \pm 0.76$ & $4.90 \pm 0.84$ & $4.05 \pm 1.13$ \\
\hline PORTLAND(PORT) & -38.34 & 141.61 & $\begin{array}{l}1992.7 \\
2020.1\end{array}$ & $-0.78 \pm 0.92$ & $-0.33 \pm 1.0$ & $0.49 \pm 0.6$ & $2.81 \pm 0.33$ & $3.30 \pm 0.68$ \\
\hline ROSSLYN_BAY(ROSS) & -23.16 & 150.79 & $\begin{array}{l}1993.2 \\
2020.1\end{array}$ & $-0.63 \pm 1.17$ & $-0.07 \pm 1.0$ & $-1.34 \pm 0.84$ & $4.59 \pm 0.3$ & $3.25 \pm 0.9$ \\
\hline SPRING_BAY(SPRI) & -42.55 & 147.93 & $\begin{array}{l}1992.7 \\
2020.0\end{array}$ & $-0.88 \pm 0.66$ & $-0.19 \pm 1.0$ & $-0.5 \pm 0.56$ & $3.39 \pm 0.23$ & $2.89 \pm 0.6$ \\
\hline STANVAC(STAN) & -35.11 & 138.47 & $\begin{array}{l}1992.7 \\
2009.1 \\
\end{array}$ & $-0.25 \pm 1.07$ & $-0.25 \pm 1.0$ & $-0.52 \pm 0.71$ & $5.15 \pm 0.81$ & $4.63 \pm 1.08$ \\
\hline STONY_POINT(STON) & -38.37 & 145.22 & \begin{tabular}{|l}
1993.0 \\
2010.8 \\
\end{tabular} & $-0.8 \pm 1.01$ & $-0.19 \pm 1.0$ & $-0.15 \pm 0.7$ & $2.92 \pm 0.42$ & $2.77 \pm 0.82$ \\
\hline THEVENARD(THEV) & -32.15 & 133.64 & \begin{tabular}{|l}
1992.7 \\
2019.4 \\
\end{tabular} & $-0.39 \pm 0.7$ & $-0.28 \pm 1.0$ & $0.54 \pm 0.64$ & $3.65 \pm 0.44$ & $4.19 \pm 0.78$ \\
\hline TOWNSVILLE(TOWN) & -19.25 & 146.83 & $\begin{array}{l}1992.7 \\
2016.7\end{array}$ & $-0.98 \pm 0.78$ & $-0.1 \pm 1.0$ & $-1.75 \pm 0.88$ & $4.65 \pm 0.56$ & $2.90 \pm 1.04$ \\
\hline
\end{tabular}


manuscript submitted to Journal of Geodesy

1612 Table S5. Our a posteriori VLMs at GPS sites against the Hector-derived and ICE6G_D trends. Note the 1613 timespan of observational records. All uncertainties are given at \pm 1 -sigma level.

\begin{tabular}{|c|c|c|c|c|c|c|c|c|c|c|c|c|c|}
\hline $\begin{array}{c}\text { GPS } \\
\text { name }\end{array}$ & $\begin{array}{l}\text { Lat } \\
\text { (de) }\end{array}$ & $\begin{array}{c}\text { Lon } \\
\text { (deg) }\end{array}$ & $\begin{array}{l}\text { Time } \\
\text { Span }\end{array}$ & $\begin{array}{c}\text { Hector } \\
\text { VLM } \\
(\mathrm{mm} / \mathrm{yr})\end{array}$ & $\begin{array}{c}\text { GIA VLM } \\
\text { (mm/yr) }\end{array}$ & $\underset{(\mathbf{m m} / \mathbf{y r})}{\text { Our VLM }}$ & $\begin{array}{c}\text { GPS } \\
\text { name }\end{array}$ & $\begin{array}{c}\text { Lat } \\
\text { (deg) }\end{array}$ & $\begin{array}{l}\text { Lon } \\
\text { (deg) }\end{array}$ & $\begin{array}{l}\text { Time } \\
\text { span }\end{array}$ & $\begin{array}{c}\text { Hector } \\
\text { VLM } \\
(\mathbf{m m} / \mathbf{y r})\end{array}$ & $\begin{array}{c}\text { GIA VLM } \\
(\mathrm{mm} / \mathrm{yr})\end{array}$ & $\begin{array}{c}\text { Our VLM } \\
(\mathbf{m m} / \mathbf{y r})\end{array}$ \\
\hline $00 \mathrm{NA}$ & -12.47 & 130.84 & $\begin{array}{l}2008.2 \\
2018.7 \\
\end{array}$ & $-1.7 \pm 0.59$ & $-0.07 \pm 1.0$ & $-1.52 \pm 0.53$ & CBTN & -34.06 & 150.82 & $\begin{array}{l}2012.2 \\
2019.7 \\
\end{array}$ & $-1.77 \pm 0.97$ & $-0.16 \pm 1.0$ & $-1.54 \pm 0.58$ \\
\hline $4 \mathrm{CDA}$ & -28.24 & 153.56 & $\begin{array}{l}2012.2 \\
2015.9\end{array}$ & $1.86 \pm 1.38$ & $-0.19 \pm 1.0$ & $2.1 \pm 1.52$ & CEDU & -31.87 & 133.81 & $\begin{array}{l}1994.4 \\
2019.7\end{array}$ & $-0.45 \pm 0.34$ & $-0.22 \pm 1.0$ & $-0.39 \pm 0.48$ \\
\hline A770 & -24.16 & 151.89 & $\begin{array}{l}2014.0 \\
2019.7 \\
\end{array}$ & $1.68 \pm 0.51$ & $-0.12 \pm 1.0$ & $1.73 \pm 0.62$ & CLEV & -27.53 & 153.27 & $\begin{array}{l}2009.2 \\
2019.7 \\
\end{array}$ & $-0.7 \pm 0.67$ & $-0.15 \pm 1.0$ & $-0.64 \pm 0.5$ \\
\hline ACA4 & -27.6 & 153.04 & $\begin{array}{l}2012.8 \\
2018.9\end{array}$ & $0.24 \pm 1.0$ & $-0.11 \pm 1.0$ & $0.31 \pm 0.67$ & CNLP & -35.69 & 139.85 & $\begin{array}{l}2010.7 \\
2019.6 \\
\end{array}$ & $-0.56 \pm 0.69$ & $-0.17 \pm 1.0$ & $-0.4 \pm 0.52$ \\
\hline ACL2 & -27.27 & 151.7 & $\begin{array}{l}2010.7 \\
2019.6 \\
\end{array}$ & $-1.92 \pm 0.79$ & $0.02 \pm 1.0$ & $-1.69 \pm 0.51$ & COEN & -13.96 & 143.18 & $\begin{array}{l}2013.5 \\
2019.7 \\
\end{array}$ & $0.12 \pm 1.1$ & $-0.0 \pm 1.0$ & $0.28 \pm 0.78$ \\
\hline ADE1 & -34.73 & 138.65 & $\begin{array}{l}1999.2 \\
2011.5\end{array}$ & $-0.89 \pm 0.5$ & $-0.2 \pm 1.0$ & $-1.04 \pm 0.41$ & COOR & -25.01 & 149.5 & $\begin{array}{l}2013.6 \\
2019.2 \\
\end{array}$ & $-0.66 \pm 0.76$ & $0.01 \pm 1.0$ & $-0.49 \pm 0.75$ \\
\hline ADE2 & -34.73 & 138.65 & $\begin{array}{l}2005.8 \\
2011.5\end{array}$ & $-1.5 \pm 1.04$ & $-0.2 \pm 1.0$ & $-1.74 \pm 0.78$ & $\mathrm{CRCW}$ & -25.29 & 150.27 & $\begin{array}{l}2013.6 \\
2019.7\end{array}$ & $-0.66 \pm 0.6$ & $0.02 \pm 1.0$ & $-0.61 \pm 0.72$ \\
\hline AMB1 & -41.2 & 146.38 & $\begin{array}{l}2013.6 \\
2019.6\end{array}$ & $-1.68 \pm 0.67$ & $-0.18 \pm 1.0$ & $-1.59 \pm 0.63$ & CRK1 & -33.92 & 151.18 & $\begin{array}{l}2010.7 \\
2015.5 \\
\end{array}$ & $0.4 \pm 0.81$ & $-0.2 \pm 1.0$ & $0.21 \pm 0.9$ \\
\hline ARRT & -37.28 & 142.93 & $\begin{array}{l}2013.4 \\
2019.6\end{array}$ & $-0.68 \pm 1.03$ & $-0.09 \pm 1.0$ & $-0.48 \pm 0.62$ & CRKB & -27.44 & 153.05 & $\begin{array}{l}2010.7 \\
2019.7\end{array}$ & $-2.82 \pm 0.85$ & $-0.12 \pm 1.0$ & $-2.65 \pm 0.53$ \\
\hline BALA & -32.46 & 123.87 & $\begin{array}{l}2012.0 \\
2019.7\end{array}$ & $-0.34 \pm 0.92$ & $-0.21 \pm 1.0$ & $-0.41 \pm 0.57$ & CRKP & -31.94 & 115.84 & $\begin{array}{l}2010.7 \\
2018.1\end{array}$ & $-1.2 \pm 1.17$ & $-0.25 \pm 1.0$ & $-1.04 \pm 0.58$ \\
\hline BALL & -37.56 & 143.85 & $\begin{array}{l}2011.0 \\
2019.6\end{array}$ & $-0.1 \pm 0.72$ & $-0.1 \pm 1.0$ & $-0.1 \pm 0.51$ & CRL0 & -27.54 & 153.42 & $\begin{array}{l}2010.7 \\
2019.7\end{array}$ & $-2.7 \pm 0.82$ & $-0.18 \pm 1.0$ & $-2.53 \pm 0.56$ \\
\hline BALM & -37.25 & 141.84 & \begin{tabular}{|l|}
2013.4 \\
2019.6 \\
\end{tabular} & $-0.83 \pm 0.74$ & $-0.14 \pm 1.0$ & $-0.62 \pm 0.59$ & CRY4 & -26.42 & 152.91 & $\begin{array}{l}2012.0 \\
2018.9 \\
\end{array}$ & $-1.48 \pm 1.15$ & $-0.13 \pm 1.0$ & $-1.6 \pm 0.65$ \\
\hline BANK & -33.92 & 151.04 & $\begin{array}{l}2014.1 \\
2019.6 \\
\end{array}$ & $-0.18 \pm 0.93$ & $-0.18 \pm 1.0$ & $-0.13 \pm 0.71$ & CUAA & -32.0 & 115.89 & $\begin{array}{l}2012.8 \\
2019.7 \\
\end{array}$ & $-1.18 \pm 0.91$ & $-0.24 \pm 1.0$ & $-1.31 \pm 0.6$ \\
\hline BAT2 & -33.43 & 149.57 & $\begin{array}{l}2010.9 \\
2019.6 \\
\end{array}$ & $-0.24 \pm 0.72$ & $-0.03 \pm 1.0$ & $-0.17 \pm 0.55$ & CUAI & -32.0 & 115.89 & $\begin{array}{l}2012.8 \\
2019.7 \\
\end{array}$ & $-1.12 \pm 0.87$ & $-0.24 \pm 1.0$ & $-1.11 \pm 0.6$ \\
\hline BBOO & -32.81 & 136.06 & $\begin{array}{l}2009.6 \\
2019.7 \\
\end{array}$ & $-0.46 \pm 0.62$ & $-0.16 \pm 1.0$ & $-0.36 \pm 0.49$ & CUBB & -32.0 & 115.89 & $\begin{array}{l}2012.7 \\
2019.7 \\
\end{array}$ & $-1.24 \pm 0.94$ & $-0.24 \pm 1.0$ & $-1.14 \pm 0.61$ \\
\hline BCMT & -28.13 & 153.19 & $\begin{array}{l}2010.7 \\
2019.0\end{array}$ & $0.72 \pm 0.86$ & $-0.13 \pm 1.0$ & $0.68 \pm 0.54$ & CUC2 & -32.0 & 115.89 & $\begin{array}{l}2015.2 \\
2019.7 \\
\end{array}$ & $-2.04 \pm 1.32$ & $-0.24 \pm 1.0$ & $-2.12 \pm 0.93$ \\
\hline BDLE & -37.76 & 147.66 & $\begin{array}{l}2009.7 \\
2019.7\end{array}$ & $-0.78 \pm 0.66$ & $-0.13 \pm 1.0$ & $-1.0 \pm 0.51$ & CUT0 & -32.0 & 115.89 & $\begin{array}{l}2010.6 \\
2019.7 \\
\end{array}$ & $-1.66 \pm 0.57$ & $-0.24 \pm 1.0$ & $-1.81 \pm 0.51$ \\
\hline BDRM & -26.68 & 153.07 & $\begin{array}{l}2010.7 \\
2019.0\end{array}$ & $-0.26 \pm 0.84$ & $-0.14 \pm 1.0$ & $-0.18 \pm 0.54$ & CUT1 & -32.0 & 115.89 & $\begin{array}{l}2010.5 \\
2019.0\end{array}$ & $-2.03 \pm 0.69$ & $-0.24 \pm 1.0$ & $-2.18 \pm 0.54$ \\
\hline BDST & -27.99 & 153.0 & $\begin{array}{l}2009.2 \\
2019.7\end{array}$ & $-0.8 \pm 0.43$ & $-0.1 \pm 1.0$ & $-0.89 \pm 0.51$ & CUT3 & -32.0 & 115.89 & $\begin{array}{l}2012.4 \\
2019.7\end{array}$ & $-1.52 \pm 0.81$ & $-0.24 \pm 1.0$ & $-1.59 \pm 0.63$ \\
\hline BER5 & -34.28 & 140.6 & $\begin{array}{l}2014.5 \\
2019.7\end{array}$ & $-0.2 \pm 0.84$ & $-0.08 \pm 1.0$ & $-0.28 \pm 0.71$ & DALB & -27.17 & 151.26 & $\begin{array}{l}2010.6 \\
2019.7\end{array}$ & $-2.6 \pm 0.49$ & $0.03 \pm 1.0$ & $-2.66 \pm 0.52$ \\
\hline BIN2 & -32.41 & 151.65 & $\begin{array}{l}2012.0 \\
2015.8 \\
\end{array}$ & $1.74 \pm 1.08$ & $-0.12 \pm 1.0$ & $1.89 \pm 1.16$ & DARM & -12.42 & 130.89 & $\begin{array}{l}2007.0 \\
2014.8 \\
\end{array}$ & $-1.1 \pm 0.51$ & $-0.07 \pm 1.0$ & $-1.14 \pm 0.56$ \\
\hline BLMT & -31.95 & 115.93 & $\begin{array}{l}2014.0 \\
2019.7 \\
\end{array}$ & $-0.76 \pm 0.5$ & $-0.23 \pm 1.0$ & $-0.68 \pm 0.72$ & DARR & -12.84 & 131.13 & $\begin{array}{l}2002.5 \\
2008.1 \\
\end{array}$ & $1.81 \pm 1.52$ & $-0.02 \pm 1.0$ & $1.92 \pm 0.72$ \\
\hline BNDY & -24.91 & 152.32 & $\begin{array}{l}2007.7 \\
2019.7 \\
\end{array}$ & $-0.66 \pm 0.39$ & $-0.12 \pm 1.0$ & $-0.78 \pm 0.47$ & DARW & -12.84 & 131.13 & $\begin{array}{l}1994.7 \\
2019.7 \\
\end{array}$ & $-0.75 \pm 0.41$ & $-0.02 \pm 1.0$ & $-0.7 \pm 0.48$ \\
\hline BNLA & -36.54 & 146.01 & $\begin{array}{l}2014.0 \\
2019.6 \\
\end{array}$ & $-1.49 \pm 1.38$ & $-0.0 \pm 1.0$ & $-1.54 \pm 0.67$ & DIXL & -23.94 & 150.27 & $\begin{array}{l}2013.3 \\
2017.8 \\
\end{array}$ & $-2.2 \pm 0.81$ & $-0.01 \pm 1.0$ & $-2.39 \pm 0.59$ \\
\hline BOLC & -37.71 & 142.84 & $\begin{array}{l}2013.4 \\
2019.6 \\
\end{array}$ & $-1.5 \pm 0.96$ & $-0.15 \pm 1.0$ & $-1.27 \pm 0.59$ & DODA & -13.83 & 131.19 & $\begin{array}{l}2009.8 \\
2019.7 \\
\end{array}$ & $0.33 \pm 0.46$ & $0.01 \pm 1.0$ & $0.43 \pm 0.5$ \\
\hline BRO1 & -18.0 & 122.21 & $\begin{array}{l}2010.5 \\
2019.7 \\
\end{array}$ & $-0.81 \pm 0.58$ & $-0.23 \pm 1.0$ & $-0.78 \pm 0.52$ & DPRT & -41.18 & 146.35 & $\begin{array}{l}2015.2 \\
2019.7 \\
\end{array}$ & $-2.11 \pm 0.95$ & $-0.18 \pm 1.0$ & $-2.11 \pm 0.82$ \\
\hline BRTN & -42.74 & 147.24 & $\begin{array}{l}2010.8 \\
2017.2 \\
\end{array}$ & $-0.03 \pm 1.03$ & $-0.16 \pm 1.0$ & $0.11 \pm 0.65$ & DWNI & -12.44 & 130.96 & $\begin{array}{l}2010.4 \\
2019.7 \\
\end{array}$ & $0.96 \pm 0.74$ & $-0.06 \pm 1.0$ & $1.06 \pm 0.54$ \\
\hline BUR1 & -41.05 & 145.91 & $\begin{array}{l}1999.3 \\
2007.4\end{array}$ & $-0.7 \pm 1.01$ & $-0.21 \pm 1.0$ & $-0.89 \pm 0.56$ & DYST & -22.6 & 148.5 & $\begin{array}{l}2013.3 \\
2018.3 \\
\end{array}$ & $0.96 \pm 1.51$ & $-0.0 \pm 1.0$ & $1.3 \pm 0.59$ \\
\hline BUR2 & -41.05 & 145.91 & $\begin{array}{l}2008.6 \\
2019.7\end{array}$ & $-0.68 \pm 0.49$ & $-0.21 \pm 1.0$ & $-0.67 \pm 0.47$ & EDS1 & -25.38 & 151.12 & $\begin{array}{l}2014.6 \\
2019.6\end{array}$ & $-1.22 \pm 0.73$ & $0.01 \pm 1.0$ & $-1.12 \pm 0.78$ \\
\hline BURA & -30.53 & 117.17 & $\begin{array}{l}2008.9 \\
2019.6\end{array}$ & $-0.21 \pm 0.51$ & $-0.12 \pm 1.0$ & $-0.2 \pm 0.50$ & ENSH & -23.48 & 148.52 & $\begin{array}{l}2010.7 \\
2019.7\end{array}$ & $-0.53 \pm 0.78$ & $-0.0 \pm 1.0$ & $-0.38 \pm 0.53$ \\
\hline BUSS & -33.65 & 115.35 & $\begin{array}{l}2014.9 \\
2019.7 \\
\end{array}$ & $-1.27 \pm 0.67$ & $-0.35 \pm 1.0$ & $-1.19 \pm 0.73$ & ESPA & -33.87 & 121.89 & $\begin{array}{l}2008.5 \\
2019.7 \\
\end{array}$ & $-0.26 \pm 0.41$ & $-0.34 \pm 1.0$ & $-0.42 \pm 0.47$ \\
\hline CAN3 & -20.29 & 148.67 & $\begin{array}{l}2013.0 \\
2019.7 \\
\end{array}$ & $-0.48 \pm 0.89$ & $-0.12 \pm 1.0$ & $-0.32 \pm 0.74$ & EXMT & -21.96 & 114.11 & $\begin{array}{l}2012.7 \\
2019.7 \\
\end{array}$ & $-0.11 \pm 0.87$ & $-0.32 \pm 1.0$ & $-0.12 \pm 0.58$ \\
\hline CBLT & -27.08 & 152.95 & $\begin{array}{l}2007.1 \\
2019.7 \\
\end{array}$ & $-0.83 \pm 0.61$ & $-0.11 \pm 1.0$ & $-0.91 \pm 0.49$ & FLND & -40.21 & 148.24 & $\begin{array}{l}2012.9 \\
2019.7 \\
\end{array}$ & $-1.04 \pm 1.2$ & $-0.31 \pm 1.0$ & $-1.14 \pm 0.68$ \\
\hline CBRK & -33.35 & 138.21 & $\begin{array}{l}2010.7 \\
2017.3 \\
\end{array}$ & $-0.54 \pm 1.06$ & $-0.14 \pm 1.0$ & $-0.68 \pm 0.64$ & FROY & -18.13 & 125.8 & $\begin{array}{l}2012.7 \\
2019.7 \\
\end{array}$ & $0.69 \pm 0.78$ & $-0.03 \pm 1.0$ & $0.83 \pm 0.59$ \\
\hline
\end{tabular}


1615 Table S5. Continued.

\begin{tabular}{|c|c|c|c|c|c|c|c|c|c|c|c|c|c|}
\hline $\begin{array}{c}\text { GPS } \\
\text { name }\end{array}$ & $\begin{array}{l}\text { Lat } \\
\text { (de) }\end{array}$ & $\begin{array}{l}\text { Lon } \\
\text { (deg) }\end{array}$ & $\begin{array}{l}\text { Time } \\
\text { Span }\end{array}$ & $\begin{array}{c}\text { Hector } \\
\text { VLM } \\
(\mathbf{m m} / \mathbf{y r})\end{array}$ & $\begin{array}{c}\text { GIA VLM } \\
(\mathbf{m m} / \mathbf{y r})\end{array}$ & $\underset{(\mathrm{mm} / \mathbf{y r})}{\operatorname{Our} \text { VLM }}$ & $\begin{array}{c}\text { GPS } \\
\text { name }\end{array}$ & $\begin{array}{c}\text { Lat } \\
\text { (deg) }\end{array}$ & $\begin{array}{l}\text { Lon } \\
\text { (deg) }\end{array}$ & $\begin{array}{l}\text { Time } \\
\text { span }\end{array}$ & $\begin{array}{c}\text { Hector } \\
\text { VLM } \\
(\mathbf{m m} / \mathbf{y r})\end{array}$ & $\begin{array}{c}\text { GIA VLM } \\
\text { (mm/yr) }\end{array}$ & $\begin{array}{c}\text { Our VLM } \\
(\mathbf{m m} / \mathbf{y r})\end{array}$ \\
\hline FTDN & -33.86 & 151.23 & $\begin{array}{l}2012.5 \\
2019.7\end{array}$ & $-0.53 \pm 0.74$ & $-0.2 \pm 1.0$ & $-0.61 \pm 0.59$ & LAUN & -41.43 & 147.15 & $\begin{array}{l}2010.7 \\
2019.7 \\
\end{array}$ & $-0.4 \pm 0.63$ & $-0.16 \pm 1.0$ & $-0.27 \pm 0.52$ \\
\hline GASC & -24.63 & 115.34 & \begin{tabular}{|l|}
2013.8 \\
2019.7 \\
\end{tabular} & $-0.61 \pm 0.92$ & $-0.07 \pm 1.0$ & $-0.55 \pm 0.74$ & LDHI & -31.54 & 159.08 & $\begin{array}{l}2010.0 \\
2019.2 \\
\end{array}$ & $-1.08 \pm 0.56$ & $-0.37 \pm 1.0$ & $-1.16 \pm 0.53$ \\
\hline GATT & -27.54 & 152.33 & $\begin{array}{l}2008.2 \\
2019.5 \\
\end{array}$ & $-0.21 \pm 1.04$ & $-0.03 \pm 1.0$ & $-0.16 \pm 0.53$ & LEAR & -22.22 & 114.1 & $\begin{array}{l}2011.7 \\
2019.7 \\
\end{array}$ & $0.26 \pm 0.93$ & $-0.29 \pm 1.0$ & $0.32 \pm 0.53$ \\
\hline GERO & -28.78 & 114.61 & $\begin{array}{l}2015.2 \\
2019.7\end{array}$ & $-2.05 \pm 0.78$ & $-0.33 \pm 1.0$ & $-1.98 \pm 0.83$ & LIAW & -41.9 & 146.67 & $\begin{array}{l}2008.8 \\
2019.7\end{array}$ & $-0.69 \pm 0.49$ & $-0.12 \pm 1.0$ & $-0.66 \pm 0.46$ \\
\hline GLAD & -23.84 & 151.25 & $\begin{array}{l}2010.7 \\
2019.7 \\
\end{array}$ & $-0.22 \pm 0.85$ & $-0.08 \pm 1.0$ & $-0.11 \pm 0.55$ & LILY & -41.25 & 147.21 & $\begin{array}{l}2015.2 \\
2019.7 \\
\end{array}$ & $-0.48 \pm 1.46$ & $-0.18 \pm 1.0$ & $-0.36 \pm 0.88$ \\
\hline GLNC & -42.83 & 147.27 & $\begin{array}{l}2015.6 \\
2019.7\end{array}$ & $-2.65 \pm 1.28$ & $-0.17 \pm 1.0$ & $-2.6 \pm 1.2$ & LKYA & -12.46 & 130.82 & $\begin{array}{l}2011.6 \\
2019.7\end{array}$ & $0.57 \pm 0.4$ & $-0.07 \pm 1.0$ & $0.46 \pm 0.53$ \\
\hline GOOM & -31.4 & 116.85 & $\begin{array}{l}2014.2 \\
2019.6\end{array}$ & $0.29 \pm 0.77$ & $-0.13 \pm 1.0$ & $0.24 \pm 0.72$ & LORD & -31.52 & 159.06 & $\begin{array}{l}2009.5 \\
2019.7\end{array}$ & $-1.27 \pm 0.52$ & $-0.37 \pm 1.0$ & $-1.3 \pm 0.51$ \\
\hline GRN1 & -33.86 & 116.06 & $\begin{array}{l}2015.0 \\
2019.7\end{array}$ & $-1.37 \pm 0.76$ & $-0.26 \pm 1.0$ & $-1.3 \pm 0.83$ & LUC2 & -37.04 & 140.27 & $\begin{array}{l}2013.6 \\
2017.1\end{array}$ & $-0.47 \pm 1.03$ & $-0.25 \pm 1.0$ & $-0.45 \pm 1.18$ \\
\hline GYM2 & -26.19 & 152.66 & $\begin{array}{l}2011.0 \\
2018.0\end{array}$ & $-0.89 \pm 1.08$ & $-0.1 \pm 1.0$ & $-0.8 \pm 0.59$ & MAIN & -14.05 & 134.09 & $\begin{array}{l}2012.2 \\
2019.7\end{array}$ & $2.04 \pm 0.66$ & $0.05 \pm 1.0$ & $2.14 \pm 0.57$ \\
\hline HBAY & -25.28 & 152.83 & $\begin{array}{l}2010.7 \\
2019.7\end{array}$ & $-0.22 \pm 0.94$ & $-0.16 \pm 1.0$ & $-0.13 \pm 0.54$ & MAIT & -34.37 & 137.67 & $\begin{array}{l}2010.7 \\
2017.8\end{array}$ & $-0.18 \pm 0.94$ & $-0.24 \pm 1.0$ & $-0.27 \pm 0.62$ \\
\hline HBG2 & -34.14 & 150.94 & $\begin{array}{l}2013.9 \\
2019.7 \\
\end{array}$ & $-0.32 \pm 1.02$ & $-0.18 \pm 1.0$ & $-0.28 \pm 0.67$ & MANY & -35.05 & 141.06 & $\begin{array}{l}2013.4 \\
2018.9 \\
\end{array}$ & $-2.16 \pm 0.99$ & $-0.07 \pm 1.0$ & $-2.08 \pm 0.68$ \\
\hline HIL1 & -31.83 & 115.74 & $\begin{array}{l}1997.7 \\
2019.7 \\
\end{array}$ & $-2.28 \pm 0.32$ & $-0.26 \pm 1.0$ & $-2.17 \pm 0.55$ & MARY & -37.01 & 143.76 & $\begin{array}{l}2013.4 \\
2019.6 \\
\end{array}$ & $-0.97 \pm 0.72$ & $-0.05 \pm 1.0$ & $-0.83 \pm 0.59$ \\
\hline HNIS & -10.59 & 142.3 & $\begin{array}{l}2010.7 \\
2019.5 \\
\end{array}$ & $1.72 \pm 1.04$ & $-0.05 \pm 1.0$ & $1.84 \pm 0.57$ & MBH4 & -25.53 & 152.71 & $\begin{array}{l}2012.8 \\
2018.9 \\
\end{array}$ & $-1.27 \pm 1.01$ & $-0.13 \pm 1.0$ & $-1.14 \pm 0.59$ \\
\hline HNSB & -33.70 & 151.1 & $\begin{array}{l}2012.8 \\
2019.7 \\
\end{array}$ & $-0.83 \pm 1.62$ & $-0.17 \pm 1.0$ & $-0.71 \pm 0.57$ & $\mathrm{MCKN}$ & -35.52 & 138.65 & $\begin{array}{l}2014.1 \\
2018.6 \\
\end{array}$ & $-0.89 \pm 0.88$ & $-0.28 \pm 1.0$ & $-0.98 \pm 0.79$ \\
\hline HOB2 & -42.80 & 147.44 & $\begin{array}{l}1994.5 \\
2019.7 \\
\end{array}$ & $-0.97 \pm 0.30$ & $-0.18 \pm 1.0$ & $-1.04 \pm 0.48$ & MCLV & -35.22 & 138.54 & $\begin{array}{l}2010.7 \\
2015.3 \\
\end{array}$ & $2.38 \pm 0.81$ & $-0.25 \pm 1.0$ & $2.53 \pm 0.96$ \\
\hline HRSM & -36.72 & 142.17 & $\begin{array}{l}2013.4 \\
2019.6 \\
\end{array}$ & $-1.14 \pm 0.73$ & $-0.07 \pm 1.0$ & $-1.05 \pm 0.61$ & MEDO & -26.76 & 114.61 & $\begin{array}{l}2013.5 \\
2019.7\end{array}$ & $0.39 \pm 1.1$ & $-0.2 \pm 1.0$ & $0.55 \pm 0.64$ \\
\hline INSF & -17.53 & 146.03 & $\begin{array}{l}2013.7 \\
2018.8 \\
\end{array}$ & $-0.05 \pm 1.25$ & $-0.12 \pm 1.0$ & $0.07 \pm 0.84$ & MIDG & -20.64 & 148.71 & $\begin{array}{l}2011.7 \\
2019.7 \\
\end{array}$ & $0.43 \pm 0.83$ & $-0.08 \pm 1.0$ & $0.56 \pm 0.57$ \\
\hline IPS2 & -27.61 & 152.76 & $\begin{array}{l}2010.0 \\
2014.2\end{array}$ & $-2.49 \pm 1.21$ & $-0.07 \pm 1.0$ & $-2.62 \pm 1.05$ & MNDH & -32.53 & 115.71 & $\begin{array}{l}2013.7 \\
2017.8\end{array}$ & $-0.62 \pm 0.9$ & $-0.22 \pm 1.0$ & $-0.49 \pm 1.01$ \\
\hline IPSR & -27.61 & 152.76 & $\begin{array}{l}2014.7 \\
2019.7\end{array}$ & $-0.69 \pm 0.70$ & $-0.07 \pm 1.0$ & $-0.62 \pm 0.73$ & MNGO & -38.78 & 143.65 & $\begin{array}{l}2012.1 \\
2019.7\end{array}$ & $-0.57 \pm 1.24$ & $-0.3 \pm 1.0$ & $-0.67 \pm 0.59$ \\
\hline JAB2 & -12.66 & 132.89 & $\begin{array}{l}2008.7 \\
2019.7 \\
\end{array}$ & $0.81 \pm 0.55$ & $0.05 \pm 1.0$ & $0.67 \pm 0.5$ & MNTO & -24.87 & 151.13 & $\begin{array}{l}2014.3 \\
2019.7 \\
\end{array}$ & $1.02 \pm 1.02$ & $-0.01 \pm 1.0$ & $1.22 \pm 0.72$ \\
\hline JOON & -31.73 & 115.75 & $\begin{array}{l}2013.6 \\
2019.7 \\
\end{array}$ & $0.48 \pm 0.83$ & $-0.27 \pm 1.0$ & $0.42 \pm 0.63$ & MOBS & -37.83 & 144.98 & $\begin{array}{l}2002.8 \\
2019.7 \\
\end{array}$ & $-0.91 \pm 0.38$ & $-0.12 \pm 1.0$ & $-0.87 \pm 0.34$ \\
\hline KARO & -35.10 & 139.89 & $\begin{array}{l}2010.7 \\
2017.3 \\
\end{array}$ & $-0.39 \pm 1.03$ & $-0.13 \pm 1.0$ & $-0.49 \pm 0.62$ & MOOR & -37.40 & 142.13 & $\begin{array}{l}2013.4 \\
2019.6 \\
\end{array}$ & $-1.3 \pm 0.99$ & $-0.14 \pm 1.0$ & $-1.17 \pm 0.65$ \\
\hline KARR & -20.98 & 117.1, & $\begin{array}{l}1994.8 \\
2019.7 \\
\end{array}$ & $-0.74 \pm 0.32$ & $-0.19 \pm 1.0$ & $-0.71 \pm 0.48$ & MRNO & -37.72 & 141.55 & $\begin{array}{l}2013.4 \\
2019.6 \\
\end{array}$ & $-0.51 \pm 0.84$ & $-0.22 \pm 1.0$ & $-0.3 \pm 0.62$ \\
\hline KAT1 & -14.38 & 132.15 & $\begin{array}{l}2010.2 \\
2019.7 \\
\end{array}$ & $0.45 \pm 0.58$ & $0.04 \pm 1.0$ & $0.51 \pm 0.51$ & MRNT & -38.23 & 145.07 & $\begin{array}{l}2014.0 \\
2019.6 \\
\end{array}$ & $-1.43 \pm 1.42$ & $-0.17 \pm 1.0$ & $-1.34 \pm 0.67$ \\
\hline KAT2 & -14.38 & 132.15 & $\begin{array}{l}2010.2 \\
2019.7 \\
\end{array}$ & $-0.08 \pm 0.42$ & $0.04 \pm 1.0$ & $0.03 \pm 0.52$ & MRO1 & -26.7 & 116.64 & $\begin{array}{l}2013.8 \\
2019.7 \\
\end{array}$ & $-0.19 \pm 0.74$ & $-0.1 \pm 1.0$ & $-0.06 \pm 0.64$ \\
\hline KDNA & -33.97 & 137.72 & $\begin{array}{l}2012.1 \\
2015.9 \\
\end{array}$ & $0.34 \pm 0.81$ & $-0.2 \pm 1.0$ & $0.34 \pm 1.35$ & MRT1 & -19.76 & 146.83 & $\begin{array}{l}2014.9 \\
2019.6 \\
\end{array}$ & $-1.82 \pm 1.01$ & $-0.06 \pm 1.0$ & $-1.90 \pm 0.87$ \\
\hline KELN & -31.62 & 117.7 & \begin{tabular}{|l}
2009.1 \\
2019.6 \\
\end{tabular} & $-0.97 \pm 0.41$ & $-0.08 \pm 1.0$ & $-0.96 \pm 0.47$ & MRT2 & -19.46 & 147.48 & $\begin{array}{l}2014.9 \\
2019.6 \\
\end{array}$ & $-1.8 \pm 0.61$ & $-0.13 \pm 1.0$ & $-1.69 \pm 0.78$ \\
\hline KGIS & -39.94 & 143.85 & $\begin{array}{l}2013.9 \\
2019.7\end{array}$ & $-1.67 \pm 1.10$ & $-0.41 \pm 1.0$ & $-1.72 \pm 0.66$ & MRT3 & -19.33 & 146.52 & $\begin{array}{l}2014.9 \\
2019.6\end{array}$ & $0.95 \pm 1.06$ & $-0.07 \pm 1.0$ & $+1.00 \pm 1.00$ \\
\hline KILK & -26.08 & 152.25 & $\begin{array}{l}2013.5 \\
2019.7\end{array}$ & $-0.16 \pm 0.73$ & $-0.05 \pm 1.0$ & $-0.15 \pm 0.64$ & MRYB & -35.15 & 139.26 & $\begin{array}{l}2010.7 \\
2019.6 \\
\end{array}$ & $0.69 \pm 0.87$ & $-0.18 \pm 1.0$ & $0.87 \pm 0.51$ \\
\hline KIN2 & -26.54 & 151.84 & $\begin{array}{l}2013.6 \\
2019.7 \\
\end{array}$ & $0.30 \pm 0.79$ & $-0.0 \pm 1.0$ & $0.16 \pm 0.66$ & MTB2 & -35.06 & 138.86 & $\begin{array}{l}2015.0 \\
2019.7 \\
\end{array}$ & $-0.27 \pm 0.69$ & $-0.21 \pm 1.0$ & $-0.16 \pm 0.76$ \\
\hline KJNG & -33.51 & 150.79 & $\begin{array}{l}2012.8 \\
2019.7 \\
\end{array}$ & $0.93 \pm 0.90$ & $-0.11 \pm 1.0$ & $0.85 \pm 0.63$ & MTEM & -37.59 & 143.45 & $\begin{array}{l}2011.4 \\
2019.7 \\
\end{array}$ & $0.25 \pm 0.8$ & $-0.11 \pm 1.0$ & $0.39 \pm 0.53$ \\
\hline KOUM & -21.61 & 149.24 & $\begin{array}{l}2011.7 \\
2019.7 \\
\end{array}$ & $-1.03 \pm 0.96$ & $-0.05 \pm 1.0$ & $-0.91 \pm 0.59$ & MTGA & -37.83 & 140.78 & $\begin{array}{l}2010.9 \\
2019.7 \\
\end{array}$ & $-0.91 \pm 0.58$ & $-0.31 \pm 1.0$ & $-0.82 \pm 0.5$ \\
\hline КTHA & -20.73 & 116.84 & $\begin{array}{l}2014.1 \\
2019.7 \\
\end{array}$ & $0.21 \pm 0.92$ & $-0.24 \pm 1.0$ & $0.17 \pm 0.67$ & MULG & -30.28 & 134.06 & $\begin{array}{l}2014.7 \\
2019.7 \\
\end{array}$ & $-0.76 \pm 1.16$ & $-0.11 \pm 1.0$ & $-0.69 \pm 0.78$ \\
\hline KURR & -32.80 & 151.49 & $\begin{array}{l}2010.7 \\
2019.5 \\
\end{array}$ & $-0.34 \pm 0.98$ & $-0.14 \pm 1.0$ & $-0.32 \pm 0.59$ & MURM & -35.06 & 149.09 & $\begin{array}{l}2010.9 \\
2019.6 \\
\end{array}$ & $-1.01 \pm 0.7$ & $-0.04 \pm 1.0$ & $-0.98 \pm 0.53$ \\
\hline LAM1 & -35.33 & 140.51 & $\begin{array}{l}2010.7 \\
2017.4 \\
\end{array}$ & $-1.13 \pm 0.94$ & $-0.1 \pm 1.0$ & $-1.25 \pm 0.63$ & MYAP & -33.06 & 115.74 & $\begin{array}{l}2015.8 \\
2019.7 \\
\end{array}$ & $-0.72 \pm 1.51$ & $-0.18 \pm 1.0$ & $-0.59 \pm 1.16$ \\
\hline LARR & -15.57 & 133.21 & $\begin{array}{l}2009.8 \\
2019.7 \\
\end{array}$ & $-0.4 \pm 0.45$ & $0.01 \pm 1.0$ & $-0.51 \pm 0.49$ & NELN & -38.05 & 141.01 & $\begin{array}{l}2013.4 \\
2019.6 \\
\end{array}$ & $0.09 \pm 0.64$ & $-0.32 \pm 1.0$ & $-0.0 \pm 0.59$ \\
\hline
\end{tabular}

1616 
1617 Table S5. Continued.

\begin{tabular}{|c|c|c|c|c|c|c|c|c|c|c|c|c|c|}
\hline $\begin{array}{c}\text { GPS } \\
\text { name }\end{array}$ & $\begin{array}{l}\text { Lat } \\
\text { (de) }\end{array}$ & $\begin{array}{l}\text { Lon } \\
\text { (deg) }\end{array}$ & $\begin{array}{l}\text { Time } \\
\text { Span }\end{array}$ & $\begin{array}{c}\text { Hector } \\
\text { VLM } \\
(\mathbf{m m} / \mathbf{y r})\end{array}$ & $\begin{array}{c}\text { GIA VLM } \\
(\mathbf{m m} / \mathbf{y r})\end{array}$ & $\begin{array}{c}\text { Our VLM } \\
(\mathbf{m m} / \mathbf{y r})\end{array}$ & $\begin{array}{c}\text { GPS } \\
\text { name }\end{array}$ & $\begin{array}{c}\text { Lat } \\
\text { (deg) }\end{array}$ & $\begin{array}{l}\text { Lon } \\
\text { (deg) }\end{array}$ & $\begin{array}{l}\text { Time } \\
\text { span }\end{array}$ & $\begin{array}{c}\text { Hector } \\
\text { VLM } \\
(\mathbf{m m} / \mathbf{y r})\end{array}$ & $\begin{array}{c}\text { GIA VLM } \\
(\mathbf{m m} / \mathbf{y r})\end{array}$ & $\underset{(\mathbf{m m} / \mathbf{y r})}{\text { Our VLM }}$ \\
\hline NEWE & -32.92 & 151.79 & $\begin{array}{l}2012.5 \\
2019.7\end{array}$ & $0.08 \pm 0.68$ & $-0.19 \pm 1.0$ & $+0.14 \pm 0.6$ & SYDN & -33.78 & 151.15 & $\begin{array}{l}2004.4 \\
2019.7 \\
\end{array}$ & $-0.45 \pm 0.65$ & $-0.18 \pm 1.0$ & $-0.45 \pm 0.39$ \\
\hline NHAV & -34.79 & 138.49 & $\begin{array}{l}2012.1 \\
2019.7 \\
\end{array}$ & $1.6 \pm 0.81$ & $-0.22 \pm 1.0$ & $+1.71 \pm 0.54$ & SYM1 & -35.34 & 149.16 & $\begin{array}{l}2014.7 \\
2019.7 \\
\end{array}$ & $-0.77 \pm 0.8$ & $-0.05 \pm 1.0$ & $-0.52 \pm 0.71$ \\
\hline NHIL & -36.31 & 141.65 & $\begin{array}{l}2010.6 \\
2019.7 \\
\end{array}$ & $1.48 \pm 0.88$ & $-0.07 \pm 1.0$ & $+1.4 \pm 0.57$ & TER4 & -23.96 & 148.78 & $\begin{array}{l}2013.3 \\
2019.7 \\
\end{array}$ & $-0.2 \pm 0.58$ & $-0.0 \pm 1.0$ & $0.04 \pm 0.59$ \\
\hline NNOR & -31.05 & 116.19 & $\begin{array}{l}2002.5 \\
2019.7\end{array}$ & $-1.21 \pm 0.65$ & $-0.19 \pm 1.0$ & $-1.14 \pm 0.33$ & THE1 & -24.95 & 150.08 & $\begin{array}{l}2013.4 \\
2017.4\end{array}$ & $-1.25 \pm 1.46$ & $0.01 \pm 1.0$ & $-1.0 \pm 1.34$ \\
\hline NORS & -32.26 & 121.79 & $\begin{array}{l}2009.2 \\
2019.7\end{array}$ & $-0.52 \pm 0.61$ & $-0.13 \pm 1.0$ & $-0.64 \pm 0.49$ & THEV & -32.13 & 133.7 & $\begin{array}{l}2013.2 \\
2019.7\end{array}$ & $0.7 \pm 1.52$ & $-0.27 \pm 1.0$ & $0.52 \pm 0.67$ \\
\hline ORA2 & -34.0 & 150.74 & \begin{tabular}{|l}
2012.8 \\
2018.9 \\
\end{tabular} & $-1.57 \pm 1.13$ & $-0.14 \pm 1.0$ & $-1.6 \pm 0.69$ & TID1 & -35.4 & 148.98 & $\begin{array}{l}1996.5 \\
2019.7 \\
\end{array}$ & $-0.54 \pm 0.65$ & $-0.04 \pm 1.0$ & $-0.34 \pm 0.48$ \\
\hline PERT & -31.8 & 115.89 & $\begin{array}{l}1994.2 \\
2019.7 \\
\end{array}$ & $-2.81 \pm 0.65$ & $-0.24 \pm 1.0$ & $-3.02 \pm 0.48$ & TID2 & -35.4 & 148.98 & $\begin{array}{l}1994.7 \\
2010.5 \\
\end{array}$ & $-0.13 \pm 0.65$ & $-0.04 \pm 1.0$ & $-0.08 \pm 0.31$ \\
\hline PINN & -35.26 & 140.91 & $\begin{array}{l}2012.1 \\
2017.7\end{array}$ & $0.99 \pm 1.3$ & $-0.08 \pm 1.0$ & $0.93 \pm 0.88$ & TIDB & -35.4 & 148.98 & $\begin{array}{l}1994.6 \\
2019.6\end{array}$ & $-0.8 \pm 0.65$ & $-0.04 \pm 1.0$ & $-0.65 \pm 0.48$ \\
\hline PNRY & -34.31 & 138.42 & $\begin{array}{l}2012.1 \\
2016.1\end{array}$ & $0.73 \pm 1.69$ & $-0.18 \pm 1.0$ & $0.82 \pm 1.23$ & TITG & -10.59 & 142.22 & $\begin{array}{l}2015.8 \\
2019.7\end{array}$ & $-1.66 \pm 0.81$ & $-0.05 \pm 1.0$ & $-1.44 \pm 1.29$ \\
\hline POCA & -38.62 & 143.0 & $\begin{array}{l}2013.4 \\
2019.6\end{array}$ & $-0.42 \pm 0.77$ & $-0.3 \pm 1.0$ & $-0.26 \pm 0.6$ & TNDA & -34.51 & 138.98 & $\begin{array}{l}2010.7 \\
2016.4\end{array}$ & $0.48 \pm 1.2$ & $-0.16 \pm 1.0$ & $0.67 \pm 0.74$ \\
\hline PRO1 & -26.16 & 151.6 & $\begin{array}{l}2011.1 \\
2019.7\end{array}$ & $-1.6 \pm 0.64$ & $0.01 \pm 1.0$ & $-1.65 \pm 0.54$ & TNGL & -24.49 & 150.57 & $\begin{array}{l}2013.4 \\
2019.7 \\
\end{array}$ & $-1.01 \pm 0.78$ & $-0.0 \pm 1.0$ & $-0.86 \pm 0.64$ \\
\hline PRTF & -38.38 & 142.24 & $\begin{array}{l}2013.4 \\
2019.6\end{array}$ & $0.12 \pm 0.76$ & $-0.29 \pm 1.0$ & $0.25 \pm 0.59$ & TOMP & -22.85 & 117.4 & $\begin{array}{l}2013.0 \\
2019.7\end{array}$ & $1.38 \pm 0.87$ & $-0.04 \pm 1.0$ & $1.57 \pm 0.67$ \\
\hline PTKL & -34.48 & 150.91 & $\begin{array}{l}2009.7 \\
2019.6 \\
\end{array}$ & $-0.53 \pm 0.62$ & $-0.2 \pm 1.0$ & $-0.39 \pm 0.51$ & TOOG & -27.08 & 152.37 & $\begin{array}{l}2014.5 \\
2019.7 \\
\end{array}$ & $0.07 \pm 0.59$ & $-0.04 \pm 1.0$ & $0.23 \pm 0.72$ \\
\hline PTLD & -38.34 & 141.61 & $\begin{array}{l}2009.7 \\
2019.7 \\
\end{array}$ & $-0.66 \pm 0.67$ & $-0.33 \pm 1.0$ & $-0.61 \pm 0.51$ & TOOW & -27.53 & 151.93 & $\begin{array}{l}2009.8 \\
2019.7\end{array}$ & $-0.56 \pm 0.87$ & $0.01 \pm 1.0$ & $-0.55 \pm 0.52$ \\
\hline PTSV & -35.09 & 138.49 & $\begin{array}{l}2010.7 \\
2019.7\end{array}$ & $-0.81 \pm 0.59$ & $-0.24 \pm 1.0$ & $-0.67 \pm 0.5$ & TOW2 & -19.27 & 147.06 & $\begin{array}{l}1995.0 \\
2019.7\end{array}$ & $-0.8 \pm 0.37$ & $-0.11 \pm 1.0$ & $-0.85 \pm 0.5$ \\
\hline QCLF & -38.27 & 144.64 & $\begin{array}{l}2013.4 \\
2019.6 \\
\end{array}$ & $-1.76 \pm 1.1$ & $-0.19 \pm 1.0$ & $-1.9 \pm 0.63$ & TRN1 & -26.8 & 151.9 & $\begin{array}{l}2011.6 \\
2019.6\end{array}$ & $0.64 \pm 1.4$ & $-0.0 \pm 1.0$ & $0.86 \pm 0.68$ \\
\hline RAVN & -33.6 & 120.07 & $\begin{array}{l}2010.3 \\
2019.6\end{array}$ & $-0.36 \pm 0.54$ & $-0.21 \pm 1.0$ & $-0.30 \pm 0.49$ & TURO & -36.04 & 150.12 & $\begin{array}{l}2011.3 \\
2019.7\end{array}$ & $-0.16 \pm 0.62$ & $-0.2 \pm 1.0$ & $-0.12 \pm 0.55$ \\
\hline RHPT & -41.07 & 145.96 & $\begin{array}{l}2008.0 \\
2019.7\end{array}$ & $-1.8 \pm 0.4$ & $-0.21 \pm 1.0$ & $-1.93 \pm 0.47$ & TWED & -28.35 & 153.40 & $\begin{array}{l}2010.7 \\
2019.0\end{array}$ & $-2.59 \pm 1.26$ & $-0.16 \pm 1.0$ & $-2.67 \pm 0.6$ \\
\hline RID1 & -23.29 & 150.21 & $\begin{array}{l}2013.0 \\
2019.7\end{array}$ & $-2.8 \pm 0.98$ & $-0.03 \pm 1.0$ & $-2.65 \pm 0.62$ & UNX2 & -33.92 & 151.23 & $\begin{array}{l}2013.4 \\
2017.6\end{array}$ & $-0.66 \pm 1.44$ & $-0.2 \pm 1.0$ & $-0.49 \pm 1.26$ \\
\hline ROBI & -28.08 & 153.38 & $\begin{array}{l}2007.1 \\
2019.7\end{array}$ & $-0.48 \pm 0.52$ & $-0.16 \pm 1.0$ & $-0.57 \pm 0.49$ & UNX3 & -33.92 & 151.23 & $\begin{array}{l}2013.4 \\
2017.6\end{array}$ & $-0.49 \pm 1.44$ & $-0.2 \pm 1.0$ & $-0.41 \pm 1.3$ \\
\hline ROC2 & -23.38 & 150.51 & \begin{tabular}{|l}
2014.0 \\
2018.3 \\
\end{tabular} & $-2.26 \pm 1.15$ & $-0.04 \pm 1.0$ & $-2.07 \pm 1.12$ & WAGN & -33.33 & 117.41 & $\begin{array}{l}2010.2 \\
2019.7 \\
\end{array}$ & $-1.24 \pm 0.58$ & $-0.12 \pm 1.0$ & $-1.09 \pm 0.49$ \\
\hline ROS5 & -24.63 & 151.91 & $\begin{array}{l}2014.3 \\
2019.7 \\
\end{array}$ & $-2.07 \pm 0.58$ & $-0.09 \pm 1.0$ & $-1.81 \pm 0.68$ & WAIK & -34.2 & 140.0 & $\begin{array}{l}2010.7 \\
2016.4 \\
\end{array}$ & $-0.31 \pm 1.14$ & $-0.09 \pm 1.0$ & $-0.44 \pm 0.71$ \\
\hline RSBY & -23.16 & 150.79 & $\begin{array}{l}2011.6 \\
2019.7 \\
\end{array}$ & $0.88 \pm 0.62$ & $-0.07 \pm 1.0$ & $0.75 \pm 0.63$ & WARW & -28.21 & 152.03 & $\begin{array}{l}2010.7 \\
2019.7 \\
\end{array}$ & $0.5 \pm 0.73$ & $0.0 \pm 1.0$ & $0.3 \pm 0.53$ \\
\hline SG36 & -37.91 & 145.13 & $\begin{array}{l}2003.9 \\
2010.8\end{array}$ & $-0.4 \pm 0.86$ & $-0.12 \pm 1.0$ & $-0.26 \pm 0.59$ & WEDD & -36.43 & 143.61 & $\begin{array}{l}2013.4 \\
2019.6\end{array}$ & $-0.15 \pm 0.88$ & $-0.03 \pm 1.0$ & $-0.16 \pm 0.6$ \\
\hline SKIP & -37.68 & 143.36 & $\begin{array}{l}2013.4 \\
2019.6 \\
\end{array}$ & $1.35 \pm 0.63$ & $-0.13 \pm 1.0$ & $1.54 \pm 0.6$ & WEND & -37.54 & 143.83 & $\begin{array}{l}2015.5 \\
2019.6\end{array}$ & $0.0 \pm 1.41$ & $-0.1 \pm 1.0$ & $0.24 \pm 0.99$ \\
\hline SPA7 & -32.01 & 115.9 & $\begin{array}{l}2014.2 \\
2019.7\end{array}$ & $-1.93 \pm 1.07$ & $-0.24 \pm 1.0$ & $-2.26 \pm 0.69$ & WIL3 & -33.03 & 116.88 & $\begin{array}{l}2010.7 \\
2016.5\end{array}$ & $-0.31 \pm 1.04$ & $-0.13 \pm 1.0$ & $-0.48 \pm 0.79$ \\
\hline SPBY & -42.55 & 147.93 & $\begin{array}{l}2008.8 \\
2019.7\end{array}$ & $-1.02 \pm 0.49$ & $-0.19 \pm 1.0$ & $-1.1 \pm 0.47$ & WLAL & -19.78 & 120.64 & $\begin{array}{l}2011.9 \\
2019.7\end{array}$ & $0.43 \pm 0.82$ & $-0.23 \pm 1.0$ & $0.63 \pm 0.53$ \\
\hline SSCK & -40.96 & 145.58 & $\begin{array}{l}2012.1 \\
2017.8\end{array}$ & $-1.72 \pm 1.15$ & $-0.24 \pm 1.0$ & $-1.55 \pm 0.7$ & WNBL & -38.38 & 142.48 & $\begin{array}{l}2013.9 \\
2019.6\end{array}$ & $-1.16 \pm 0.58$ & $-0.28 \pm 1.0$ & $-1.12 \pm 0.67$ \\
\hline STA2 & -36.62 & 143.26 & $\begin{array}{l}2013.4 \\
2017.3\end{array}$ & $-0.02 \pm 1.38$ & $-0.04 \pm 1.0$ & $0.06 \pm 1.01$ & WOOL & -27.48 & 153.04 & $\begin{array}{l}2007.1 \\
2018.4\end{array}$ & $-1.04 \pm 0.7$ & $-0.12 \pm 1.0$ & $-0.93 \pm 0.5$ \\
\hline STLW & -22.48 & 149.59 & $\begin{array}{l}2013.6 \\
2019.7 \\
\end{array}$ & $-0.52 \pm 1.17$ & $-0.03 \pm 1.0$ & $-0.3 \pm 0.7$ & WORI & -37.78 & 145.53 & $\begin{array}{l}2014.0 \\
2019.6 \\
\end{array}$ & $-2.08 \pm 0.81$ & $-0.1 \pm 1.0$ & $-1.85 \pm 0.71$ \\
\hline STNY & -38.38 & 145.21 & $\begin{array}{l}2011.4 \\
2019.7\end{array}$ & $-0.37 \pm 0.85$ & $-0.19 \pm 1.0$ & $-0.16 \pm 0.53$ & YAR1 & -29.05 & 115.35 & $\begin{array}{l}1994.0 \\
2002.4\end{array}$ & $-0.6 \pm 1.05$ & $-0.24 \pm 1.0$ & $-0.77 \pm 0.53$ \\
\hline STR1 & -35.32 & 149.01 & $\begin{array}{l}1998.5 \\
2019.7\end{array}$ & $-0.75 \pm 0.32$ & $-0.04 \pm 1.0$ & $-0.68 \pm 0.48$ & YAR2 & -29.05 & 115.35 & $\begin{array}{l}1996.5 \\
2019.6\end{array}$ & $0.41 \pm 0.34$ & $-0.24 \pm 1.0$ & $0.56 \pm 0.48$ \\
\hline STR2 & -35.32 & 149.01 & $\begin{array}{l}2002.5 \\
2019.7 \\
\end{array}$ & $-0.72 \pm 0.48$ & $-0.04 \pm 1.0$ & $-0.63 \pm 0.35$ & YAR3 & -29.05 & 115.35 & $\begin{array}{l}2007.5 \\
2019.6\end{array}$ & $-0.5 \pm 0.41$ & $-0.24 \pm 1.0$ & $-0.56 \pm 0.46$ \\
\hline STR4 & -35.32 & 149.01 & $\begin{array}{l}2010.7 \\
2019.7\end{array}$ & $-0.75 \pm 0.64$ & $-0.04 \pm 1.0$ & $-0.99 \pm 0.54$ & YNKI & -38.81 & 146.22 & $\begin{array}{l}2013.4 \\
2019.7 \\
\end{array}$ & $0.21 \pm 1.01$ & $-0.23 \pm 1.0$ & $0.19 \pm 0.62$ \\
\hline STRH & -37.73 & 141.14 & $\begin{array}{l}2013.4 \\
2019.6\end{array}$ & $-2.53 \pm 0.92$ & $-0.26 \pm 1.0$ & $-2.2 \pm 0.75$ & YOR5 & -35.02 & 137.61 & $\begin{array}{l}2014.3 \\
2018.9\end{array}$ & $2.07 \pm 0.81$ & $-0.31 \pm 1.0$ & $1.76 \pm 1.03$ \\
\hline
\end{tabular}




\subsection{Residuals and a posteriori analysis}

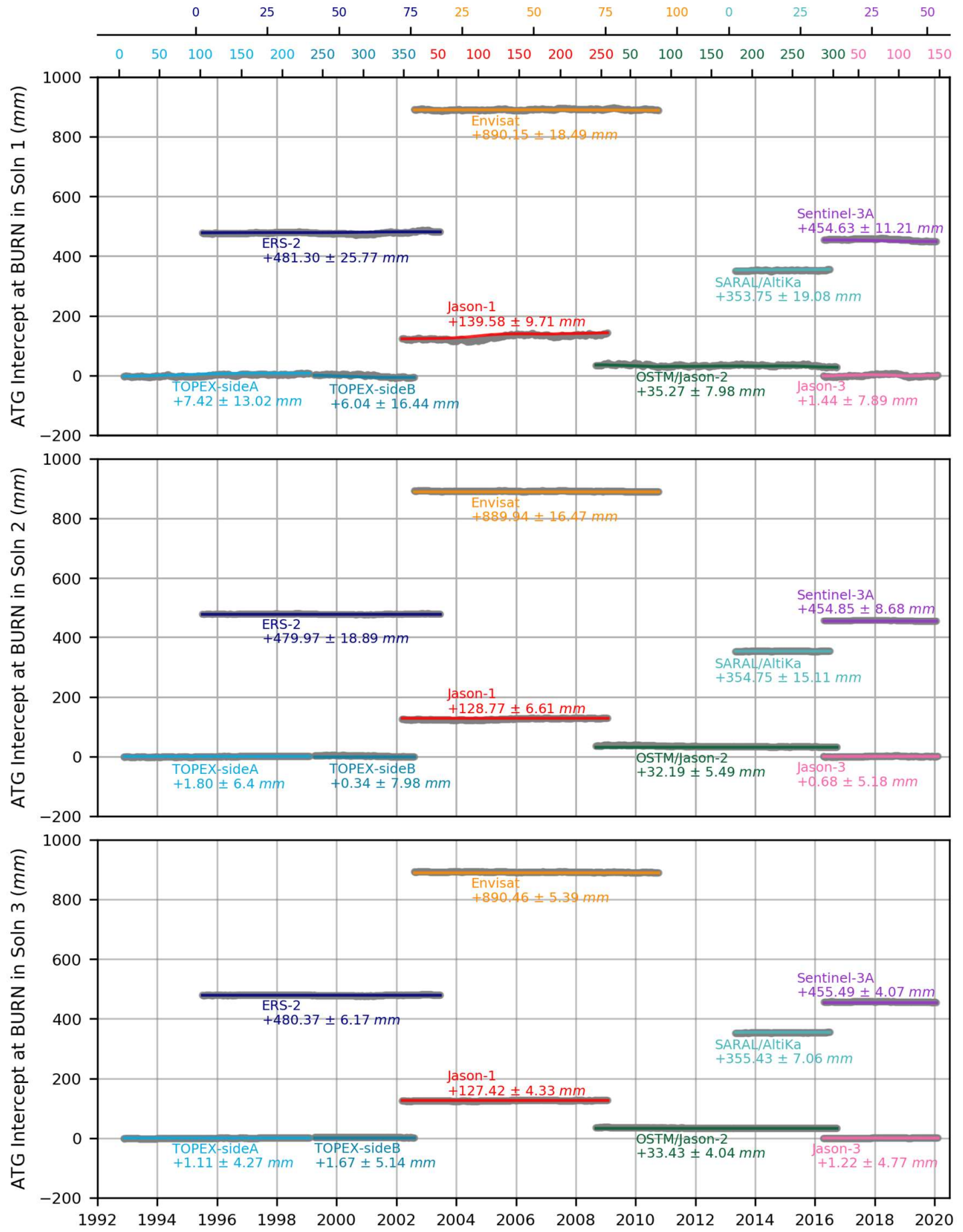

Figure S27. Cycle-by-cycle estimate of ATG intercepts for a representative CP in the vicinity of BURN TG in the final iterations of (top) Solution 1, (middle) Solution 2 and (bottom) Solution 3 within our multi-stage approach. The grey lines show the forward filtering estimates, while the colored lines show the return smoothing results. The estimated intercepts loosely varied in time in Solution 1 due to the unmodeled signals, while treated as time-fixed quantities in the subsequent Solutions 2 and 3. The averaged smoothing estimates are annotated for each solution. Note all intercepts are relative to the median estimate of TOPEX-side A in each solution. 

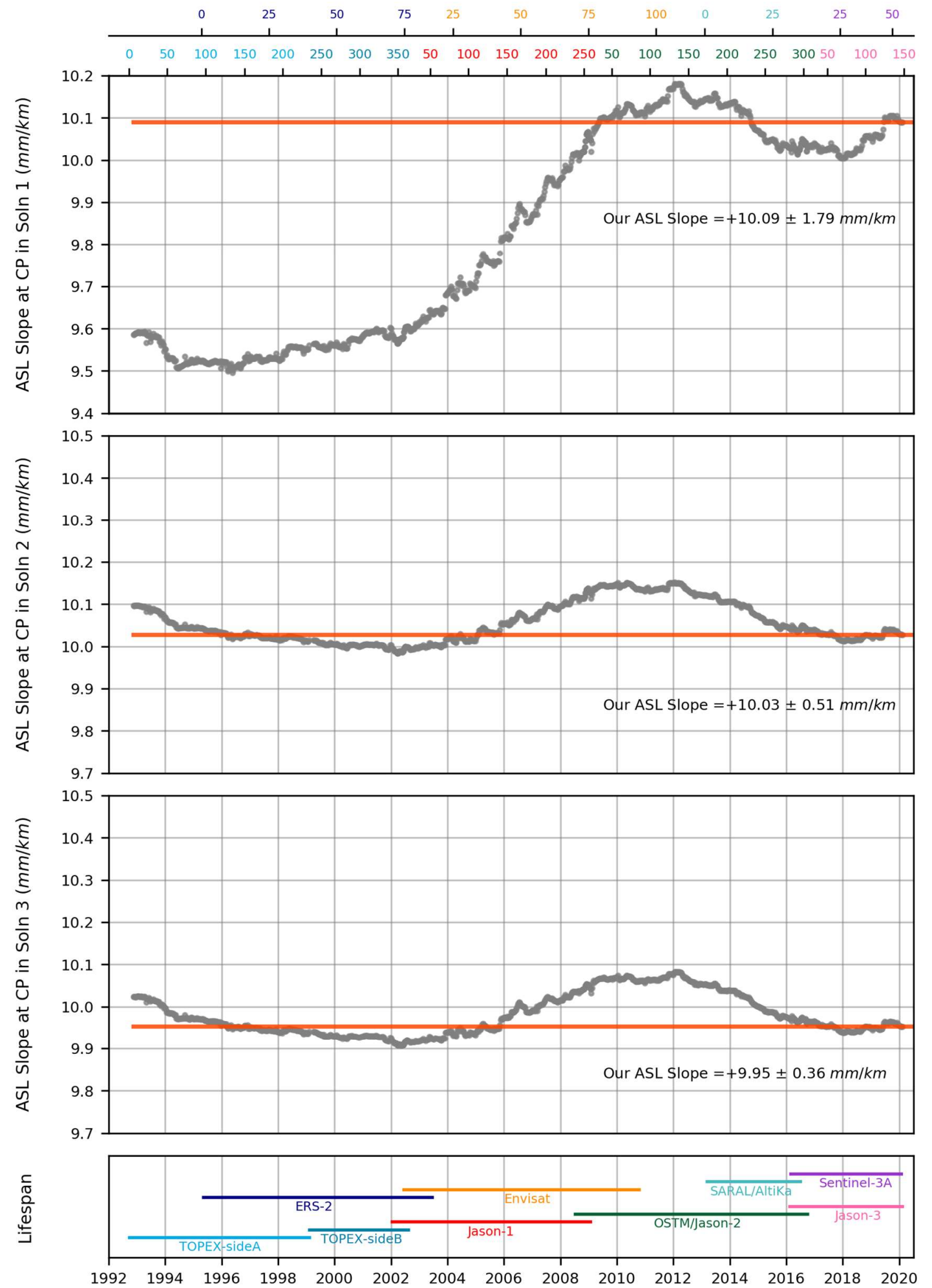

Figure S28. Cycle-by-cycle estimates of ASL slope for a representative CP in the vicinity of BURN TG in the final iterations of (top) Solution 1, (middle) Solution 2 and (bottom) Solution 3 within our multi-stage approach. The mission lifespans are illustrated at the bottom. The grey lines show the forward filtering estimates, while the orange lines show the return smoothing results. Note different scales on y-axes. 

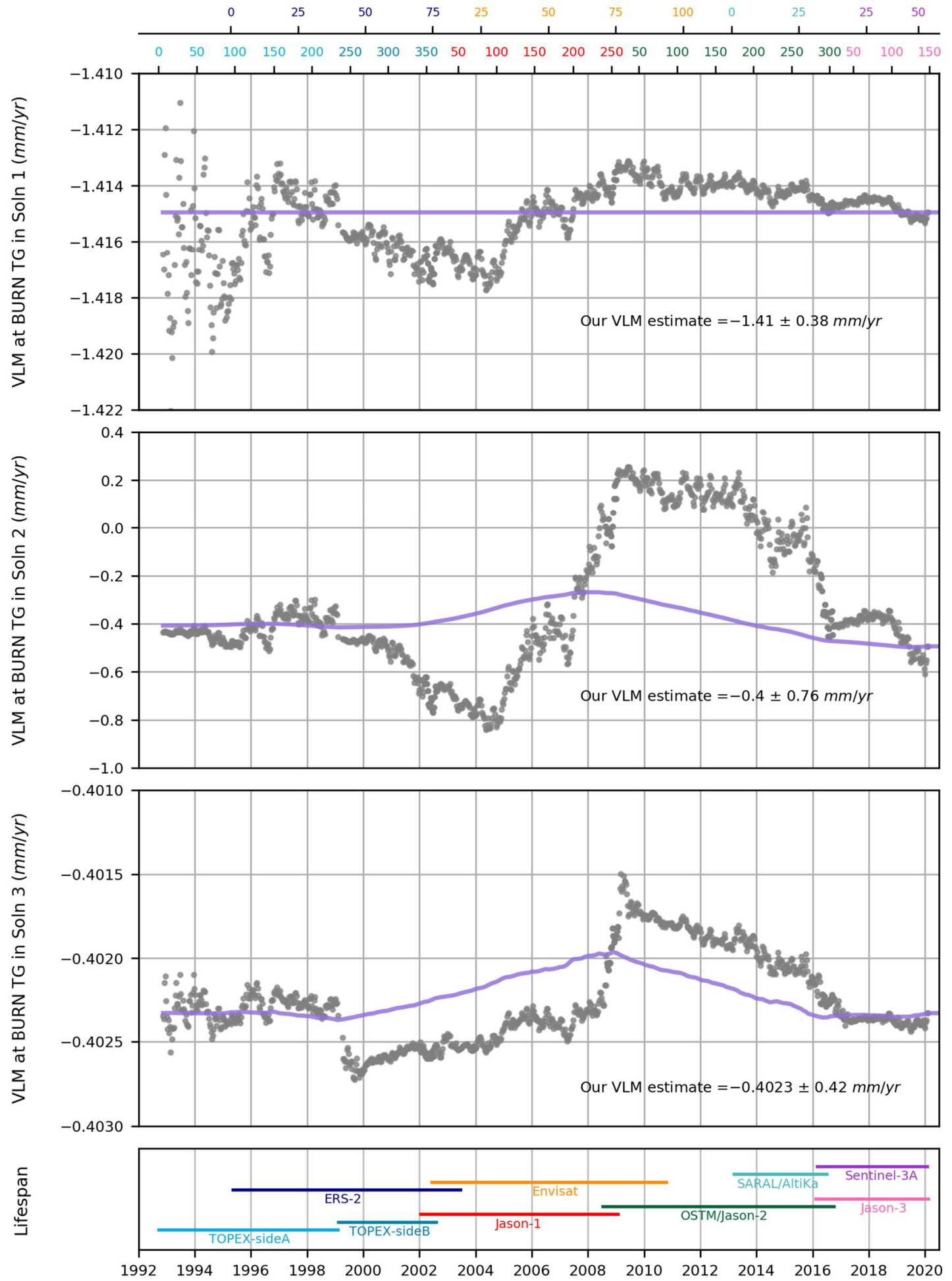

Figure S29. Cycle-by-cycle estimates of linear VLM at BURN TG in the final iterations of (top) Solution 1, (middle) Solution 2 and (bottom) Solution 3 within our multi-stage approach. The mission lifespans are illustrated at the bottom. The grey lines show the forward filtering estimates, while the purple lines show the return smoothing results. Note the very different scales on y-axes to emphasize the level of constraint imposed. The annotated values are the weighted averages of smoother estimates from each solution. 
1638

1639

1640

1641

1642

1643

1644

1645

1646

1647
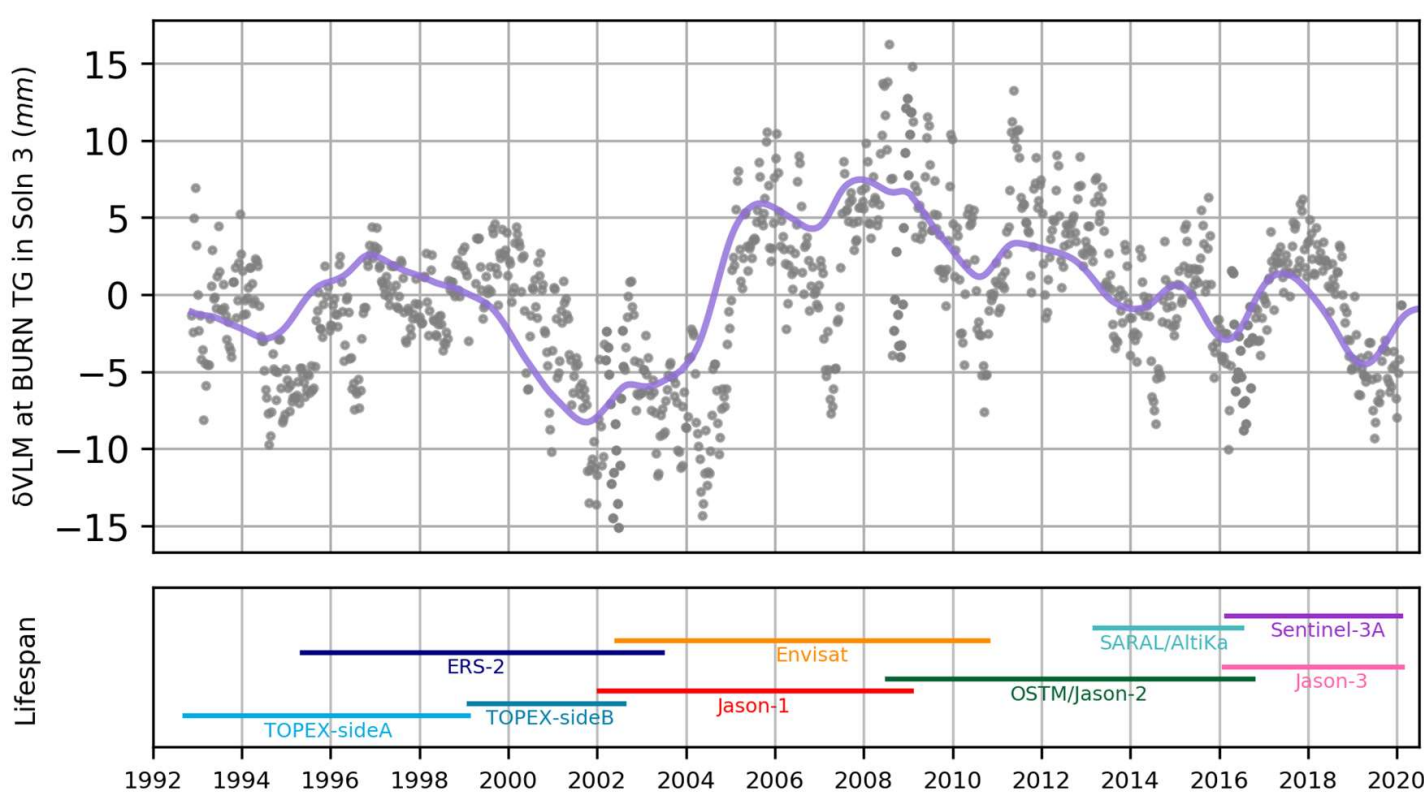

Figure S30. Cycle-by-cycle estimates of non-linear VLM at BURN TG in the final iterations of Solution 3 within our multi-stage approach. The mission-specific timespans are illustrated in the lower panel. The mission lifespans are illustrated in the lower panel. 

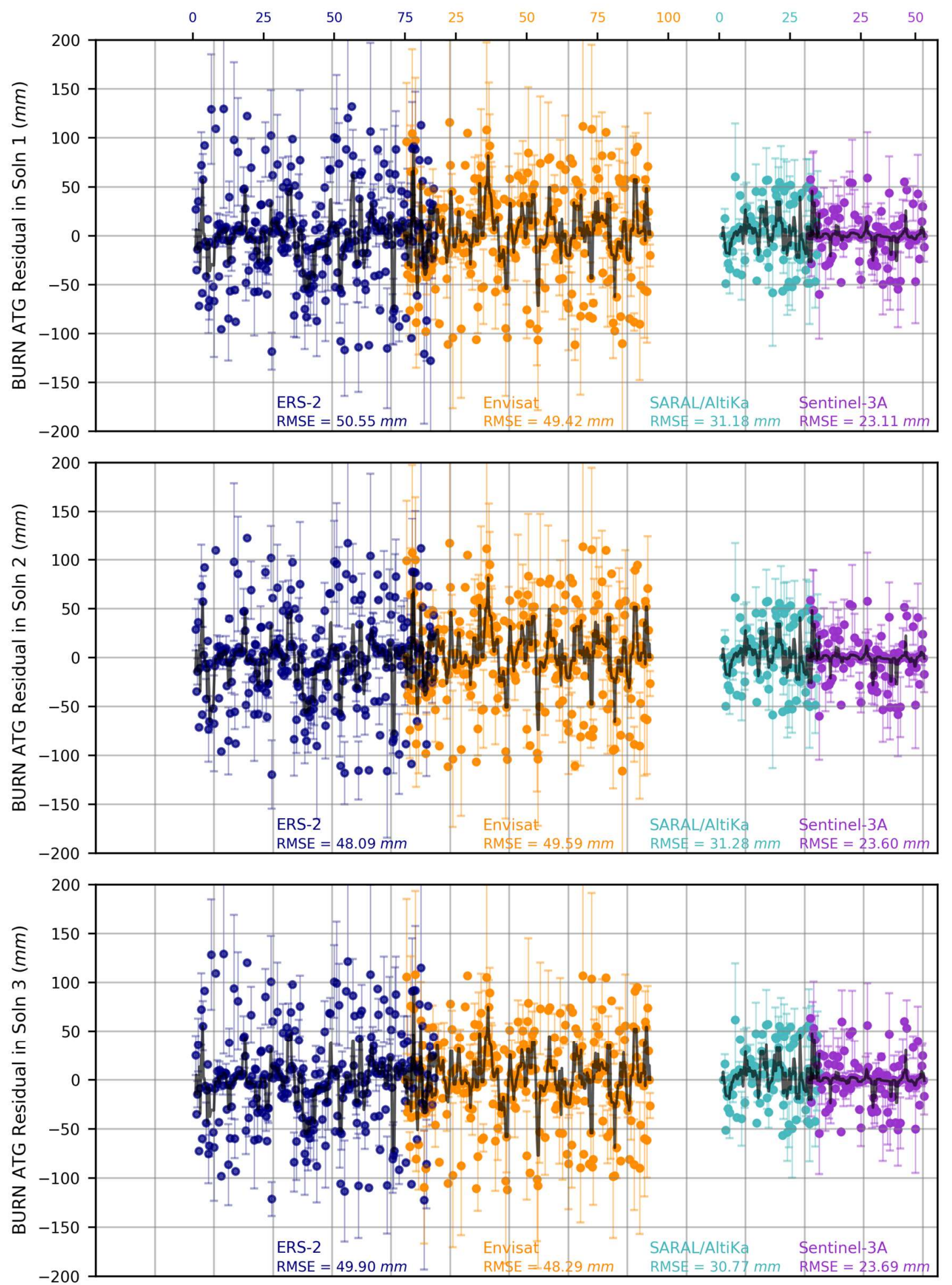

Figure S31. Cycle-by-cycle weighted average of "white plus AR1" residuals of ATG combinations specific 1651 to BURN TG in the final iterations of (top) Solution 1, (middle) Solution 2 and (bottom) Solution 3 within our multi-stage approach. 
manuscript submitted to Journal of Geodesy
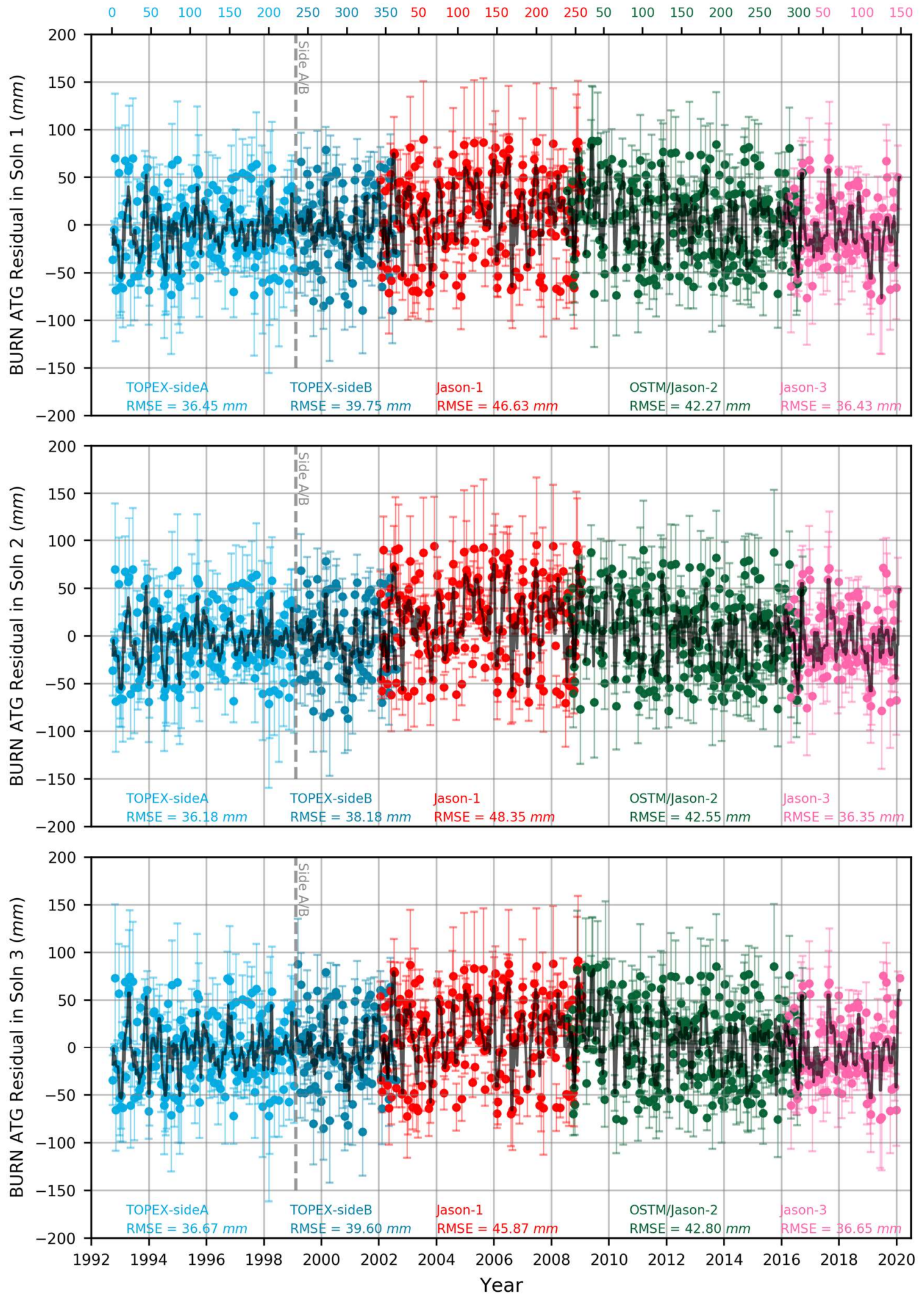

1652

1653

1654

1655

Figure S32. Same as for Figure S31, but for ATG observations associated with the reference missions. 


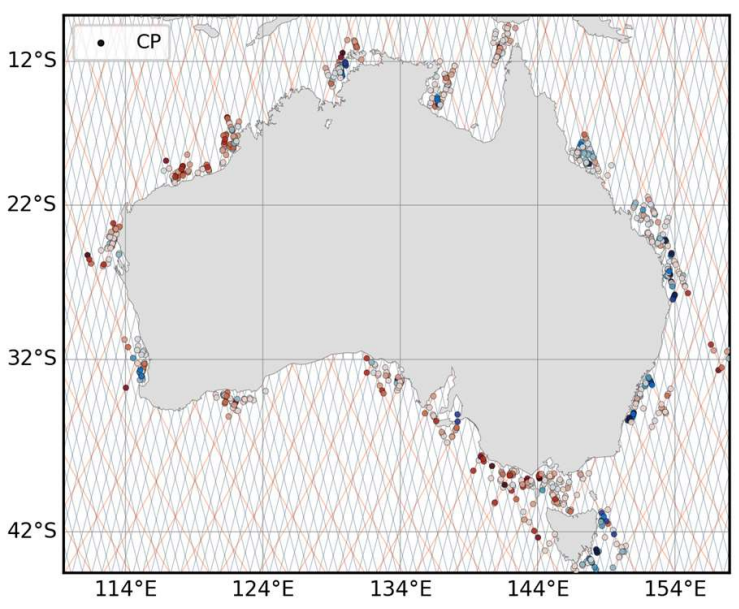

(a) Our ASL slope estimate

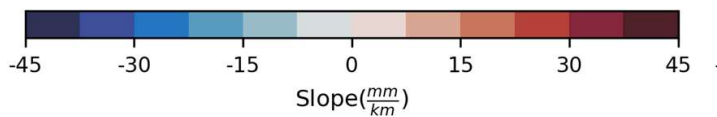

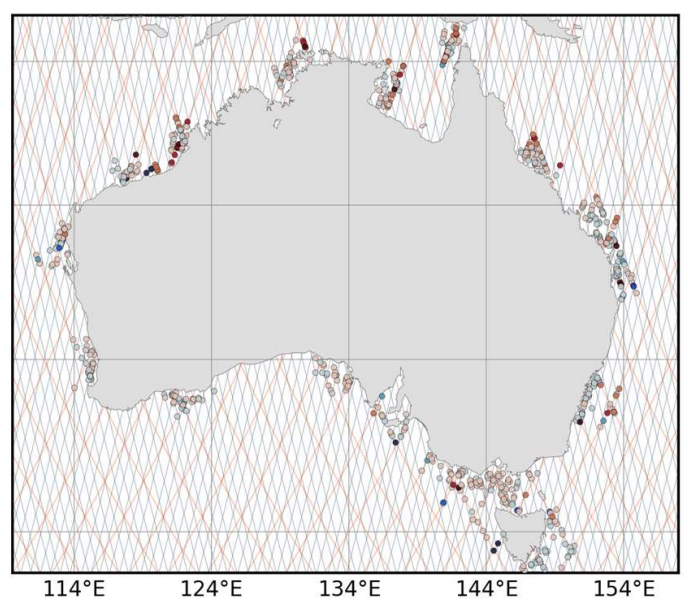

(b) ASL slope difference

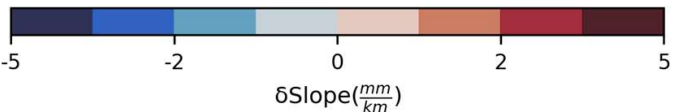

Figure S33. Map of (a) our ASL slope estimates, and (b) ASL slope differences, DTU15 mean sea surfacederived a priori values subtracted from our results. Note the negligible differences between a priori and a posteriori ASL slopes.

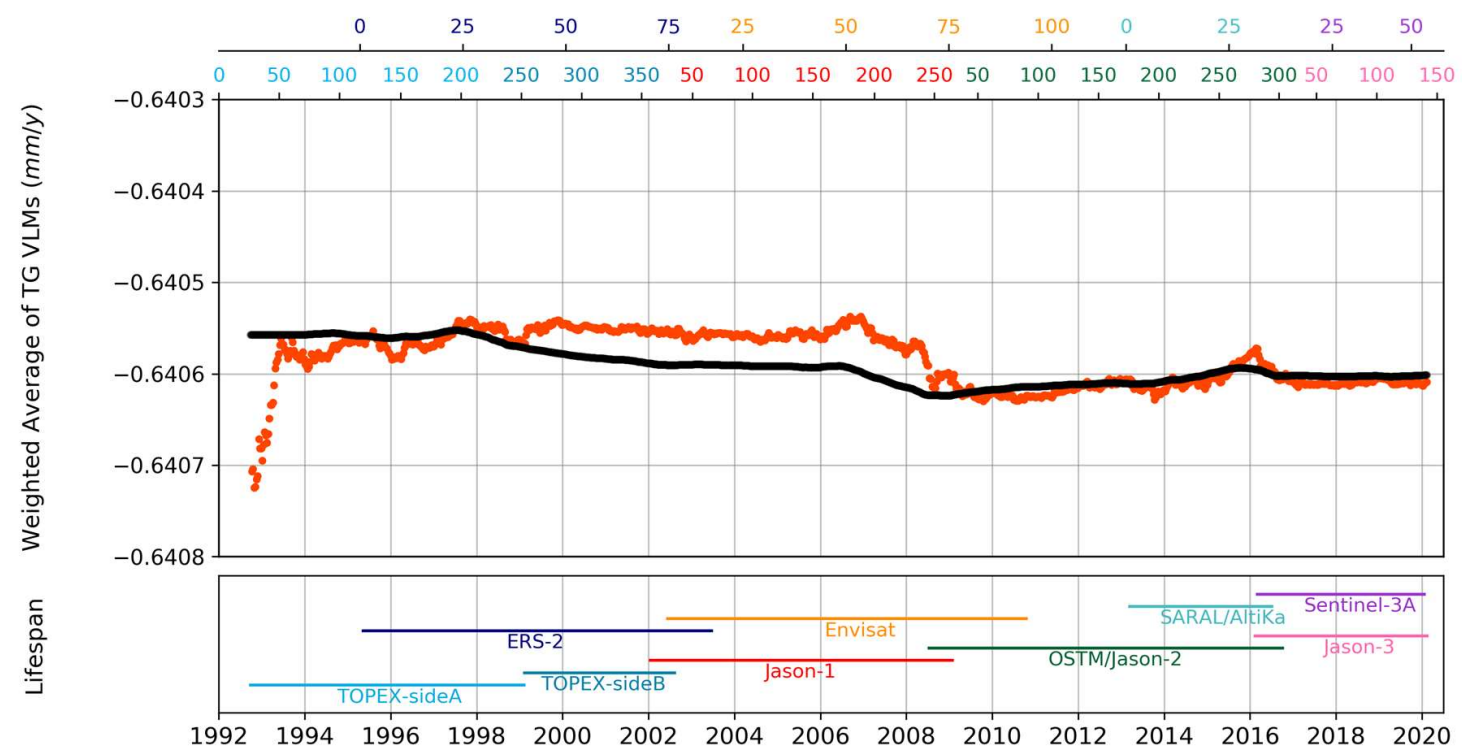

Figure S34. Cycle-by-cycle weighted average of linear TG VLM estimates from Solution 3. Note very slight change of estimates as the indication of stability of our solution datum in time. The mission-specific timespans are illustrated in the lower panel. 